Repository of the Max Delbrück Center for Molecular Medicine (MDC) Berlin (Germany)

\title{
Der1 promotes movement of misfolded proteins through the endoplasmic reticulum membrane.
}

Mehnert, M., Sommer, T., Jarosch, E. 


\title{
Der1 promotes movement of malfolded proteins through the endoplasmic reticulum
} membrane.

Martin Mehnert ${ }^{1}$, Thomas Sommer ${ }^{1,2,3}$ and Ernst Jarosch ${ }^{1,3}$

\author{
${ }^{1}$ Max-Delbrück-Center for Molecular Medicine, Robert-Rössle-Str. 10, 13125 Berlin-Buch, \\ Germany and ${ }^{2}$ Humboldt-University Berlin, Institute of Biology, Invalidenstr. 43, 10115 Berlin, \\ Germany \\ ${ }^{3}$ Correspondence should be addressed to Thomas Sommer (tsommer@mdc-berlin.de) or \\ Ernst Jarosch (ejarosch@mdc-berlin.de).
}

\begin{abstract}
Misfolded proteins of the secretory pathway are extracted from the endoplasmic reticulum $(E R)$, polyubiquitylated by a protein complex termed the $\mathrm{Hmg}-\mathrm{CoA}$ reductase degradation ligase (HRD-ligase) and degraded by cytosolic $26 \mathrm{~S}$ proteasomes. The movement of these proteins through the lipid bilayer is assumed to occur via a protein conducting channel of unknown nature. We show that the integral membrane protein Der1 oligomerises which relies on its interaction with the scaffolding protein Usa1. Mutations in the transmembrane domains of Der1 block the passage of soluble proteins across the ER-membrane. As determined by site-specific photocrosslinking the ER-luminal exposed parts of Der1 are in spatial proximity to the substrate receptor Hrd3 whereas the membrane-embedded domains reside adjacent to the ubiquitin ligase Hrd1. Intriguingly, both regions also form crosslinks to client proteins. In summary our data imply that Der1 initiates the export of aberrant polypeptides from the ER-lumen by threading such molecules into the ER-membrane and routing them to Hrd1 for ubiquitylation.
\end{abstract}

\section{Introduction}

Folding of newly synthesised proteins in the secretory pathway is monitored by a quality control system that routes terminally aberrant polypeptides from the endoplasmic reticulum $(E R)$ into the cytosol where they are polyubiquitylated and degraded by proteasomes. This process, termed ER-associated protein degradation (ERAD) 1, 2, is pivotal for the maintenance of cellular homeostasis and its mechanistic exploration will contribute to the treatment of various neurodegenerative disorders ${ }^{3-5}$ and folding diseases like cystic fibrosis 6,7. Key components of ERAD are large multi-subunit ubiquitin ligases, which are embedded in the ER-membrane ${ }^{8}$. In yeast the HRD-ligase promotes the degradation of soluble ERluminal as well as membrane-bound substrates. The core of this complex encompasses the 
RING-finger type ubiquitin ligase Hrd1, which is integrated in the ER-membrane and exposes its catalytically active centre into the cytoplasm ${ }^{9},{ }^{10}$. Hrd1 teams up with the substrate receptors Hrd3 and Yos9, which expose large soluble domains into the ER-lumen, and Usa1, which facilitates the assembly of the ligase complex ${ }^{11-15}$. Intriguingly, another subunit Der1, an integral membrane protein with several transmembrane segments that is recruited to Hrd1 via Usa1, is only required for the turnover of soluble but dispensable for the degradation of membrane-bound substrates ${ }^{16-19}$. Because Der1 weakly binds client proteins it has been speculated that it either resembles a receptor for soluble targets or partakes in the dislocation of such polypeptides from the ER ${ }^{12,20}$. How aberrant proteins are exported from the ER in the course of ERAD is unknown. Remarkably, fully glycosylated and even partially folded ERAD substrates have been shown to traverse the ER-membrane prior to their degradation ${ }^{21}$. This transport relies on the activity of the ubiquitin ligases and is generally believed to involve a proteinaceous channel ${ }^{22-24}$. Several candidates for the constitution of such a dislocation apparatus have been discussed ${ }^{25,26}$. Here we report that the transmembrane domains of Der1 constitute a functional entity that is important for ERAD. Furthermore, soluble and membrane-embedded parts of Der1 are in spatial proximity to other components of the HRD-ligase and to dislocating substrate molecules. Our data imply that Der1 establishes a functional link between the selection of aberrant proteins in the ERlumen and their ubiquitylation in the cytoplasm by promoting the insertion of such polypeptides into the ER-membrane.

\section{Results}

\section{Usa1-dependent integration of Der1 into the HRD-ligase promotes oligomerisation}

To assess possible functions of Der1 we were first interested on how this protein is integrated into the HRD-ligase protein complex. Usa1 links Der1 to Hrd1, which renders the ligase competent for the proteolysis of misfolded luminal ER proteins ${ }^{14,27}$. Sequence alignment of Der1 and its mammalian homologues, the Derlins, revealed an arrangement of conserved residues in the carboxyterminal region (GH179/180 and YY183/184; Supplementary Fig. 1a). The mutation of these residues specifically reduced the association with Usa1 as determined by co-immunoprecipitation experiments with Usa1-Myc (Fig. 1a and Supplementary table 1). Besides its linker function Usa1 also conveys the stability of Der $1^{14}$. Indeed, Der1 was degraded in a Hrd1- and Doa10-dependent manner in cells lacking Usa1 (Supplementary Fig. 1b). Accordingly, the Usa1 binding-deficient Der1 mutants were unstable (Supplementary Fig. 1c).

Der1 and other components of the HRD-ligase have been recently found to form oligomers ${ }^{14,28}$. To determine, which factors contribute to this process we monitored the interaction of 
Der1 equipped with a Myc-tag (Der1-Myc) and untagged Der1 in cells expressing both proteins and lacking individual components of the HRD-ligase. By precipitating Der1-Myc under native conditions we found that only the deletion of usa1 abrogated Der1oligomerisation (Fig. 1b). Mutations in the Usa1 interaction motif also caused the dissociation of Der1 oligomers (Fig. 1c). Importantly, Der1-Myc as well as co-expressed Der1 was stable in usa1-deleted cells, and thus a loss of oligomerisation cannot be explained by Der1 instability (Supplementary Fig. 1d). A carboxyterminally HA-tagged version of Der1, which is instable in usa1-deleted cells, was also associated with co-expressed Der1 in a Usa1dependent manner (Supplementary Fig. 1e). However, overexpression of Der1, for example by inducing a cellular stress response pathway termed the unfolded protein response (UPR) by Dithiothreitol-treatment ${ }^{29}, 30$, partially bypassed the requirement of Usa1 for oligomerisation (Fig. 1d). Therefore, Der1, similar to $\mathrm{Hrd} 1{ }^{20}$, exhibits an intrinsic propensity to form oligomers. Under unstressed growth conditions, however, efficient oligomerisation of both proteins relies on their assembly by Usa1.

\section{Mutation of conserved residues in the transmembrane domains of Der1 block the dislocation of CPY*}

Der1 is predominantly involved in the turnover of misfolded ER-luminal proteins but it is unknown, how it contributes to this process ${ }^{16,17}$. Because Der1 binds client proteins even in the absence of the substrate receptor $\mathrm{Hrd} 3$ it was proposed that it constitutes a substrate recruitment factor of the HRD-ligase 12, 20, 31 . However, the deletion of der1 already phenocopied the defect in the turnover of CPY*, a well established ER-luminal ERAD substrate, observed in hrd3- or hrd1-deleted cells and the combined deletion of der1 and hrd3 did not enhance this effect (Fig. 2a). In der1- and yos9-deleted cells we obtained a similar result (Fig. 2b). Moreover, the absence of Der1 could not be compensated by the simultaneous overexpression of $\mathrm{Hrd1}$ and $\mathrm{Hrd3}$ (Supplementary Fig. 2a). Immunoprecipitation experiments revealed that the amount of $\mathrm{CPY}^{*}$ associated with the HRD-ligase was strongly reduced in absence of $\mathrm{Hrd3}$, whereas it remained largely unchanged in der1-deleted cells (Fig. 2c, lane 11 and lane 10). Overall, these observations suggest a function of Der1 in line with rather than parallel to Hrd3/Yos9.

On the basis of sequence similarity to rhomboid proteases Greenblatt et al proposed six transmembrane segments for the mammalian homologue Derlin-1 ${ }^{32}$. By contrast predictions based on hydrophobicity analysis as well as detailed biochemical studies support a topology of Der1 that comprises only four transmembrane domains ${ }^{16}$. Nonetheless, the position of the transmembrane domains one and two is essentially the same in both models. We asked whether these segments are solely required for proper integration of Der1 into the HRD 
ligase or whether they constitute a distinct functional entity for ERAD. Sequence alignment of yeast Der1 and its homologues from different organisms revealed conserved hydrophilic amino acids particularly in the first and second transmembrane domains (Supplementary Fig. 1a). We changed some of these residues to alanine or leucine and monitored the effects on ERAD (Supplementary table 1). Mutations in the first transmembrane segment (R17L and C23L; Der1RC) moderately affected the turnover of the prevalent ERAD substrate CPY* (Fig. 2d). A stronger defect was observed for the exchange of a conserved asparagine residue (N73L; Der1N) in the second transmembrane domain. The combination of R17L with N73L (Der1RN) caused a severe delay in $\mathrm{CPY}^{*}$-degradation. Notably, these mutations also stalled the turnover of $\mathrm{PrA}^{*}$, another well established soluble luminal ERAD substrate, whereas the degradation of 6xMyc-Hmg2, which relies on the HRD-ligase but does not require Der1 ${ }^{33}$, proceeded at similar rates in wild-type and Der1 mutant cells (Supplementary Fig. 2b). Mutations in the first luminal loop or in other regions of Der1 did not derogate ERAD (Supplementary Table 1). Der1N, Der1RN and Der1RC are stably expressed at levels comparable to the wild-type protein (Supplementary Fig. 2c). Moreover, as determined by immunoprecipitation the ability to form oligomers and the association with components of the HRD-ligase were not affected (Supplementary Fig. 2d and 2e).

In cells deleted for der1 or expressing Der1RN we were unable to detect significant amounts of ubiquitylated $\mathrm{CPY}^{*}\left(\mathrm{CPY}^{*}-\mathrm{Myc}-\mathrm{Ub}\right.$; Fig. 2e, lanes 10, 12). Because protein ubiquitylation is exclusively catalysed in the cytoplasm, the export of substrates from the ER appeared to be disturbed. Indeed, we found that most of $\mathrm{CPY}^{*}$ was protected from Proteinase $\mathrm{K}$ treatment in lysates of these cells indicative for a localisation within a membrane bound compartment (Fig. 2f). By contrast, Der1RC cells contained low amounts of ubiquitylated CPY* and displayed a partial sensitivity of $\mathrm{CPY}^{*}$ to Proteinase $\mathrm{K}$ corresponding to the milder degradation defect in this mutant (Fig. 2e, lane 11; Fig. 2f).

\section{Spatial proximity of Der1 to components of the HRD-ligase}

To get further insights in the function of the Der1 transmembrane domains we employed an in vivo site-specific photocrosslinking approach ${ }^{34,35}$. Der1-Myc was labelled in living cells with the photoreactive amino acid analogue p-Benzoylphenylalanine (pBpa) by overexpressing plasmid-encoded constructs that harbour a single amber stop codon at defined positions. The expression of a suppressor tRNA charged with pBpa allowed the incorporation of this crosslinker at the specified locations. Upon irradiation of cells with ultra violet (UV) light pBpa was activated and crosslinked to neighbouring proteins. Der1-Myc was then precipitated with anti-Myc antibodies from cell lysates and the samples were analysed by immunoblotting. We focussed our studies on positions in the transmembrane domains as 
well as the first luminal loop of Der1 because these parts appeared to be most conserved. Notably, the overexpression of Der1 does not significantly interfere with the degradation of luminal substrates (Supplementary Fig. 3c) ${ }^{14,20}$. Furthermore, we conducted most of these experiments in cells expressing a catalytically inactive variant of the ERAD-specific ubiquitin conjugating enzyme Ubc7 (Ubc7C/S) to abrogate ERAD and thereby adjust the cellular levels of substrate molecules.

We found prominent crosslinking products with Hrd1 at several positions in the aminoterminal part of the first and less intense crosslinks at few positions in the second transmembrane region of Der1 (Fig. 3; positions V25, S27, L29, S31, R33 and V35; F66, W68, I72 and F75). In cells lacking the Der1 adaptor Usa1 we were unable to detect such crosslinks demonstrating the specificity of the reaction (Fig. 4a). Crosslinks to Usa1 were almost exclusively observed for positions in the region around amino acids 146 to 152 , which is consistent with the notion that the downstream part of Der1 is required for the binding to Usa1 (Fig. 3; positions I146, V148, P150 and 1152). Surprisingly, we also detected major crosslinks to the putative substrate receptor Hrd3 predominantly for residues in the first ERluminal loop of Der1 (Fig. 3; positions G38, L46, K50 and Q52) whereas reactions to the Hrd3-associated lectin Yos9 were not found (Supplementary Fig. 3a). Again, the crosslinking reactions depended on the recruitment of Der1 to the HRD-ligase via Usa1 (Fig. 4b). A functional variant of $\mathrm{Hrd} 3$ that is deleted for the transmembrane region and only comprises the luminal domain (Hrd3 1-769) ${ }^{12}$ also formed crosslinks with the pBpa-labelled Der1 variants demonstrating a close spatial proximity between the luminal parts of both proteins (Fig. 4c). Some of the Der1-Hrd3 crosslinks involved residues that were calculated to map to the integral membrane domains of Der1 (Fig. 3; positions R33 and D62). Although we cannot formally rule out that the position of the transmembrane segments slightly differs from their predicted location, this indicated that the Hrd3 luminal domain is capable to immerge into the membrane-embedded part of Der1. By co-expressing pBpa-labelled Der1-Myc and unlabelled Der1-HA we detected crosslinking products of these proteins primarily for positions in the transmembrane regions (Fig. 4d). This finding confirms oligomer-formation of Der1 as suggested by the immunoprecipitation experiments and implies a tight association of the membrane-embedded segments within this assembly.

Strikingly, pBpa-labelled Der1 could also be crosslinked to the ERAD substrate CPY*-HA. The most prominent crosslinks involved the luminal loop one and the luminally orientated parts of the Der1 transmembrane segments one, two and four (Fig. 3 and Supplementary Fig. 3b, e.g. positions R33, G38, K50, 160, D62 and I146). Position G38, which maps to the first ER-luminal loop of Der1, was of special interest because this residue appeared to be localised in close proximity to substrate as well as to the substrate receptor Hrd3. Unlabelled Der1-Myc did not form crosslinks with $\mathrm{CPY}^{*}-\mathrm{HA}$ or components of the HRD-ligase (Fig. 3; 
outmost left lanes). Moreover, no crosslinks were detected when the UV-treatment was omitted. The modification of Der1 with pBpa did not affect the stability of the protein and had no significant effect on the activity of Der1 (Supplementary Fig. 3c). Furthermore, pBpalabelled Der1 variants were properly assembled into the HRD ligase as determined by the immunoprecipitation of selected constructs (Supplementary Fig. 3d). These results identified Der1 as a central factor in the HRD-ligase protein complex and implied that ER-luminal as well as membrane-embedded regions of Der1 reside in close contact to a soluble degradation substrate.

\section{Der1 directly binds substrate molecules}

Previous work indicated that Der1 exhibits a weak but specific substrate binding activity ${ }^{12}$. We therefore wanted to determine the impact of the substrate receptor Hrd3 on the Der1$\mathrm{CPY}^{*}$ crosslinks. To level out cellular protein amounts of $\mathrm{CPY}^{*}-\mathrm{HA}$ we conducted these experiments in Ubc7C/S cells (see above). The amount of $\mathrm{CPY}^{*}$ that crosslinked to specific positions in the luminal loop of Der1 was substantially increased in the hrd3-deleted strain, which correlates to previous studies ${ }^{31}$ (Fig. 5a). Likewise, the release of Der1 from the HRDligase in cells lacking Usa1 mildly affected substrate crosslinking at positions in the luminal loop (Supplementary Fig. 4a). Notably, due to the overexpression of Der1 in the crosslinking setup the oligomeric state of the protein is still intact despite the deletion of usa1 (Supplementary Fig. 4b, compare lanes 5 and 6). Consistently, substrate binding to Der1Myc or Der1-HA neither depended on Usa1 nor on $\mathrm{Hrd3}$ as determined by immunoprecipitation in Ubc7C/S cells (Fig. 5b, compare lanes 6, 7 and 8; Supplementary Fig. 4c, compare lanes 5 and 6). To substantiate the specificity of the Der1-CPY* crosslinks we compared wild-type and ubiquitylation-deficient Ubc7C/S cells in the crosslinking experiments. The overall crosslinking pattern to Hrd1, Usa1 or Hrd3, which form a stable complex with Der1, was not affected by the activity of the ligase (Fig. 5c, Supplementary Fig. $4 \mathrm{~d}$ and $4 \mathrm{e}$ ). However, the amount of crosslinking products was increased in Ubc7C/S cells possibly due to up-regulation of the HRD-ligase components by the UPR ${ }^{30}$ (Supplementary Fig. 4f). In wild-type cells the amount of crosslinked CPY*-HA was clearly reduced albeit the relative intensity was almost identical for selected Der1 positions. This observation matched the idea that in the course of substrate processing no prevalent degradation intermediate of $\mathrm{CPY}^{*}-\mathrm{HA}$ accumulated at Der1. Intriguingly, this pattern was changed in the $\mathrm{Ubc} 7 \mathrm{C} / \mathrm{S}$ cells indicating that substrate molecules arrested in a particular constitution at the HRD-ligase (Fig. 5c). We also observed crosslinks of the ERAD substrate PrA*-HA to defined positions of Der1. The pattern of these crosslinks correlated with those observed for $C P Y^{*}-H A$ suggesting that both substrates arrested in the same fashion at Der1 (Fig. 5d). 
Importantly, pBpa-Der1-Myc could not be crosslinked to properly folded CPY-HA (Fig. 5e) or stable ER resident proteins like Kar2, an Hsp70-type ER-luminal chaperone ${ }^{36}$, or Sec61, a membrane-bound component of the protein import apparatus ${ }^{37}$ (Supplementary Fig. $4 \mathrm{~g}$ ) demonstrating that only proteins that are targeted for degradation by the HRD-ligase come into spatial proximity to Der1.

\section{Mutations in the Der1 transmembrane domains affect the crosslinking to HRD-ligase components and CPY*-3xHA}

In the following we employed the ERAD-deficient Der1RN mutant in the crosslinking experiments. Although this mutant protein appeared to be properly integrated into the HRDligase as determined by immunoprecipitation, the amount of most crosslinking products with Hrd1, Usa1 and Hrd3 was significantly reduced indicating moderate structural changes in Der1 (Fig. 6a,b and Supplementary Fig. 5a). By contrast, the crosslinking products with CPY*-HA were increased predominantly at positions in the luminal loop of Der1 (Fig. 6a and $6 \mathrm{~b}$, right panel, compare lanes 5 and 7 ). Noteworthy, the cellular level of this substrate remained unchanged (Supplementary Fig. 5b). Moreover, the individual pBpa-labelled Der1 variants precipitated equal amounts of $\mathrm{CPY}^{*}-\mathrm{HA}$, indicating that their ability to bind substrate molecules was not affected (Supplementary Fig. 5b). This strongly implied that ERAD substrates were unable to enter a dislocation complex in Der1RN cells, which in turn caused their accumulation at the luminal-exposed parts. Importantly, the crosslinking pattern of Der1 and Der1RN to $\mathrm{CPY}^{*}-\mathrm{HA}$ was almost identical upon the release of these proteins from the HRD-ligase in cells deleted for usa1 (Fig. 6c, compare lanes 5 and 7 with Fig. 6a, lanes 5 and 7). Thus, the accumulation of a particular degradation intermediate of CPY*-HA depended on the correct integration of Der1 into the HRD-ligase.

\section{Discussion}

Prior to their degradation misfolded proteins of the secretory pathway are routed from the ER into the cytoplasm. Intriguingly, these proteins are often modified by complex glycans, contain disulfide bridges and may be partially folded. This poses special requirements for the movement of such polypeptides through the ER-membrane. Previous work indicated that mammalian Derlin-1, a homologue of Der1, is involved in this process ${ }^{38,39}$. When overexpressed in mammalian cells, Derlin-1 and also the closely related Derlin-2 and -3 proteins were shown to form oligomers, which are associated with ERAD ligases ${ }^{40-42}$. Moreover, a protein complex containing Derlin-1 bound to client proteins before and after their extraction from the ER ${ }^{38}$. Concordantly, the release of a model substrate from microsomes in a reconstituted system was efficiently inhibited by the addition of Derlin-1- 
specific antibodies ${ }^{43}$. Although similar in size and topology Der1 and the Derlin proteins exhibit functional differences. The Derlins share certain amino acid motifs with inactive rhomboid proteases and contain a so-called SHP-box motif in their cytoplasmic carboxyterminal domain for direct binding to the AAA-ATPase p97, which are less conserved or not present in Der1 ${ }^{32}$. Moreover, the mammalian Derlin proteins are involved in the turnover of soluble and membrane-bound proteins, while Der1 is almost exclusively required for the degradation of soluble, ER-luminal substrates ${ }^{38,44,45}$.

Our data now provide strong evidence that yeast Der1 directly partakes in the extraction of aberrant proteins from the ER-lumen. First, upon Usa1-dependent assembly into the HRDligase Der1 forms oligomers in the ER-membrane, which may be a prerequisite to enable the transit of substrate-proteins across a lipid layer. Second, the exchange of conserved polar residues in the transmembrane domains of Der1 abolishes export of polypeptides from the ER without affecting the overall topology of the HRD-ligase. Third, client proteins form crosslinks to ER-luminal as well as membrane-embedded parts of Der1 indicating that they are in close proximity to Der1 during dislocation from the ER. We are aware that the in vivo crosslinking approach allows the unspecific incorporation of $\mathrm{pBpa}$ into proteins that are naturally terminated by an amber stop-codon, which causes the production of abnormal species with carboxyterminal extensions. Still, we did not observe physiological alterations in pBpa-labelled cells and were unable to detect crosslinking products to unlabelled Der1-Myc. Fourth, the efficiency of pBpa-Der1 crosslinking to substrate molecules depends on the activity of the ligase and the assembly of Der1 into the ligase complex. And fifth, Der1 is located in the immediate vicinity of the substrate receptor $\mathrm{Hrd} 3$ as well as the ubiquitin ligase Hrd1 and is therefore at a prominent position to functionally link substrate selection in the ER-lumen with the ubiquitylating activity at the cytoplasmic face of the ER.

Based on these observations we propose the following model for Der1 activity (Fig. 7). Malfolded proteins engage $\mathrm{Hrd} 3$ and Yos9 where they are selected for degradation. Those receptors then pass substrates to Der1, which acts downstream. Der1 contains regions in the ER-lumen that are in close proximity to $\mathrm{Hrd} 3$ and weakly bind aberrant polypeptides. This activity does not bypass the requirement for Hrd3 and Yos9 in ERAD. Rather, the access to Der1 appears to be restricted to client molecules that were pre-selected by Hrd3/Yos9. In the absence of Hrd3 Der1 probably binds polypeptides with low affinity, which does not suffice to trigger their degradation. Interestingly, mutations in the ER-luminal part of mammalian Derlin1 abolished its function in ERAD ${ }^{32}$. We did not observe a similar effect for the corresponding positions in Der1 but we did not systematically investigate this issue. Next, Der1 initiates the insertion of substrate molecules into the ER-membrane. The assembly of multiple Der1 subunits may generate a flexible funnel that supports immersion of even folded client proteins. Importantly, this step guides the substrates in spatial proximity to Hrd1 and 
arranges them for ubiquitylation. The subsequent movement through the membrane may involve a proteinacious pore that contains Der1 and other proteins. Recently, the ubiquitin ligase Hrd1 has been implicated in the formation of such a conduit ${ }^{14,20}$. Hrd1 contains six transmembrane segments and also forms oligomers. While a mild 5-10 fold overexpression of Hrd1 failed to compensate for a loss of Der1 (Supplementary Figure 2a), strong overexpression of this protein partially substituted the function of Der1, Usa1, Hrd3 or Yos9 20. Moreover, arrested dislocation intermediates could be crosslinked to Hrd1, which depended on the catalytic activity of the HRD-ligase ${ }^{20}$. By contrast, crosslinks of substrates with Der1 were increased in a ubiquitylation deficient mutant. Since ubiquitylation was found to be mandatory for protein extraction from the ER this modification most likely prevents the dissociation of client proteins from Hrd1 and drives their export via the ubiquitin-dependent AAA-ATPase Cdc48/p97 ${ }^{46}$. Still, mutations in the Hrd1 transmembrane segments, which blocked the degradation of membrane-bound targets, had no impact on the turnover of soluble client proteins ${ }^{47}$. Hence, further studies are needed to elucidate the detailed composition of the conduit that channels soluble ERAD substrates through the ERmembrane.

\section{Acknowledgements}

The authors wish to thank the members of the laboratory for helpful and stimulating discussions, unpublished data, and materials. We thank Corinna Volkwein for excellent technical assistance. We would like to thank P.G. Schultz for materials needed for the in vivo cross-linking experiments. T.A. Rapoport, D.H. Wolf and R.Y. Hampton are acknowledged for providing antibodies and plasmids. The Deutsche Forschungsgemeinschaft generously supports TS and EJ (JA 1830/1-2, SFB 740, Priority Program 1365, and the German-Israel Project Cooperation DIP).

\section{Author contributions}

M.M. performed all experiments except Supplementary Fig. 2a and Supplementary Fig. 3c (E.J.). The experiments were designed by M.M. and E.J.. T.S. guided the project planning. M.M. and E.J. wrote the manuscript.

\section{Competing financial interests}

The authors declare that they have no competing financial interests. 


\section{Figure legends}

\section{Figure 1}

The carboxyterminus of Der1 is required for oligomerisation and integration into the HRDligase.

a, Total cell extracts from Usa1-Myc $\Delta d e r 1 \Delta u b c 7$ cells expressing various plasmid-encoded Der1 point mutants were solubilised with Digitonin and subjected to immunoprecipitation with antibodies against the Myc-epitope. The precipitates were analysed by SDS-PAGE and immunoblotting using specific antibodies. b, Der1-Myc was precipitated from cell lysates of yeast strains deleted for the given genes under non-denaturing conditions and the samples were analysed as described above. c, Yeast cells expressing Der1-Myc were transformed with plasmids encoding the given Der1 mutants. Der1-Myc was precipitated and the samples analysed as above. The asterisk denotes unspecific reactivity with the heavy chains of the antibody used in the immunoprecipitation. d, Yeast cells expressing Der1 and Der1-Myc were treated with $2 \mathrm{mM}$ Dithiothreitol (DTT) for 2 hours to induce the unfolded protein response. Der1-Myc was then precipitated from cell lysates prepared under non-denaturing conditions and the samples were analysed as above. The asterisk denotes unspecific reactivity of the antibody as in $\mathbf{c}$.

\section{Figure 2}

Mutations in the transmembrane domains of Der1 abolish the dislocation of luminal ERAD substrates.

$\mathbf{a}, \mathbf{b}$, Pulse chase experiment to monitor $\mathrm{CPY}^{*}$ turnover in the indicated yeast strains. Error bars represent standard deviation of three independent experiments. c. Microsomes were prepared from cells of the indicated genotype and solubilised with NP40. HA-tagged Hrd1 was immunoprecipitated with anti-HA antibodies and the bound proteins were analysed by SDS-PAGE and immunoblotting. d, Graphical presentation of three independent experiments monitoring $\mathrm{CPY}^{*}$ degradation in the indicated Der1 mutant strains by pulse chase analysis as in a. e, CPY*-Myc was immunoprecipitated from total cell lysates with anti-Myc antibodies. The precipitates were analysed by SDS-PAGE and immunoblotting using anti-ubiquitin antibodies. Rpt4R refers to a yeast strain expressing a dysfunctional version of the proteasomal AAA-ATPase Rpt4, which causes the accumulation of ubiquitylated proteins. The asterisk denotes unspecific reactivity with the heavy chains of the antibody used in the immunoprecipitation. f, Protease-protection assay to monitor the export of $\mathrm{CPY}^{*}$ from the ER. Cells of the indicated genotypes were incubated with cycloheximide for 1 hour and lysed under conditions that allowed the formation of vesicles (see Methods). The samples were 
treated with Proteinase $\mathrm{K}$ and Triton-X 100 or left untreated. Proteins were precipitated and analysed by SDS-PAGE. Immunoblotting with anti-Kar2 antibodies serves as control for the integrity of the vesicles. The processing of the ER luminal protein Kar2 to a proteaseresistant fragment $\left(^{*}\right)$ in absence of Triton-X 100 indicates a leakage of some vesicles.

\section{Figure 3}

pBpa-labelled Der1 forms crosslinks with components of the HRD-ligase.

Yeast cells deleted for der1 and expressing HA-tagged $\mathrm{CPY}^{*}$ as well as catalytically inactive Ubc7 ( $\triangle$ der1 CPY*-HA Ubc7C/S) were transformed with high-copy plasmids encoding Der1Myc variants controlled by the CUP promoter and containing the photoreactive amino acid analogue $\mathrm{pBpa}$ at the indicated positions (derived from pMM075). To determine the specificity of the crosslinking reaction the experiment was performed with an unmodified version of Der1-Myc (wt) (pMM075). After UV irradiation the cells were lysed and Der1-Myc was immunoprecipitated. Proteins crosslinked to pBpa-labelled Der1 were detected by immunoblotting with specific antibodies. The relative position of the Der1 transmembrane segments according to Hitt et al. ${ }^{16}$ is given by green bars (see also Supplementary Figure 1a).

\section{Figure 4}

The pBpa-labelled Der1 variants are properly integrated into the HRD-ligase.

a, Der1-Myc constructs (derived from pMM075) containing photoreactive probes at positions which show prominent crosslinks with Hrd1 were expressed in $\Delta d e r 1 \mathrm{Ubc7C/S} \mathrm{CPY}{ }^{*}-\mathrm{HA}$ cells lacking Usa1 where indicated and subjected to in vivo photocrosslinking as described (see Methods). The asterisks mark signals that cross-react with the anti-Myc antibody. $\mathbf{b}$, As in a but the photoreactive probes were placed at positions which were found to display intense crosslinks with Hrd3.c, As in abut the labelled Der1-Myc constructs were transformed into $\Delta d e r 1$ cells that either express full length Hrd3 or the luminal domain of Hrd3 (1-767).d, $\Delta d e r 1 \mathrm{Ubc7C/S}$ cells were transformed with pBpa-labelled Der1-Myc constructs (derived from pMM075) and a high-copy number plasmid encoding Der1-HA (pMM079). Upon UV irradiation and cell lysis Der1-Myc was precipitated and crosslinks to Der1-HA were detected by anti-HA antibodies via immunoblotting.

\section{Figure 5}

pBpa-Der1 crosslinks to luminal ERAD substrates.

a, Der1-Myc labelled with pBpa at positions in the first luminal loop (G38, Y42) and in the second transmembrane region (S70) (derived from pMM075) were expressed in $\Delta d e r 1$ CPY*-HA Ubc7C/S cells that lacked Hrd3 where indicated. After UV irradiation Der1-Myc 
was immunoprecipitated from cell lysates and analysed as in Figure 3. The asterisks mark signals that cross-react with the antibodies. b. Microsomes from cells of the indicated genotype were solubilized with NP-40 and Der1-Myc was precipitated with anti-Myc antibodies. To adjust the cellular levels of substrate molecules the experiment was performed in cells deleted for $u b c 7$ (lanes 4 and 8 ) or expressing a catalytically inactive mutant of this enzyme (Ubc7C/S) (lanes 1-3 and 5-7). c, As in a but Der1-Myc was either expressed in $\Delta d e r 1 \mathrm{CPY}^{*}$-HA cells (wt) or in $\Delta d e r 1 \mathrm{CPY}^{*}$-HA Ubc7C/S cells. d, $\Delta u b c 7$ cells expressing either properly folded CPY-HA (wt) or the misfolded variant (mut) were transformed with Der1-Myc constructs that incorporated the photoreactive crosslinker pBpa at positions in the first luminal loop (G38) and in the second transmembrane domain (D62, S70) (derived from pMM075). The crosslinking experiment was performed as in a. The signal intensity of CPY-HA in the input lanes was reduced compared to CPY*-HA due to vacuolar processing of the HA-epitope. e, Der1-Myc labelled with pBpa at positions in the first (R33), second (D62, F66, S70) or fourth (I146) transmembrane domain or in the first luminal loop (G38) (derived from pMM075) were expressed in Ubc7C/S cells that lacked endogenous Proteinase A ( $\Delta$ pep4) and contained a low-copy plasmid encoding PrA*-HA (pMM076). By using an unlabelled version of Der1-Myc (pMM075) the specificity of the crosslinking reaction was determined. The crosslinking experiment was performed as in a.

\section{Figure 6}

Dislocation deficient point mutants of Der1 affect the crosslinking to HRD-ligase components and $\mathrm{CPY}^{*}-\mathrm{HA}$.

a, $\triangle$ der1 Ubc7C/S CPY*-HA cells were transformed with high-copy plasmids encoding either Der1-Myc (derived from pMM075) or Der1RN-Myc (derived from pMM074) which contain photoreactive probes in the first (S31) and fourth (I146) transmembrane domain or in the first luminal loop (G38). Upon UV irradiation and cell lysis, the Der1-Myc variants were subjected to immunoprecipitation with anti-Myc antibodies. The crosslinked interaction partners were analysed by SDS-PAGE and immunoblotting using specific antibodies. b, Positions which show significant crosslinks with Hrd1 (left panel) or Hrd3 (right panel) were modified with pBpa in Der1-Myc and Der1RN-Myc, respectively. The Der1 constructs were expressed in $\Delta d e r 1$ Ubc7C/S CPY*-HA cells and pBpa was activated by UV light. c, As in a but the Der1Myc variants were expressed in $\Delta d e r 1 \mathrm{Ubc7C/S} \mathrm{CPY}^{*}-\mathrm{HA}$ cells that were deleted for usa1.

\section{Figure 7}

A model for the function of Der1 in the dislocation of ERAD substrates.

Misfolded ER-luminal proteins (red) are bound by the receptors Hrd3 and Yos9 (blue) in the ER-lumen. Selected substrate molecules are then transferred to Der1 (green). Der1 weakly 
binds aberrant polypeptides via its ER-luminal exposed parts that are shielded by Hrd3 in the assembled ligase complex. The association with Der1 initiates the insertion of the substrates into the ER-membrane. Notably, the transmembrane domains of Der1 appear to be important for this process. Subsequently the substrate molecules are routed to the ubiquitin ligase Hrd1 (orange) for ubiquitylation. This modification stabilises the association of substrates with the HRD-ligase and triggers their dislocation from the ER. Complete movement of client proteins through the ER-membrane most likely occurs via a conduit containing the transmembrane segments of Der1 and Hrd1 and depends on the activity of the ubiquitin-specific AAAATPase Cdc48 (not shown).

\section{References}

1. Hirsch, C., Gauss, R., Horn, S.C., Neuber, O. \& Sommer, T. The ubiquitylation machinery of the endoplasmic reticulum. Nature 458, 453-460 (2009).

2. Brodsky, J.L. Cleaning up: ER-associated degradation to the rescue. Cell 151, 11631167 (2012).

3. Kaneko, M. et al. Loss of HRD1-mediated protein degradation causes amyloid precursor protein accumulation and amyloid-beta generation. J Neurosci 30, 39243932 (2010).

4. Maeda, T. et al. An E3 ubiquitin ligase, Synoviolin, is involved in the degradation of immature nicastrin, and regulates the production of amyloid beta-protein. FEBS $J$ 276, 5832-5840 (2009).

5. Yang, $\mathrm{H}$. et al. Huntingtin interacts with the cue domain of gp78 and inhibits gp78 binding to ubiquitin and p97/VCP. PLoS One 5, e8905 (2010).

6. Gelman, M.S. \& Kopito, R.R. Cystic fibrosis: premature degradation of mutant proteins as a molecular disease mechanism. Methods Mol Biol 232, 27-37 (2003).

7. Lukacs, G.L. \& Verkman, A.S. CFTR: folding, misfolding and correcting the DeltaF508 conformational defect. Trends Mol Med 18, 81-91 (2012).

8. Mehnert, M., Sommer, T. \& Jarosch, E. ERAD ubiquitin ligases: multifunctional tools for protein quality control and waste disposal in the endoplasmic reticulum. Bioessays 32, 905-913 (2010).

9. Bordallo, J., Plemper, R.K., Finger, A. \& Wolf, D.H. Der3p/Hrd1p is required for endoplasmic reticulum-associated degradation of misfolded lumenal and integral membrane proteins. Mol Biol Cell 9, 209-222 (1998).

10. Bays, N.W., Gardner, R.G., Seelig, L.P., Joazeiro, C.A. \& Hampton, R.Y. Hrd1p/Der3p is a membrane-anchored ubiquitin ligase required for ER-associated degradation. Nat Cell Biol 3, 24-29 (2001).

11. Kim, W., Spear, E.D. \& Ng, D.T. Yos9p detects and targets misfolded glycoproteins for ER-associated degradation. Mol Cell 19, 753-764 (2005).

12. Gauss, R., Sommer, T. \& Jarosch, E. The Hrd1p ligase complex forms a linchpin between ER-lumenal substrate selection and Cdc48p recruitment. EMBO J 25, $1827-$ 1835 (2006).

13. Gauss, R., Jarosch, E., Sommer, T. \& Hirsch, C. A complex of Yos9p and the HRD ligase integrates endoplasmic reticulum quality control into the degradation machinery. Nat Cell Biol 8, 849-854 (2006). 
14. Horn, S.C. et al. Usa1 functions as a scaffold of the HRD-ubiquitin ligase. Mol Cell 36, 782-793 (2009).

15. Denic, V., Quan, E.M. \& Weissman, J.S. A luminal surveillance complex that selects misfolded glycoproteins for ER-associated degradation. Cell 126, 349-359 (2006).

16. Hitt, R. \& Wolf, D.H. Der1p, a protein required for degradation of malfolded soluble proteins of the endoplasmic reticulum: topology and Der1-like proteins. FEMS Yeast Res 4, 721-729 (2004).

17. Taxis, C. et al. Use of modular substrates demonstrates mechanistic diversity and reveals differences in chaperone requirement of ERAD. J Biol Chem 278, 3590335913 (2003).

18. Vashist, S. \& Ng, D.T. Misfolded proteins are sorted by a sequential checkpoint mechanism of ER quality control. J Cell Biol 165, 41-52 (2004).

19. Knop, M., Finger, A., Braun, T., Hellmuth, K. \& Wolf, D.H. Der1, a novel protein specifically required for endoplasmic reticulum degradation in yeast. $E M B O \mathrm{~J} 15$, 753-763 (1996).

20. Carvalho, P., Stanley, A.M. \& Rapoport, T.A. Retrotranslocation of a misfolded luminal ER protein by the ubiquitin-ligase Hrd1p. Cell 143, 579-591 (2010).

21. Tirosh, B., Furman, M.H., Tortorella, D. \& Ploegh, H.L. Protein unfolding is not a prerequisite for endoplasmic reticulum-to-cytosol dislocation. J Biol Chem 278, 66646672 (2003).

22. de Virgilio, M., Weninger, H. \& Ivessa, N.E. Ubiquitination is required for the retrotranslocation of a short-lived luminal endoplasmic reticulum glycoprotein to the cytosol for degradation by the proteasome. J Biol Chem 273, 9734-9743 (1998).

23. $\mathrm{Yu}, \mathrm{H}$. \& Kopito, R.R. The role of multiubiquitination in dislocation and degradation of the alpha subunit of the $T$ cell antigen receptor. $J$ Biol Chem 274, 36852-36858 (1999).

24. Jarosch, E. et al. Protein dislocation from the ER requires polyubiquitination and the AAA-ATPase Cdc48. Nat Cell Biol 4, 134-139 (2002).

25. Hampton, R.Y. \& Sommer, T. Finding the will and the way of ERAD substrate retrotranslocation. Curr Opin Cell Biol 24, 460-466 (2012).

26. Bagola, K., Mehnert, M., Jarosch, E. \& Sommer, T. Protein dislocation from the ER. Biochim Biophys Acta 1808, 925-936 (2011).

27. Carvalho, P., Goder, V. \& Rapoport, T.A. Distinct ubiquitin-ligase complexes define convergent pathways for the degradation of ER proteins. Cell 126, 361-373 (2006).

28. Goder, V., Carvalho, P. \& Rapoport, T.A. The ER-associated degradation component Der1p and its homolog Dfm1p are contained in complexes with distinct cofactors of the ATPase Cdc48p. FEBS Lett 582, 1575-1580 (2008).

29. Friedlander, R., Jarosch, E., Urban, J., Volkwein, C. \& Sommer, T. A regulatory link between ER-associated protein degradation and the unfolded-protein response. Nat Cell Biol 2, 379-384 (2000).

30. Travers, K.J. et al. Functional and genomic analyses reveal an essential coordination between the unfolded protein response and ER-associated degradation. Cell 101, 249-258 (2000).

31. Stanley, A.M., Carvalho, P. \& Rapoport, T. Recognition of an ERAD-L substrate analyzed by site-specific in vivo photocrosslinking. FEBS Lett 585, 1281-1286 (2011). 
32. Greenblatt, E.J., Olzmann, J.A. \& Kopito, R.R. Derlin-1 is a rhomboid pseudoprotease required for the dislocation of mutant alpha-1 antitrypsin from the endoplasmic reticulum. Nat Struct Mol Biol 18, 1147-1152 (2011).

33. Hampton, R.Y., Gardner, R.G. \& Rine, J. Role of $26 \mathrm{~S}$ proteasome and HRD genes in the degradation of 3-hydroxy-3-methylglutaryl-CoA reductase, an integral endoplasmic reticulum membrane protein. Mol Biol Cell 7, 2029-2044 (1996).

34. Chin, J.W. et al. An expanded eukaryotic genetic code. Science 301, 964-967 (2003).

35. Chen, S., Schultz, P.G. \& Brock, A. An improved system for the generation and analysis of mutant proteins containing unnatural amino acids in Saccharomyces cerevisiae. J Mol Biol 371, 112-122 (2007).

36. Nishikawa, S.I., Fewell, S.W., Kato, Y., Brodsky, J.L. \& Endo, T. Molecular chaperones in the yeast endoplasmic reticulum maintain the solubility of proteins for retrotranslocation and degradation. J Cell Biol 153, 1061-1070 (2001).

37. Rapoport, T.A. Protein translocation across the eukaryotic endoplasmic reticulum and bacterial plasma membranes. Nature 450, 663-669 (2007).

38. Lilley, B.N. \& Ploegh, H.L. A membrane protein required for dislocation of misfolded proteins from the ER. Nature 429, 834-840 (2004).

39. Sun, F. et al. Derlin-1 promotes the efficient degradation of the cystic fibrosis transmembrane conductance regulator (CFTR) and CFTR folding mutants. $J$ Biol Chem 281, 36856-36863 (2006).

40. Ye, Y. et al. Recruitment of the p97 ATPase and ubiquitin ligases to the site of retrotranslocation at the endoplasmic reticulum membrane. Proc Natl Acad Sci U S A 102, 14132-14138 (2005).

41. Lilley, B.N. \& Ploegh, H.L. Multiprotein complexes that link dislocation, ubiquitination, and extraction of misfolded proteins from the endoplasmic reticulum membrane. Proc Natl Acad Sci U S A 102, 14296-14301 (2005).

42. Oda, Y. et al. Derlin-2 and Derlin-3 are regulated by the mammalian unfolded protein response and are required for ER-associated degradation. J Cell Biol 172, 383-393 (2006).

43. Wahlman, J. et al. Real-time fluorescence detection of ERAD substrate retrotranslocation in a mammalian in vitro system. Cell 129, 943-955 (2007).

44. Ye, Y., Shibata, Y., Yun, C., Ron, D. \& Rapoport, T.A. A membrane protein complex mediates retro-translocation from the ER lumen into the cytosol. Nature 429, 841-847 (2004).

45. Younger, J.M. et al. Sequential quality-control checkpoints triage misfolded cystic fibrosis transmembrane conductance regulator. Cell 126, 571-582 (2006).

46. Stolz, A., Hilt, W., Buchberger, A. \& Wolf, D.H. Cdc48: a power machine in protein degradation. Trends Biochem Sci 36, 515-523 (2011).

47. Sato, B.K., Schulz, D., Do, P.H. \& Hampton, R.Y. Misfolded membrane proteins are specifically recognized by the transmembrane domain of the Hrd1p ubiquitin ligase. Mol Cell 34, 212-222 (2009).

\section{Methods}

Antibodies

Monoclonal anti-HA (H9658, Sigma-Aldrich, St. Louis, USA; C29F4, NEB, UK) and anti-Myc (M5546, Sigma-Aldrich, St. Louis, USA) antibodies used for immunoprecipitation (dilution 
1:2,000) and detection (dilution 1:2,500 - 1:5,000) were commercially available. Antibodies for immunodetection of Hrd1 (1:10,000), Hrd3 (1:3,000), Sec61 (1:10,000), Der1 (1:500) and Usa1 $(1: 3,000)$ were described previously ${ }^{12,}{ }^{14}$. Antibodies for immunodetection of Kar2 $(1: 10,000)$ and Pep4 $(1: 3,000)$ were a generous gift from T.A. Rapoport and D.H. Wolf, respectively. The monoclonal antibody directed against CPY (A-6428; used 1:2,500 for immunoprecipitation and 1:5,000 for immunodetection) was purchased from Molecular Probes. Fluorescently labelled secondary antibodies (IRDye 800, anti-mouse IgG (goat) and IRDye 800, anti-rabbit (goat); each used 1:2,000 for immunotecetion by LiCor) were purchased from Rockland. The polyclonal anti-Yos9 antibody was generated by immunising rabbits with a fragment of Yos9 purified from E. coli and used at a dilution of 1:2,000 for immunodetection. The polyubiquitin-specific antibody (UG9510, Enzo Life Science) was diluted 1:2,000 in immunoblotting. Horseradish-peroxidase coupled secondary antibodies (A9044 and A0545; both diluted 1:10,000) used for immunodetection of proteins by Enhanced Chemiluminiscence were purchased from Sigma-Aldrich, St. Louis.

\section{Yeast strains and plasmids}

For yeast sporulation, tetrad dissection and the preparation of yeast media standard protocols were used ${ }^{48}$. The genotypes of yeast strains are listed in the Supplementary Table 2. These strains are derived from the diploid wild-type strain DF5. Described methods were followed for the deletion and chromosomal epitope tagging of genes in yeast ${ }^{49,50}$. DNAplasmids used in this study are summarised in the Supplementary Table 3. Overall, a DNA fragment was PCR-amplified with oligonucleotides that are flanked by suitable restriction sites and inserted into appropriate vectors. The modification of plasmid-encoded genes with epitopes (pMM074, pMM075 and pMM076) was done by expressing these constructs in the respective deletion strains and homologues recombination of an appropriate DNA-cassette as described ${ }^{50}$. Der1 constructs containing point mutations were generated by QuikChange site-directed mutagenesis using Pfu High Fidelity DNA-polymerase according to the manufacturer's instructions (Agilent Technologies, USA). For the generation of constructs that express p-Benzoylphenylalanine- (pBpa-) labelled Der1 variants pMM074, pMM075 and pMM063 were used as parental plasmids. The stop codon TAG was introduced at individual positions by site-directed mutagenesis as described above. pMM001, pMM040 and pMM041 were used for the insertion of point mutations into the chromosomal locus of Der1 leading to YMM156, YMM111 and YMM112. 6xMyc-Hmg2 was expressed from plasmid pRH244 as described elsewhere ${ }^{33}$. pJU293 and pJU294 are $2 \mu \mathrm{m}$-based multicopy plasmids that contain combinations of the genes HRD1, HRD3 and DER1 under the control of their respective promoters (around $400 \mathrm{bp}$ of the 5 ' non-coding sequence). 
Native immunoprecipitation

$50 \mathrm{OD}_{600}$ yeast cells in early logarithmic phase were harvested and lysed by vigorous shaking with glass beads in ice-cold lysis buffer $(50 \mathrm{mM}$ Tris- $\mathrm{HCl}(\mathrm{pH} 7.5), 400 \mathrm{mM} \mathrm{KOAc}$, 0,5 mM EDTA, $10 \%$ (v/v) Glycerol, $1 \mathrm{mM}$ phenymethyl sulphonyl fluoride (PMSF)). The cell debris was removed by low-speed centrifugation $(2,000 \times \mathrm{g}, 3 \mathrm{~min})$. The supernatant was supplemented with $1 \%$ (w/v) Digitonin (Calbiochem, USA) and incubated for 1 hour under rotation at $4{ }^{\circ} \mathrm{C}$. The lysate was cleared by high-speed centrifugation at $20,800 \times \mathrm{g}$ for $20 \mathrm{~min}$ and diluted with lysis buffer to a final concentration of $0.5 \%(w / v)$ Digitonin. For immunoprecipitation specific antibodies and Protein A-conjugated Sepharose were added. Bound proteins were eluted with dithiothreitol (DTT) containing sample buffer and analysed by Sodiumdodecyl-polyacrylamide gel electrophoresis (SDS-PAGE) and immunoblotting. For the immunoprecipitation of $\mathrm{CPY}^{*}$ the protocol was modified. $150 \mathrm{OD}_{600}$ yeast cells were harvested and lysed in IP32-buffer (50 mM HEPES ( $\mathrm{pH} 7.5$ ), $50 \mathrm{mM} \mathrm{NaCl}, 125 \mathrm{mM} \mathrm{KOAc,} 2$ $\mathrm{mM} \mathrm{MgOAc} 2,1 \mathrm{mM}$ EDTA, $10 \mu \mathrm{M} \mathrm{CaCl}_{2}, 3 \%$ (v/v) Glycerol). Next, microsomes were collected by high-speed centrifugation $(20,800 \times \mathrm{g}, 20 \mathrm{~min})$ and solubilised with $0.5 \%(\mathrm{v} / \mathrm{v})$ Nonidet P40. The following steps were performed as described above.

\section{Determination of protein degradation}

The turnover of proteins were either analysed by pulse chase experiments or cycloheximide decay assays as described elsewhere ${ }^{51-53}$. Briefly, for pulse chase analysis yeast cells were pulsed with radiolabelled ${ }^{35} \mathrm{~S}$ methionine followed by a chase with an excess of unlabelled amino acids. During the chase samples were taken at indicated time points and mixed with $\mathrm{NaN}_{3}$. The cells were lysed under denaturing conditions and the protein of interest was immunoprecipitated with specific antibodies. For the analysis of $\mathrm{CPY}^{*}$ or $\operatorname{PrA}^{*}$ the bound proteins were treated with Endoglycosidase F. The samples were then separated by SDSPAGE followed by autoradiography using a Phospholmager (Typhoon FLA9500, GE Healthcare).

For a cycloheximide decay assay yeast cells were incubated with $0.3 \mathrm{mg} / \mathrm{ml}$ cycloheximid in order to stop protein synthesis. Samples were collected at indicated time points and the cells were lysed with glass beads in buffer containing $50 \mathrm{mM}$ Tris- $\mathrm{HCl}[\mathrm{pH}$ 7.5], $10 \mathrm{mM}$ EDTA, 1 mM PMSF. Microsomes were recovered by high-speed centrifugation (20,800 x g, $20 \mathrm{~min}$ ) and suspended in sample buffer. The analysis of the samples was carried out by SDS-PAGE and immunoblotting.

\section{Detection of ubiquitylated $\mathrm{CPY}^{*}$}

$150 \mathrm{OD}_{600}$ yeast cells were harvested and washed with $20 \mathrm{mM} \mathrm{N}$-ethylmaleimide (NEM), 10 $\mathrm{mM} \mathrm{NaN}_{3}$ and $1 \mathrm{mM}$ PMSF. After lysis of the cells with glass beads in Ub-buffer $(0.7 \mathrm{M}$ 
sorbitol, $50 \mathrm{mM}$ Tris-HCl (pH 7.5), $150 \mathrm{mM} \mathrm{NaCl}, 5 \mathrm{mM}$ EDTA, 1 \% (v/v) Triton-X 100, 0,1\% (w/v) SDS) supplemented with $20 \mathrm{mM} \mathrm{NEM}$ and $1 \mathrm{mM} \mathrm{PMSF}$ the cell extract was incubated for 1 hour for efficient solubilisation of membranes. Insoluble material was then cleared from the lysate $(20,800 \times \mathrm{g}, 20 \mathrm{~min})$. Subsequently, CPY*-Myc was precipitated with anti-Myc antibodies. Bound proteins were eluted by DTT containing sample buffer and analysed by SDS-PAGE and immunoblotting. Ubiquitylated $\mathrm{CPY}^{*}-\mathrm{Myc}$ was detected by specific antiubiquitin antibodies.

\section{Site-specific in vivo photocrosslinking}

Yeast cells were transformed with pGK1-pBpa (a generous gift from P.G. Schultz) and Der1Myc constructs derived from pMM074 or pMM075 containing an amber stop codon at specific positions ${ }^{34,35}$. Transformed yeast cells were grown in synthetic minimal medium supplemented with $400 \mu \mathrm{M} \mathrm{pBpa}\left(\mathrm{Bachem}, \mathrm{CH}\right.$ ) over night at $30^{\circ} \mathrm{C}$. The expression of Der1Myc was induced by addition of $0.1 \mathrm{mM} \mathrm{CuSO}_{4}$ for 2 hours. $150 \mathrm{OD}_{600}$ yeast cells were harvested and washed twice with ice-cold $\mathrm{H}_{2} \mathrm{O}$. Next, the cells were suspended in $2 \mathrm{ml} \mathrm{H}_{2} \mathrm{O}$, transferred to a 6-well plate and irradiated with UV light (B-100AP, UVP, CA) at $\lambda=365 \mathrm{~nm}$ for $45 \mathrm{~min}$ on ice. The cells were lysed with glass beads in $50 \mathrm{mM}$ Tris- $\mathrm{HCl}(\mathrm{pH} 7.5), 150 \mathrm{mM}$ $\mathrm{NaCl}, 5 \mathrm{mM}$ EDTA, 1 \% (v/v) Triton X-100, $0.1 \%$ (w/v) SDS, 1 mM PMSF. The lysate was cleared from cell debris and the microsomes were solubilised as described above. Insoluble material was removed by high-speed centrifugation (20,800 x g, $20 \mathrm{~min})$ and Der1-Myc was immunoprecipitated with anti-Myc antibodies. Bound proteins were eluted with DTTcontaining sample-buffer and analysed by SDS-PAGE and immunoblotting. For quantification of the crosslinking efficiency immunoblots were incubated with fluorescently labelled secondary antibodies and analysed by the Odyssey near-infrared Imaging System (Li-Cor).

\section{Protease protection assay}

To monitor the dislocation of a CPY fraction from the ER over a time period of 1 hour the protein synthesis in yeast cells was inhibited by addition of cycloheximide as described before. Samples were collected at the indicated time points and washed with $10 \mathrm{mM} \mathrm{NaN}_{3}$. To keep the membranes intact the cells were lysed in sorbitol-buffer $(0.7 \mathrm{M}$ sorbitol, $50 \mathrm{mM}$ Tris- $\mathrm{HCl}(\mathrm{pH} 7.5))$. Afterwards, the lysate was cleared from cell debris and treated with 0.1 $\mathrm{mg} / \mathrm{ml}$ proteinase $\mathrm{K}$, or proteinase $\mathrm{K}$ in presence of $0.5 \%(\mathrm{v} / \mathrm{v})$ Triton-X 100 for $20 \mathrm{~min}$ on ice. The reaction was stopped by the addition of $1 \mathrm{mM} \mathrm{PMSF}$ and $15 \%(\mathrm{w} / \mathrm{v})$ trichloro-acetic acid. The proteins were then recovered by high-speed centrifugation $(20,800 \times \mathrm{g}, 20 \mathrm{~min})$ and dissolved in sample buffer $(\mathrm{pH} 8)$ before analysis by SDS-PAGE and immunoblotting. 
For the measurement of UPR induction yeast cells were transformed with a UPR-induced reporter construct (pUPRE-lacZ). The ß-galactosidase activity was determined as previously described ${ }^{29}$.

Reproducability of experiments

All data are representative of at least three independent experiments with the exception of Figures 1c (two independent experiments), Supplementary Figures 1c, 1e, 2b right panel, 2c, 3a, 3c, 4c and 5a (two independent experiments each) and Supplementary Fig. 3b (one experiment). Uncropped images of key experiments can be seen in Supplementary Fig. 6.

\section{References}

48. Ausubel, F.M.e. (ed.) Current Protocols in Molecular Biology. (2007).

49. Knop, M. et al. Epitope tagging of yeast genes using a PCR-based strategy: more tags and improved practical routines. Yeast 15, 963-972 (1999).

50. Longtine, M.S. et al. Additional modules for versatile and economical PCR-based gene deletion and modification in Saccharomyces cerevisiae. Yeast 14, 953-961 (1998).

51. Biederer, T., Volkwein, C. \& Sommer, T. Degradation of subunits of the Sec61p complex, an integral component of the ER membrane, by the ubiquitin-proteasome pathway. EMBO J 15, 2069-2076 (1996).

52. Biederer, T., Volkwein, C. \& Sommer, T. Role of Cue1p in ubiquitination and degradation at the ER surface. Science 278, 1806-1809 (1997).

53. Walter, J., Urban, J., Volkwein, C. \& Sommer, T. Sec61p-independent degradation of the tail-anchored ER membrane protein Ubc6p. EMBO J 20, 3124-3131 (2001). 


\section{Figure-1 (Jarosch)}
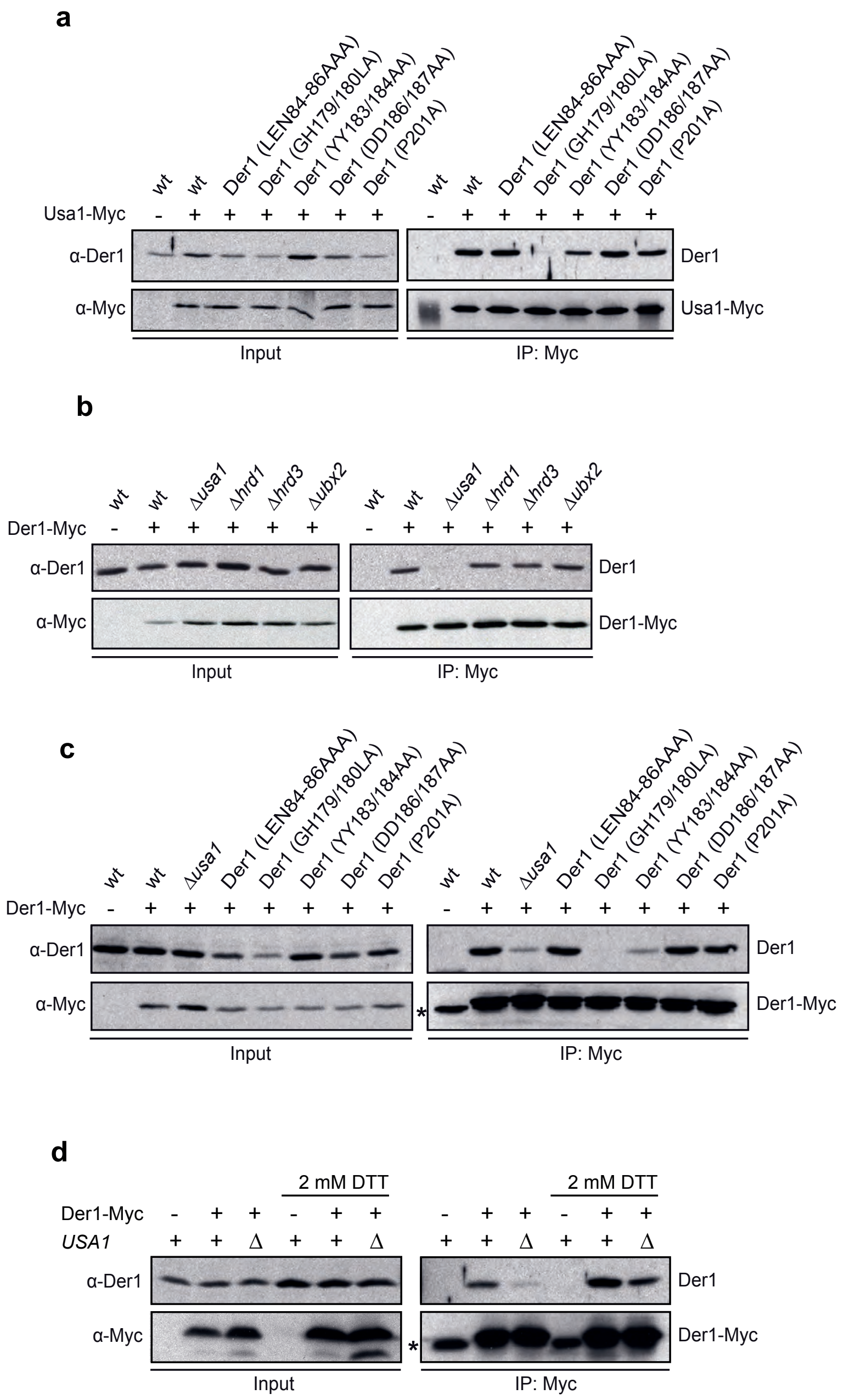


\section{Figure-2 (Jarosch)}

a

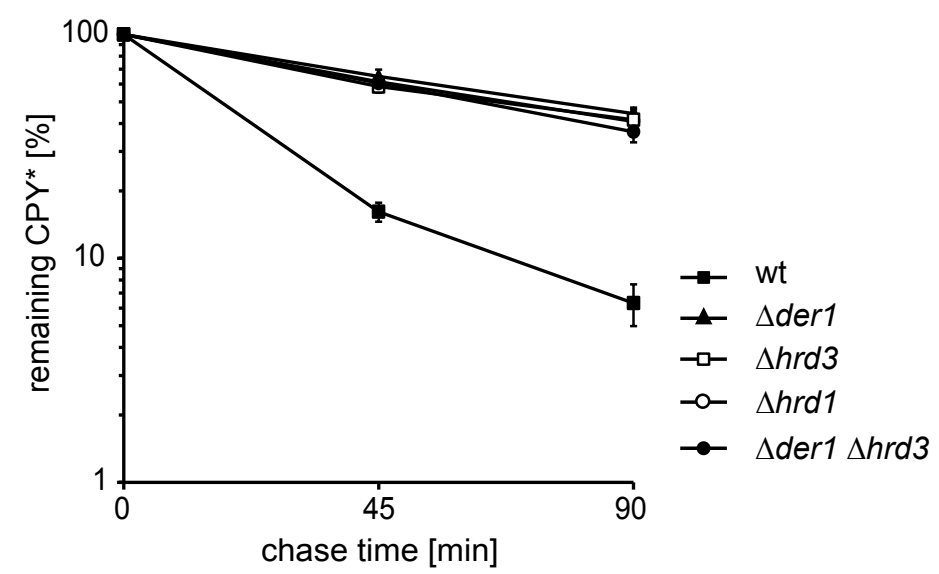

C

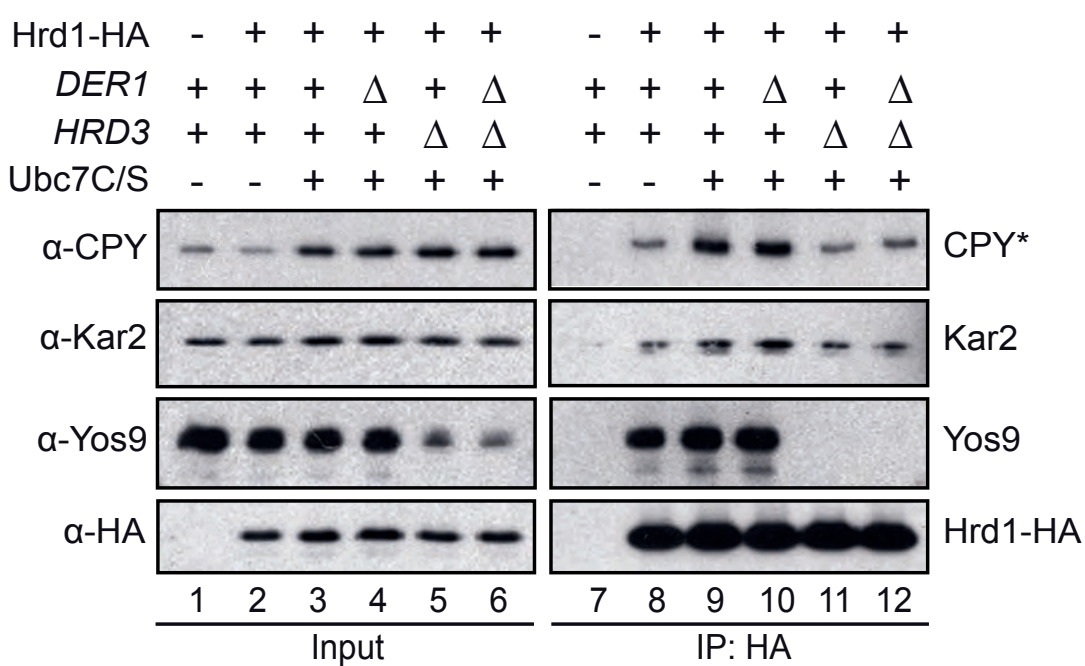

b

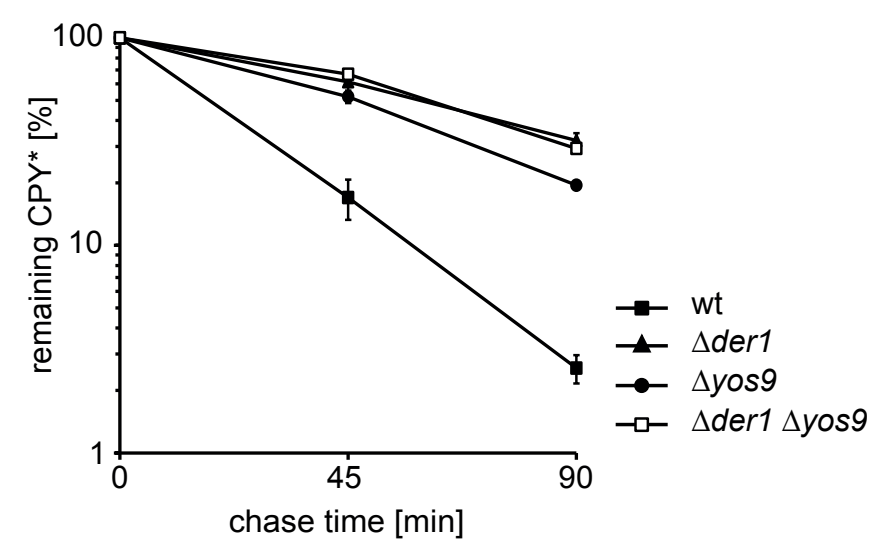

d

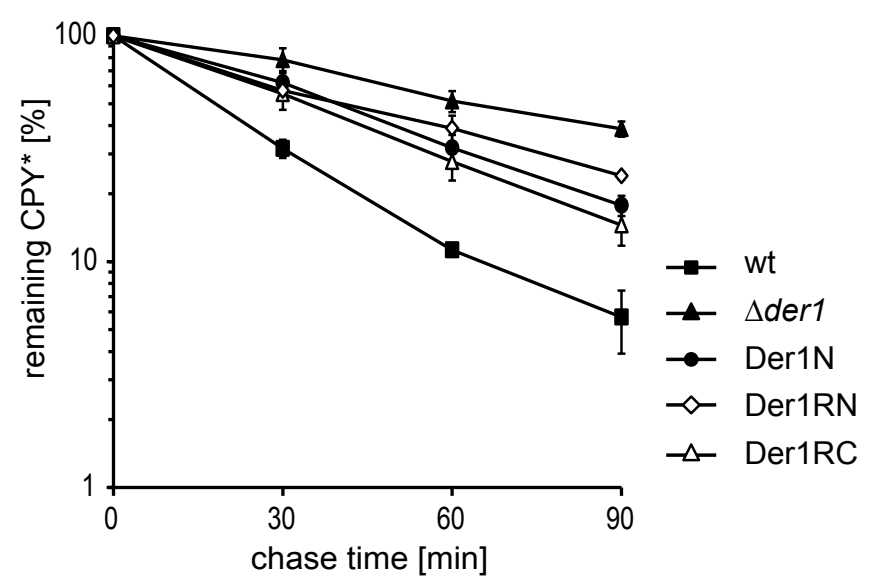

e

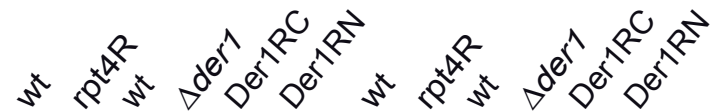

CPY*-Мус - $+++++\ldots++++$

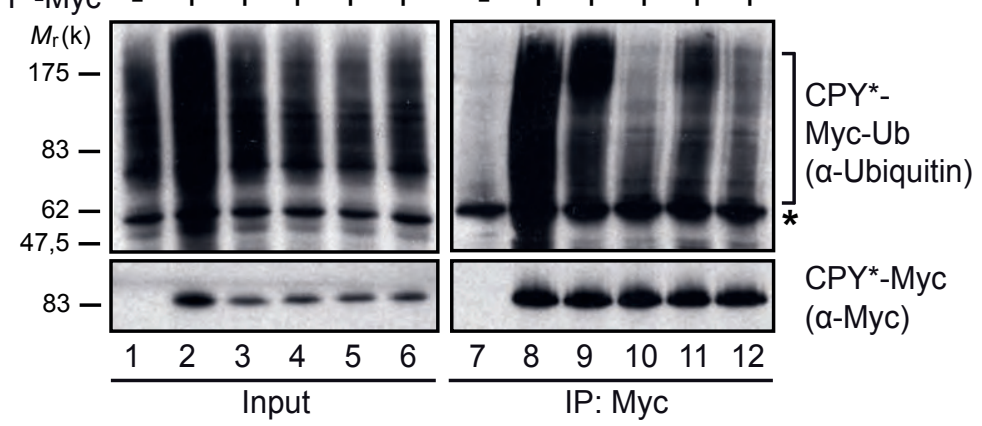

\section{f}

Triton-X $100 \quad-\quad-\quad-\quad-\quad+\quad+\quad-\quad-\quad-\quad+\quad+$

Proteinase $\mathrm{K}-\mathrm{C}_{-}+++-\quad++++$

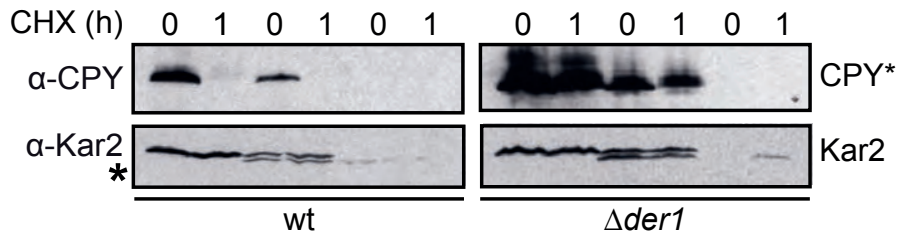

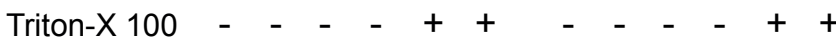

Proteinase $\mathrm{K}-\mathrm{C}_{-}++-\mathrm{C}_{+}++$

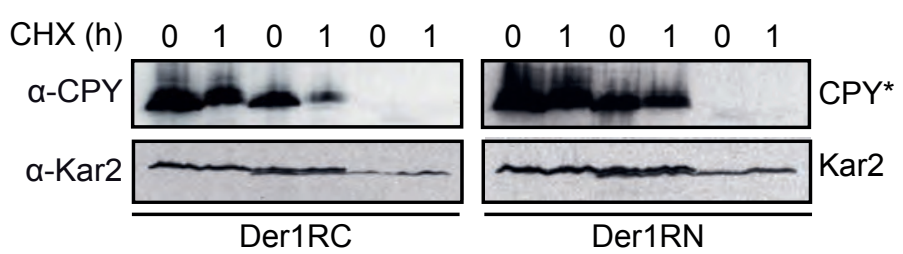


Figure-3 (Jarosch)
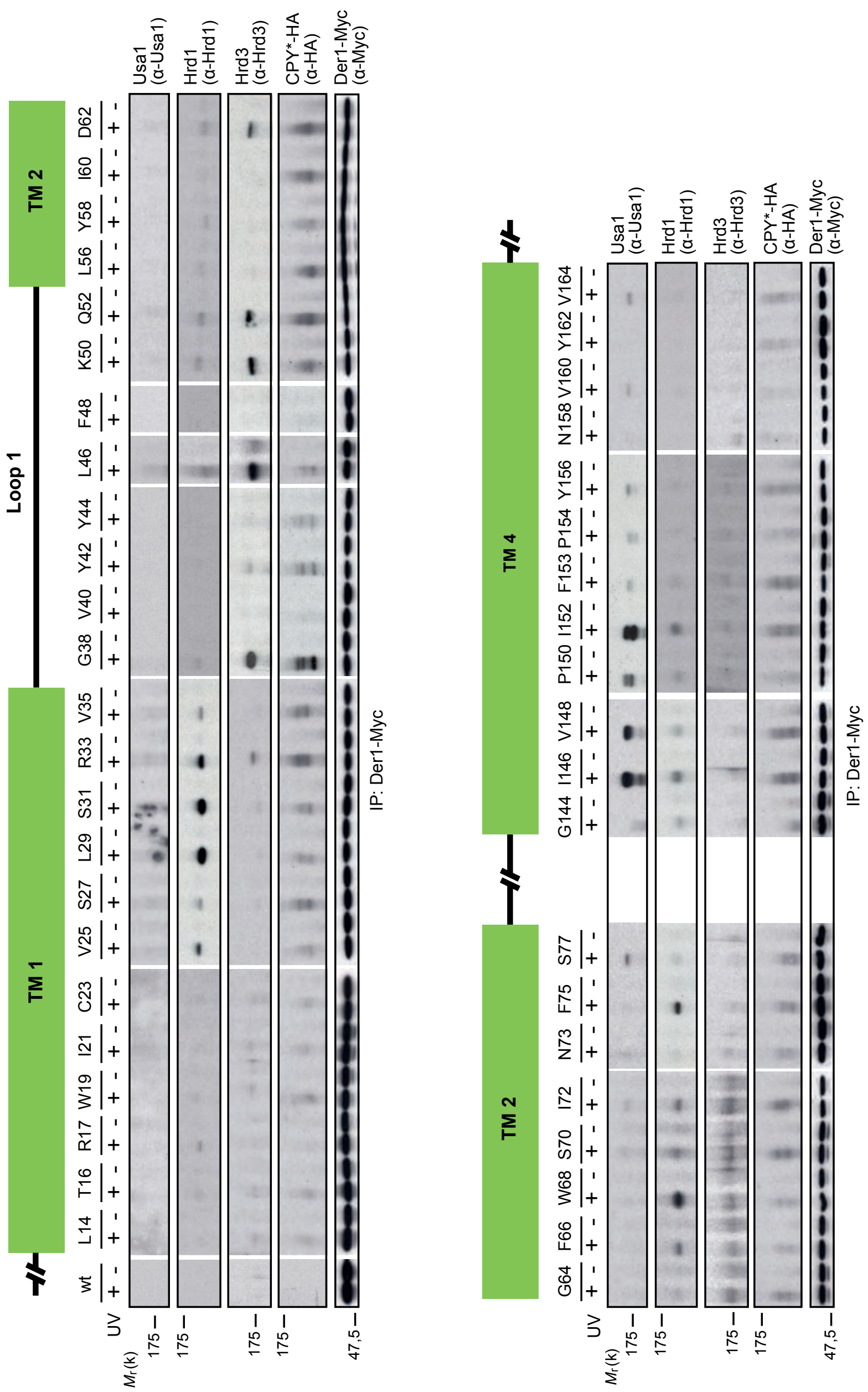
Figure-4 (Jarosch)

a

$$
\text { USA1 } \frac{\text { S31 }}{+} \frac{\Delta}{+-\frac{1}{+-}} \frac{\Delta}{+-}
$$

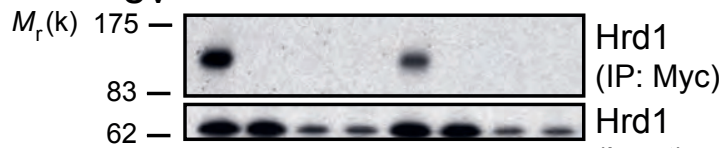

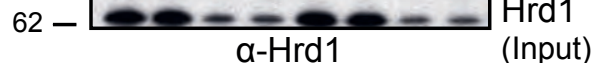

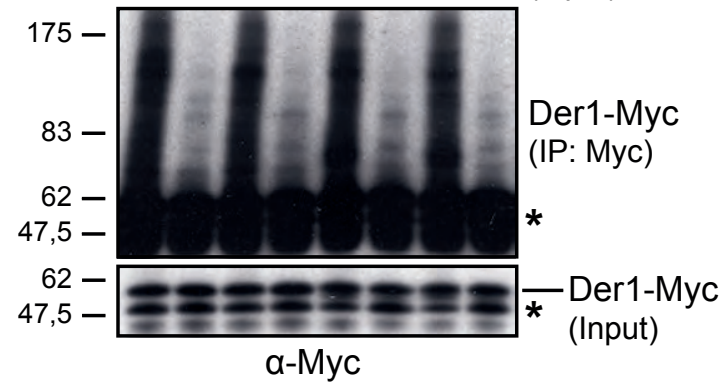

C

$\frac{\mathrm{R} 33}{\mathrm{Hrd3} 3} \frac{\mathrm{G} 38}{\mathrm{Hrd3}} \frac{\mathrm{L} 46}{\mathrm{Hrd3}}$

UV $\frac{w t}{+-} \frac{(1-769)}{+-} \frac{\mathrm{wt}}{+-} \frac{(1-769)}{+-} \frac{\mathrm{wt}}{+-} \frac{(1-769)}{+-}$

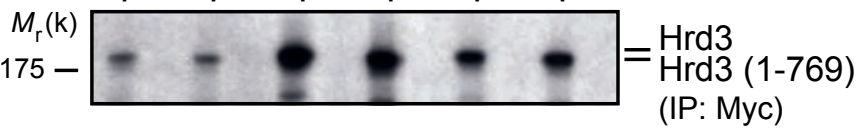

$$
\begin{gathered}
\mathrm{\alpha}-\mathrm{Hrd} 3 \\
83-\ldots \mathrm{Hrd3} \\
\text { (Input) }
\end{gathered}
$$

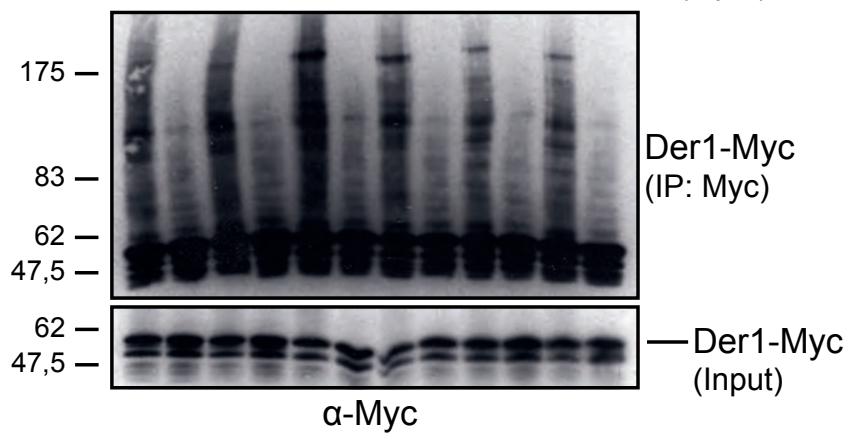

b

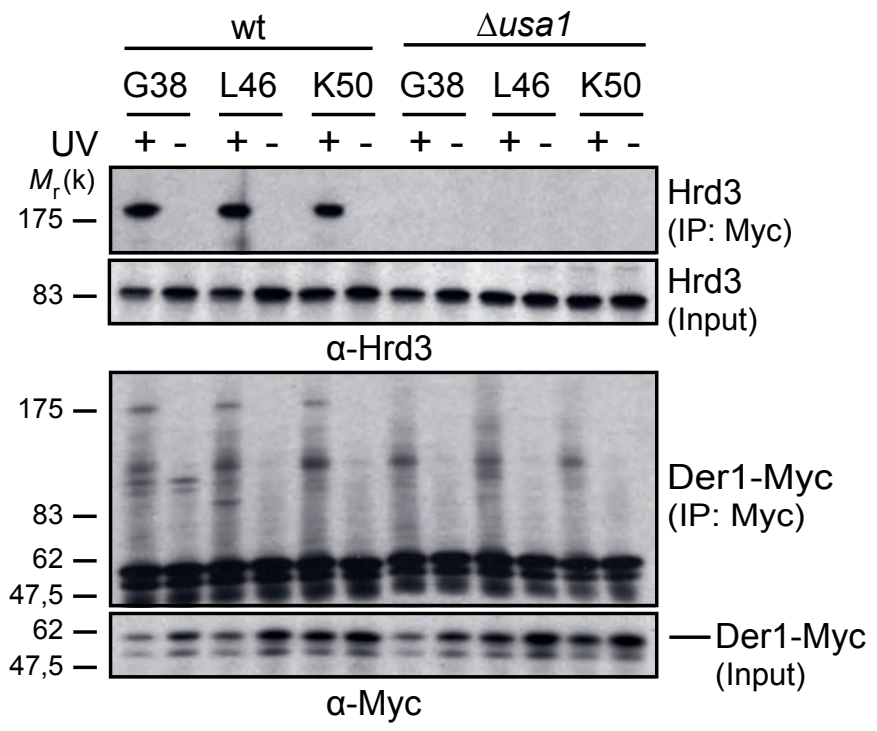

d

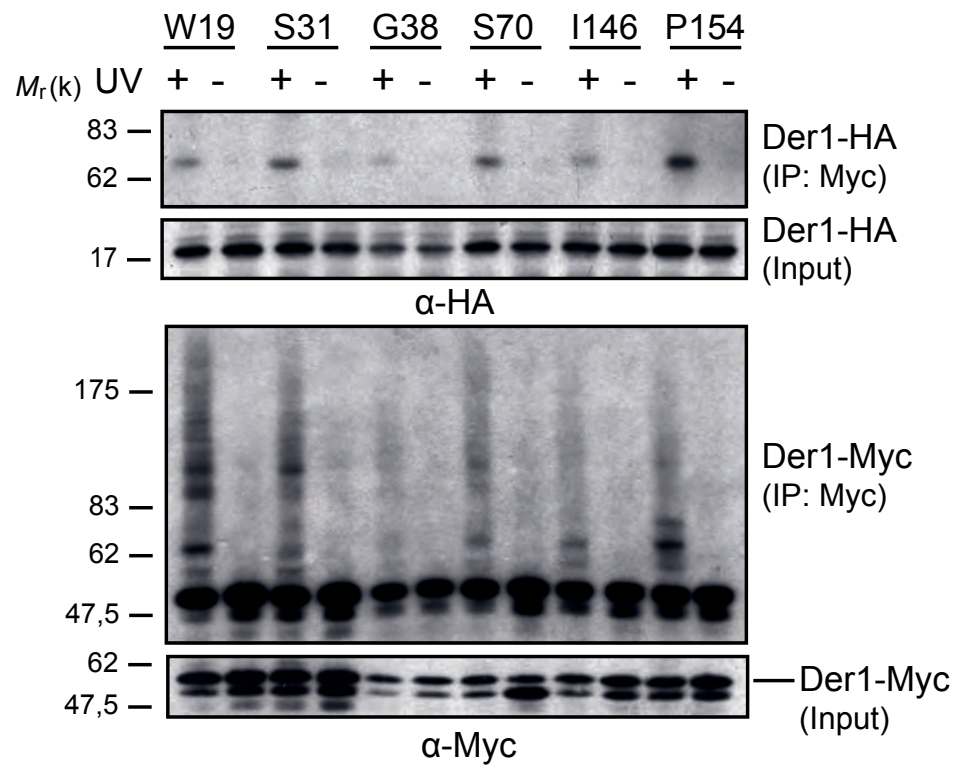


Figure-5 (Jarosch)

a

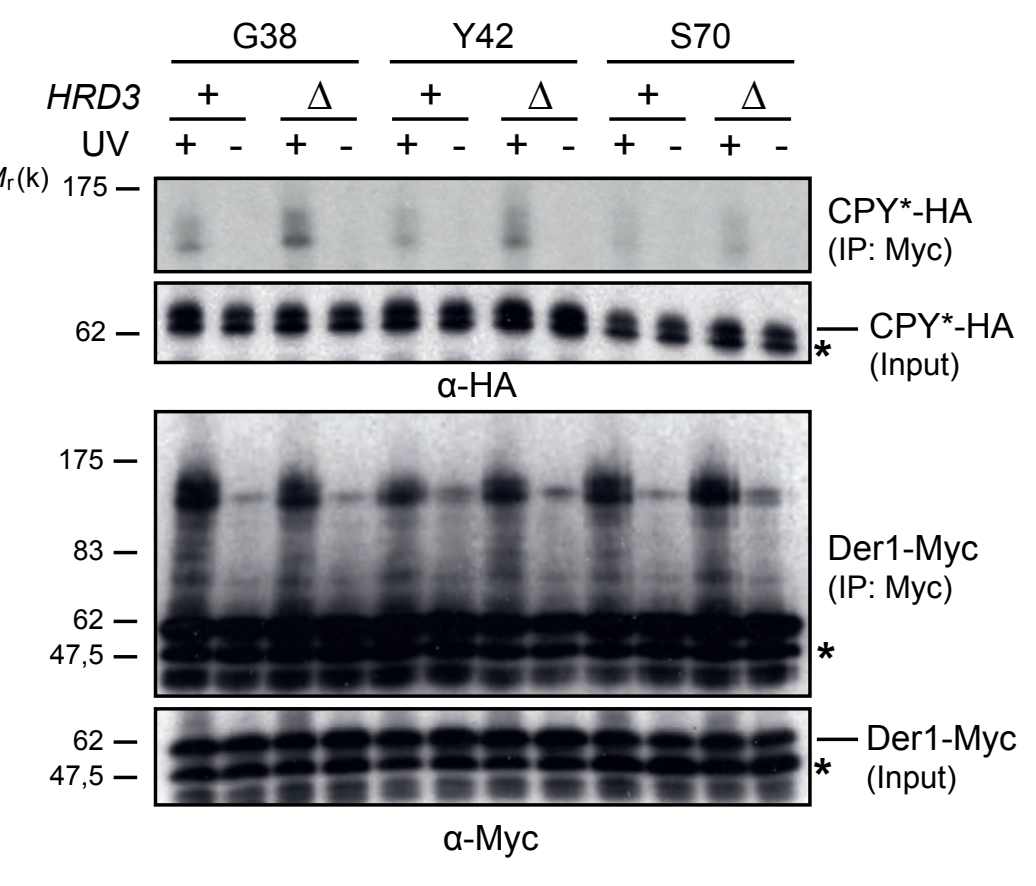

b

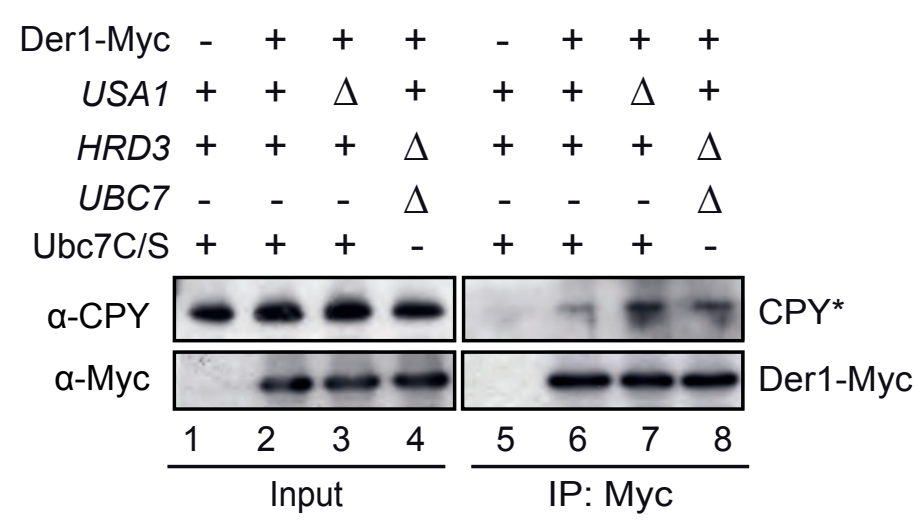

d

C

$\frac{\text { wt }}{\frac{\mathrm{G} 38}{+-}} \frac{\mathrm{D} 62}{+-} \frac{\mathrm{S} 70}{+} \frac{\mathrm{G} 38}{+-} \frac{\mathrm{D} 62}{+} \frac{\mathrm{S} 70}{+-}$

$M_{\mathrm{r}}(\mathrm{k})$

175
83

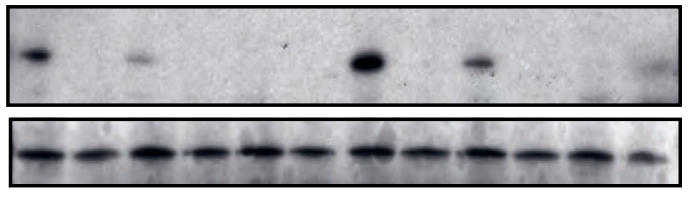

$\mathrm{Hrd} 3$ (IP: Myc)

Hrd3

(Input)

a-Hrd3

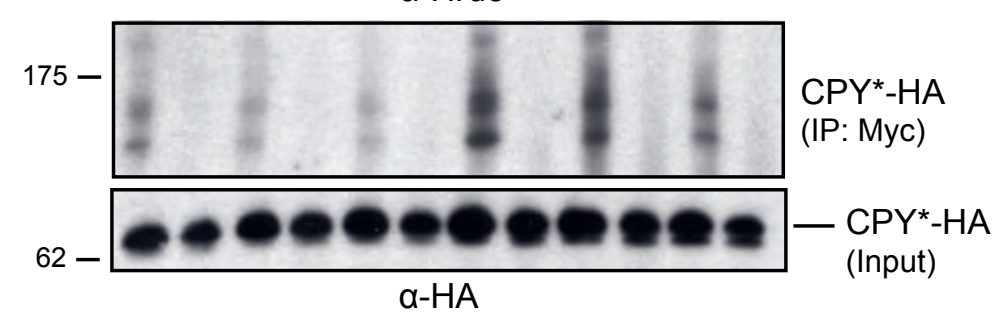

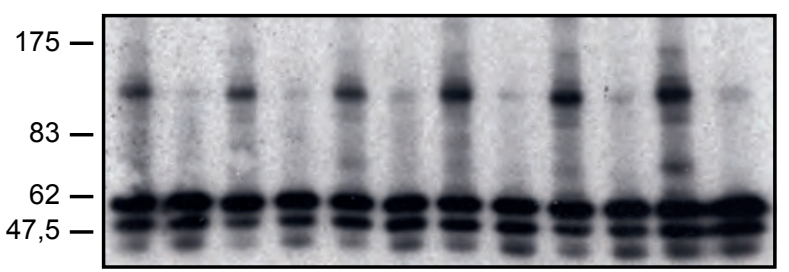

62

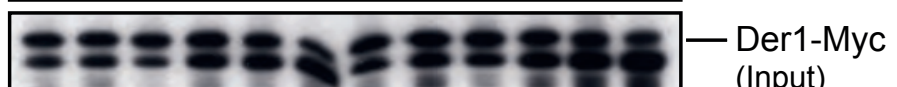

a-Myc
Der1-Myc (IP: Myc) (Input)

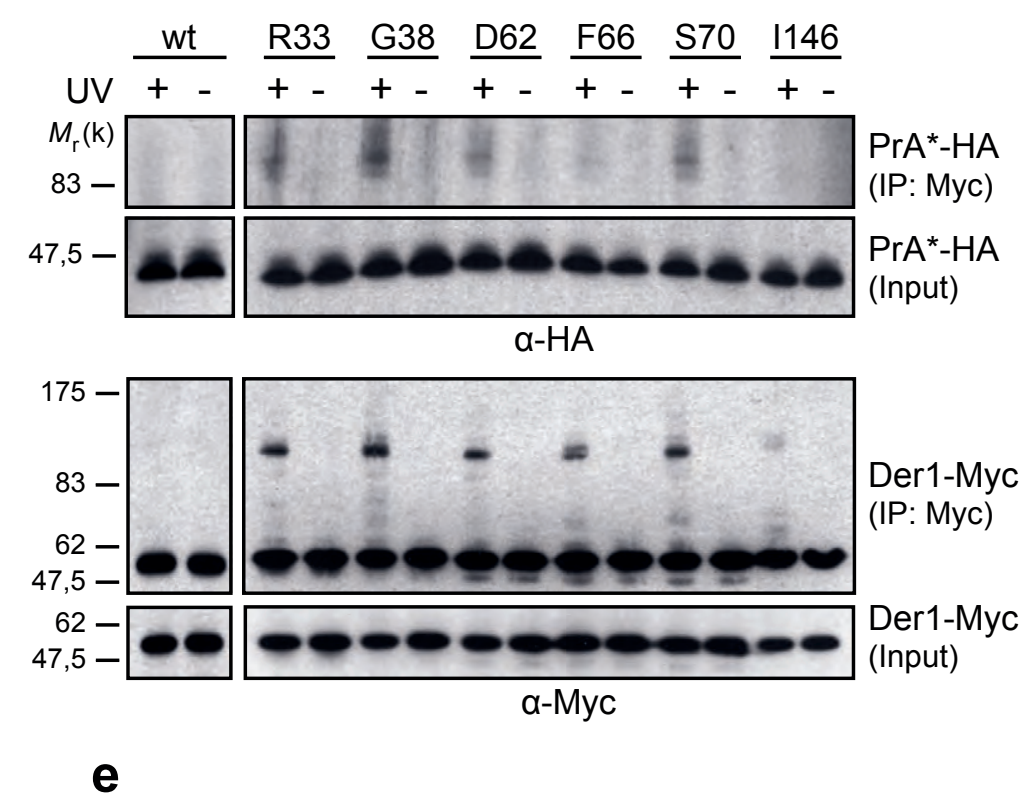

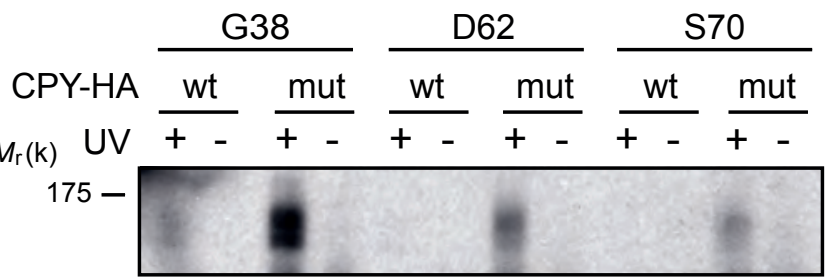

CPY-HA variants (IP: Myc)

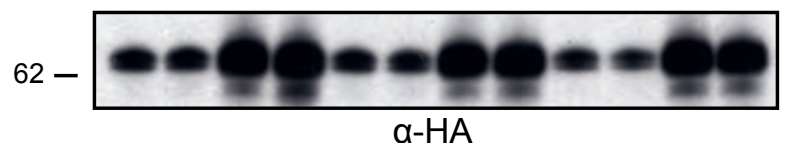
CPY-HA variants (Input)

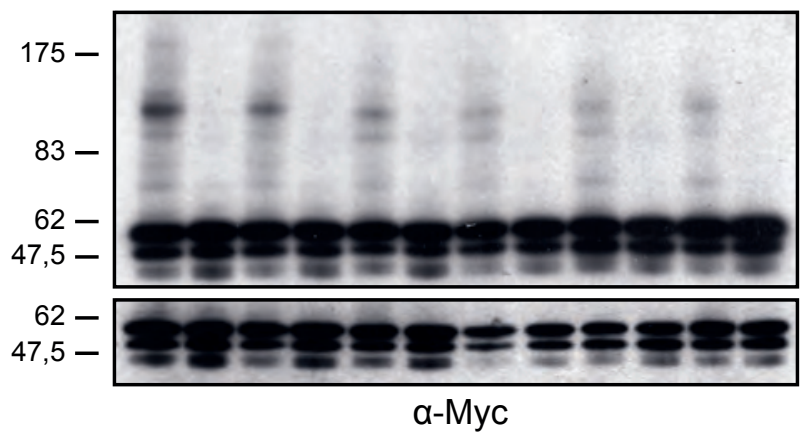

Der1-Myc (IP: Myc)

-Der1-Myc (Input) 


\section{Figure-6 (Jarosch)}

a

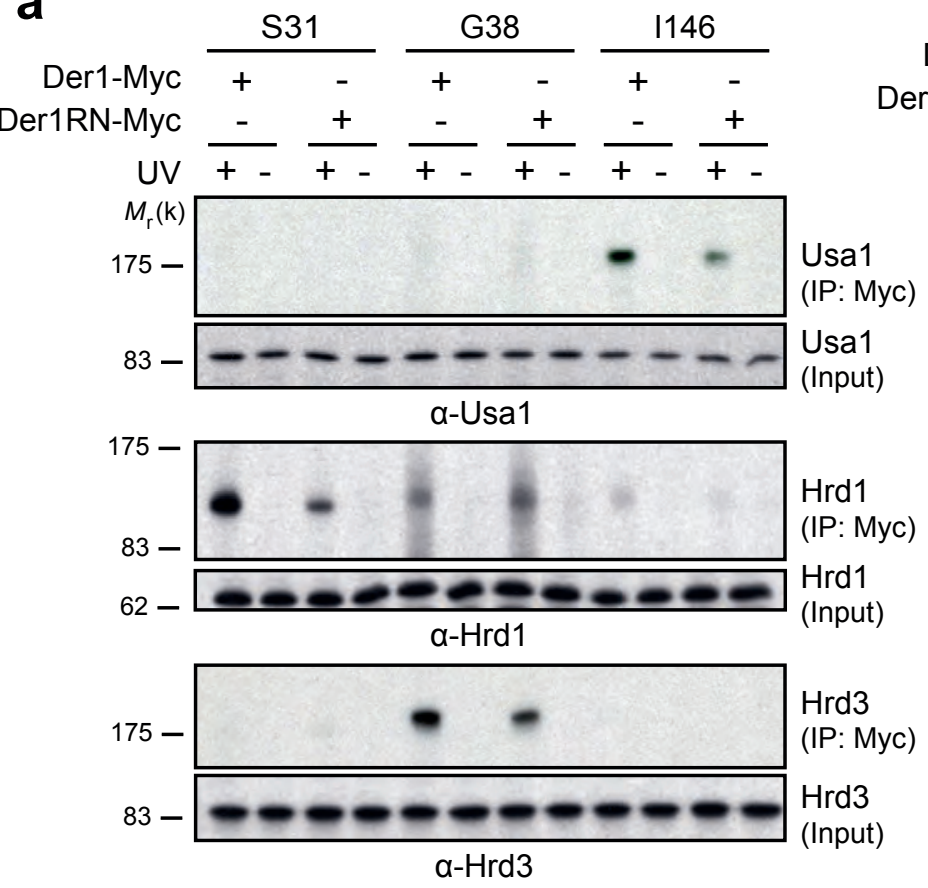

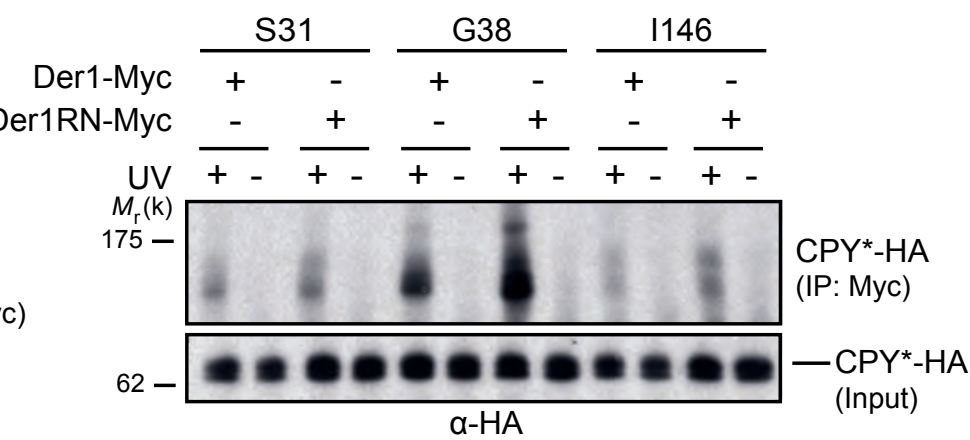

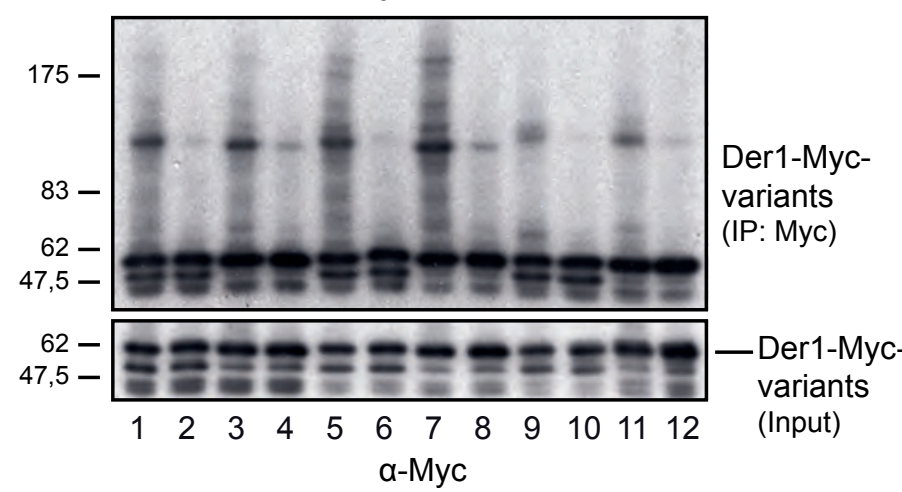

b

Der1-Myc $\frac{\text { W68 }}{+} \frac{\text { F75 }}{+}$

$\underset{M_{\mathrm{r}}(\mathrm{k})}{\operatorname{Der} 1 \mathrm{UV}} \frac{-}{+-} \frac{+}{+-} \frac{-}{+-} \frac{+}{+-}$
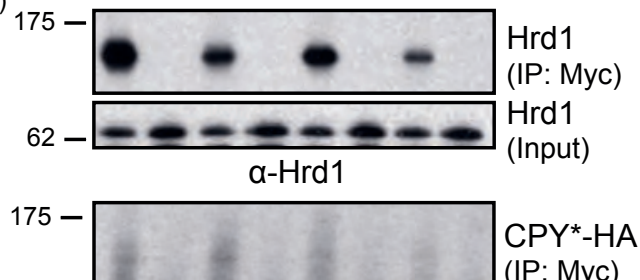

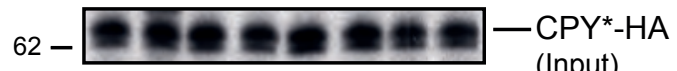

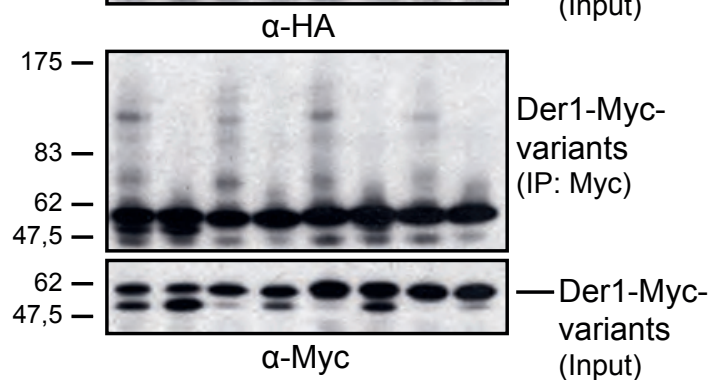

C

Der1-Myc $\frac{\mathrm{S} 31}{+} \frac{\mathrm{G} 38}{+-\frac{1146}{+}-}$

Der1RN-Myc $\frac{-}{M_{1}(\mathrm{k})}$ UV $+\frac{-}{+-} \frac{+}{+-}+\frac{+}{+-}$ $M_{r}(\mathrm{k})$

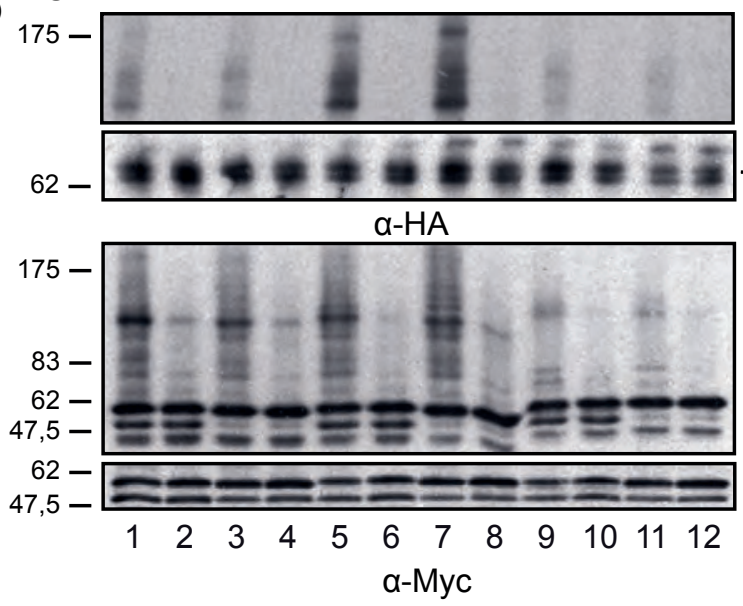

CPY*-HA (IP: Myc)

- ${ }_{(\text {Input })}^{-\mathrm{HA}}$

Der1-Myc-

variants

(IP: Myc)

-Der1-Mycvariants (Input) 
Figure-7 (Jarosch)

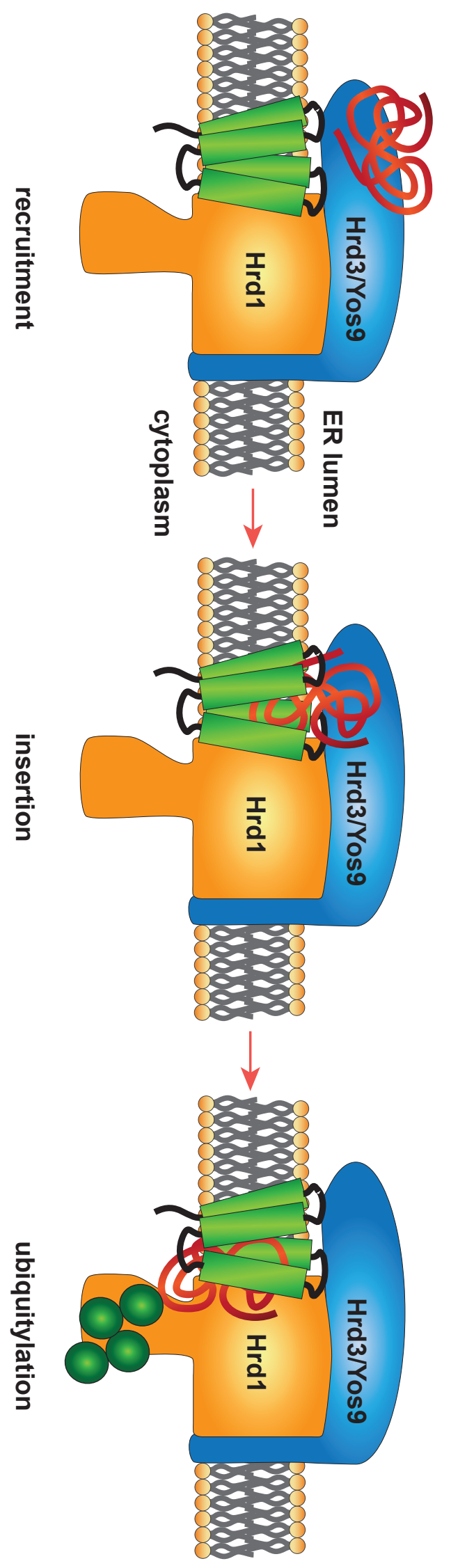




\section{Supplementary material}

\section{Supplementary Figure 1}

The carboxyterminus integrates Der1 into the HRD-ligase

a, Sequence alignment of yeast Der1 with homologues in other organisms, generated by ClustalW and Jalview. Derlin-1 from Homo sapiens (hs, UniProtKB accession number Q9BUN8), Caenorhabditis elegans (ce, Q93561), Derlin-2 from Homo sapiens (hs, Q9GZP9), Caenorhabditis elegans (ce, Q21997), Derlin-3 from Homo sapiens (hs, Q96Q80), Mus Musculus (mm, Q9D8K3), Der1 from Saccharomyces cerevisiae (sc, P38307). The position of the Der1 transmembrane segments as predicted by hydrophobicity calculations and biochemical analysis by Hitt et al. ${ }^{16}$ is given by green bars. Of note, in an alternative model for Derlin-1 topology proposed by Greenblatt et al. ${ }^{32}$, the position of transmembrane segments one and two is almost identical. Black diamonds label amino acids in Der1, which were subjected to site-directed mutagenesis (Supplementary Table S1). b, Cycloheximide decay assay to monitor the degradation of Der1 in strains of the indicated genotypes. c, As in b but $\Delta d e r 1$ cells were transformed with low-copy number plasmids encoding mutants of Der1. d, Cycloheximide decay decay assay to determine turnover of Der1-Myc and Der1 in $\Delta u s a 1$ cells. The integral ER-membrane protein Sec61 served as loading control. e, Plasmidencoded HA-tagged Der1 was expressed with endogenous Der1 in cells containing or lacking Usa1. Membranes of the total extract were solubilised with Digitonin and Der1-HA was precipitated with anti-HA antibodies followed by SDS-PAGE and immunoblotting.

\section{Supplementary Figure 2}

Characterisation of the dislocation deficient Der1 transmembrane mutants.

a, Wt and $\triangle$ der 1 cells were transformed with high-copy plasmids encoding HRD1 and HRD3 (pJU293) or HRD1, HRD3 and DER1 (pJU294). The turnover of CPY* was determined by radioactive pulse chase analysis and the results quantified using a Phospholmager.b, Pulse chase experiment to analyse the effect of the Der1 transmembrane mutants on the degradation of $\mathrm{PrA}^{*}$ (left panel) and 6xMyc-Hmg2 (right panel). Error bars represent the standard deviation of three independent experiments. c, Cycloheximide decay assay to monitor the stability of the Der1 transmembrane mutants. The asterisk denotes a loss of cell material during the sample preparation.d, Digitonin-solubilised lysates from cells expressing Usa1-Myc and the indicated Der1 variants were subjected to immunoprecipitation with antiMyc antibodies (left panel). Vice versa cells expressing the indicated variants of Der1-Myc were lysed and tested for interaction to different components of the HRD-ligase by immunoprecipitation with anti-Myc antibodies (right panel). The bound proteins were analysed by SDS-PAGE and immunoblotting using specific antibodies.e, Cells expressing Der1-Myc were transformed with low-copy number plasmids encoding either Der1 or Der1 
transmembrane mutants were lysed in Digitonin buffer and subjected to precipitation with anti-Myc antibodies.

\section{Supplementary Figure 3}

The pBpa-labelled Der1 variants are properly integrated into the HRD-ligase.

a, Der1-Myc labelled at positions in the first (W19) and second (S70) transmembrane domain as well as in the first luminal loop (G38, Y42, L46, K50) (derived from pMM075) were expressed in $\triangle d e r 1 \mathrm{Ubc7} \mathrm{C} / \mathrm{S} \mathrm{CPY}^{*}$-HA cells to investigate crosslinking to Yos9.b, Efficiency of the CPY*-HA crosslinking to different positions in Der1-Myc. Photoreactive probes were introduced at the indicated positions of Der1-Myc (derived from pMM075) and the crosslinking experiment was performed as described (see Methods). Crosslinked CPY*-HA and precipitated pBpa-labelled Der1-Myc were detected by fluorescently labelled secondary antibodies using the Odyssey near-infrared scanner (Li-Cor) and quantified by Odyssey Imaging System Version 3.0. The amount of the CPY*-HA crosslinking at position G38 was set to $100 \%$. The efficiency of the CPY*-HA crosslinking at other positions was calculated in relation to position G38 and normalised by the corresponding precipitated pBpa-labelled Der1-Myc variant. The asterisk denotes a cross-reactivity of the anti-Myc antibody.c, Pulse chase assay to analyse the activity of pBpa-modified Der1 variants in the degradation of CPY*. The selected Der1 constructs (derived from pMM063) form prominent crosslinks with different components of the HRD ligase as well as CPY*-HA and were expressed on highcopy plasmids in $\Delta$ der 1 cells. As a control unlabelled Der1 was expressed on a low-copy (wt) and high-copy plasmid (Der1 OE), respectively.d, $\Delta$ der1 Ubc7C/S CPY*-HA cells expressing either various pBpa-labelled Der1-Myc constructs or unlabelled Der1-Myc were lysed in Digitonin containing buffer and subjected to immunoprecipitation with anti-Myc antibodies. Interaction partners of Der1-Myc were analysed by SDS-PAGE and immunoblotting. The asterisk denotes a cross-reactivity of the anti-Hrd1 antibody in the total lysate.

\section{Supplementary Figure 4}

Der1 is in close proximity to dislocated CPY*.

a, Photoreactive probes were placed at the indicated positions in Der1-Myc. The constructs were expressed in $\Delta d e r 1 \mathrm{Ubc7C/S} \mathrm{CPY}^{*}$-HA cells either containing or lacking Usa1 and exposed to UV light. The samples were then lysed and subjected to immunoprecipitation as described in Figure 3. b, Der1-HA expressed from high-copy plasmid pMM079 was transformed into $\Delta$ der1 Ubc7C/S cells either containing or lacking Usa1. Der1-Myc labelled with pBpa at position G38 (derived from pMM075) was co-expressed where indicated. Cells were lysed in Digitonin containing buffer and Der1-Myc was immunoprecipitated with antiMyc antibodies. Interacting Der1-HA was detected by immunoblotting. c, $\Delta$ der1 Ubc7C/S 
cells were transformed with a low-copy plasmid encoding Der1-HA. Microsomes of these cells were solubilised with NP40 and Der1-HA was precipitated with anti-HA antibodies. The catalytically inactive Ubc7 mutant (Ubc7C/S) was used to adjust the substrate levels in the individual strains. d, Der1-Myc constructs with photoreactive probes placed at positions which reveal prominent crosslinks with Hrd1 were expressed either in $\triangle$ der1 CPY*-HA cells (wt) or in $\triangle$ der1 $\mathrm{CPY}^{*}$-HA Ubc7C/S cells. The photocrosslinking was performed as described. $\mathbf{e}$, As in $\mathbf{d}$ but the Der1-Myc constructs contained photoreactive probes at positions, which formed crosslinks with Usa1. f, Determination of the unfolded protein response (UPR) in strains used for the crosslinking experiments by ß-galactosidase activity assay. The indicated yeast strains were transformed with the pUPRE-lacZ plasmid and the activity of $B$ galactosidase was measured as described (see Methods). Where indicated cells were treated with $4 \mathrm{mM}$ Dithiotriol (DTT) for 1 hour before ß-galactosidase measurement to fully induce the UPR. Error bars and mean values of three independent experiments are shown. g, Der1-Myc variants labelled at the indicated positions were expressed in $\Delta p e p 4 \mathrm{Ubc} 7 \mathrm{C} / \mathrm{S}$ cells containing plasmid-encoded PrA*-HA. The crosslinking experiment was performed as in a.

\section{Supplementary Figure 5}

The pBpa-labelled Der1RN-Myc transmembrane mutant is properly assembled with the HRD-ligase but displays alterations in the crosslinking to its interaction partners.

a, $\Delta$ der1 Ubc7C/S CPY*-HA cells were transformed with high-copy plasmids encoding either pBpa-modified Der1-Myc (derived from pMM075), Der1RN-Myc (derived from pMM074) or unlabelled Der1-Myc (pMM075). Digitonin-solubilised membranes of the total extract were subjected to immunoprecipitation with anti-Myc antibodies (left and right panel). b, As in a but the microsomes were solubilised with NP40 before precipitation of the Der1-Myc constructs.

\section{Supplementary Figure 6}

Full gel scans of key blots shown in the main figures of this manuscript. Antibodies used for protein detection are indicated.

\section{Supplementary Table 1}

Characterisation of Der1 mutants generated by site-directed mutagenesis.

The indicated amino acids in Der1 were changed to alanine $(A)$, leucine $(L)$ or tryptophan $(W)$ and analysed for their stability and their ability to promote $\mathrm{CPY}^{*}$ degradation by cycloheximide decay assay. The interaction to Usa1 and the ability to form oligomers were determined by immunoprecipitation. Der1 transmembrane mutants deficient for CPY* degradation and used for following experiments are highlighted in red. 


\section{Supplementary Table 2}

Yeast strains used in this study

\section{Supplementary Table 3}

Plasmid constructs used in this study 


\section{Supplementary Figure-1 (Jarosch)}

a

Derlin-1_hs Derlin-1_ce Derlin-3_hs Der7in-3_mm Derlin-2_hs Derlin-2_ce Der1_sc

Derlin-1_hs Der1in-1_ce Derlin-3_hs Derlin-3_mm Derlin-2_hs Der1in-2_ce Der1_sc

Derlin-1_hs Derlin-1_ce Derlin-3_hs Derlin-3_mm Derlin-2_hs Derlin-2_ce Der1_sc

Derlin-1_hs Derlin-1_ce Der1in-3_hs Derlin-3_mm Derlin-2_hs Derlin-2_ce Der1_sc

Der7in-1_hs Derlin-1_ce Derlin-3_hs Derlin-3_mm Derlin-2_hs Derlin-2_ce Der1_sc

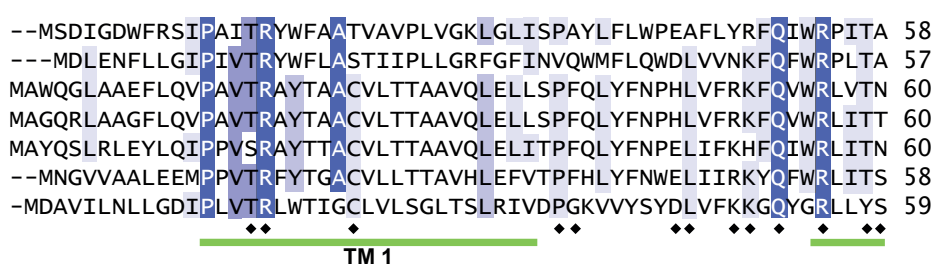

TFYFPVGPGTGFLYLVNLYFLYQYSTRLETGAFDGRPADYLFMLLFNWICIVITGLAMDM 118 LIYYPVTPQTGFHWLMMCYFLYNYSKALESETYRGRSADYLFMLIFNWFFCSGLCMALDI 117 FLFFGP---LGFSFFFNMLFVFRYCRMLEEGSFRGRTADFVFMFLFGGVLMTLLGLLGSL 117 FLFFGP---LGFGFFFNMLFVFRYCRMLEEGSFRGRKADFVFMFLFGGVLMTLLGFLGSL 117 FLFFGP---VGFNFLFNMIFLYRYCRMLEEGSFRGRTADFVFMFLFGGFLMTLFGLFVSL 117 FCFFGS---FGFSFLFNMIFTYRYCMMLEEGSFRGRRADFVYMFLFGAVLMILSGIFVQI 115 IFDYGA---FNWISMINIFVSANHLSTLEN-SFNLRRKFCWIIFLLLVILVKMTSIEQPA 115 TM $2 \bullet \bullet \bullet \bullet \bullet+\bullet$

QLLMIPLIMSVLYVWAQLN-RDMIVSFWFGTRFKACYLPWVILGFNYIIGGSVINELIG- 176 YFLLEPMVISVLYWWCQVN-KDTIVSFWFGMRFPARYLPWVLWGFNAVLRGGGTNELVG- 175 FFLGQALMAMLVYVWSRRS-PRVRVNFFGLLTFQAPFLPWALMGFSLLLGNSILVDLLG- 175 FFLGQALMAMLVYVWSRRS-PHVRVNFFGLLNFQAPFLPWALMGFSLLLGNSVVTDLLG- 175 VFLGQAFTIMLVYVWSRRN-PYVRMNFFGLLNFQAPFLPWVLMGFSLLLGNSIIVDLLG- 175 LFLGQAFTIMLVYIWSRRN-PMIQMNFFGVLTFTAPYLPWVLLLFSLLLGNNAVVDFMG- 173 ASLGVLLHENLVYYELKKNGNQMNVRFFGAIDVSPSIFPIYMNAVMYFVYKRSWLEIAMN 175 $-$ TM 4

NLVGHLYFFLMFRYPMDLGGRNFLSTPQFLYRWLPSRRGGVSGFGVPPASMRRAADQNGG 236 ILVGHAYFFVALKYPDEYG-VDLISTPEFLHRLIPDEDGGIHGQ----DGNIRGARQQPR 230 IAVGHIYYFLEDVFPNQPGGKRLLQTPGFLGLQSSKAPAGSSLT---.---IWTQQSQG 227 ILVGHIYYFLEDVFPNOPGGKRLLLTPSVLKLLLDDPOEDPDYL--------PLPEEQP- 226 IAVGHIYFFLEDVFPNQPGGIRILKTPSILKAIFDTPDEDPNYN-------PLPEERPG 227 IACGHIYFFLEDVFPFQEHGKRFLKTPQWLVYLFDERRPEP----_-----LPEDERPG 222 FMPGHVIYYMDDIIGKIYG-IDLCKSPYDWFRNTETP---.--

GGRHNWGQGFRLGDQ 251 GHQWPGGVGARLGGN 245 GPGTAGELAAPS--- 239 -----EL------ 228 GFAWGEGQRLGG--- 239 GFEWGDEQPEQEQHD 237 --.---.---.-- b

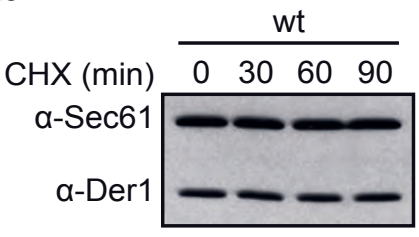

$\Delta$ usa1 $\Delta h r d 1$

$\mathrm{CH}$
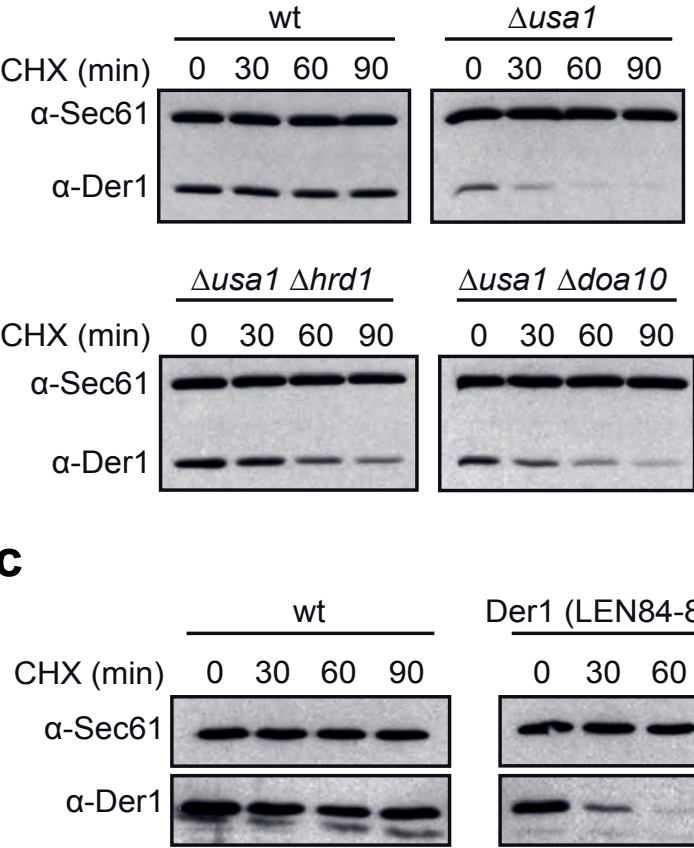

$\operatorname{Sec} 61$

Der1

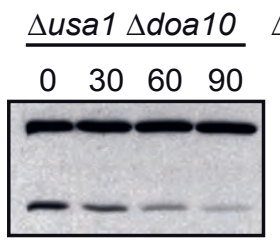

$\Delta u s a 1 \Delta h r d 1 \Delta d o a 10$

$\begin{array}{llll}0 & 30 & 60 & 90\end{array}$

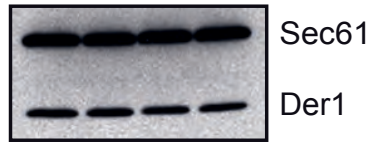

Der1 (LEN84-86AAA)

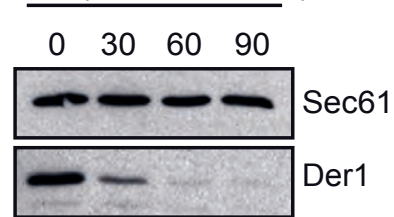

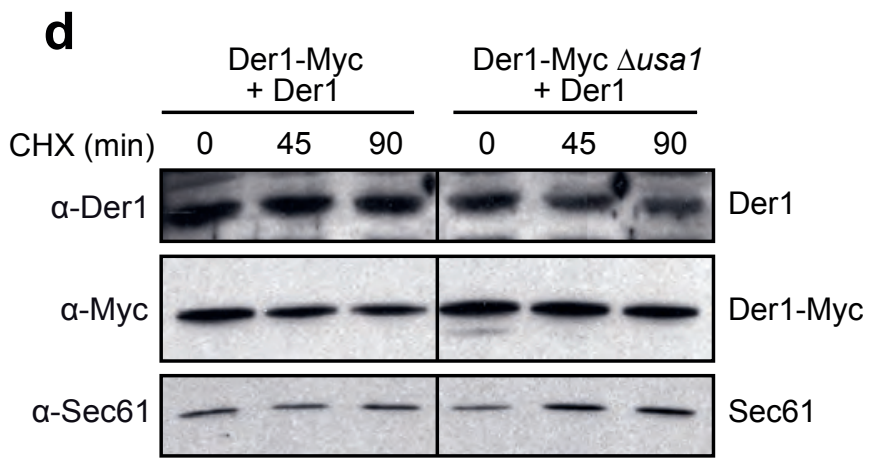

e

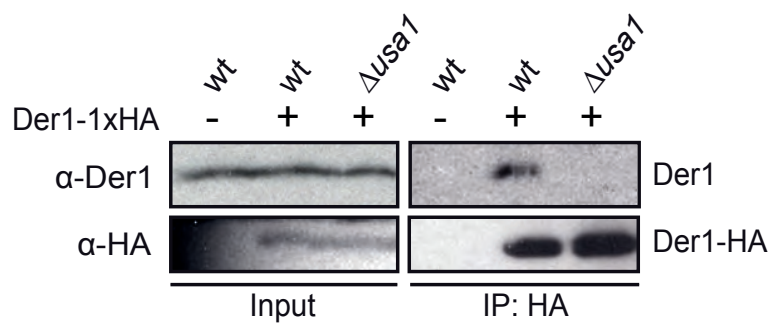

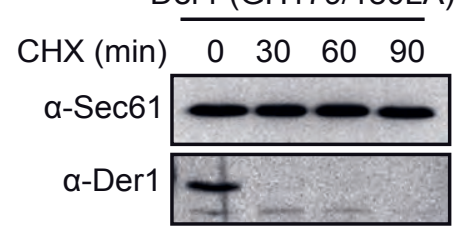

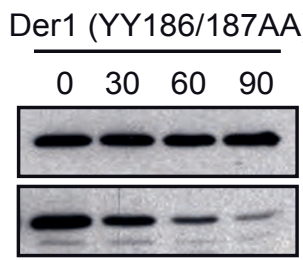

Der1 (P201A)

$\begin{array}{llll}0 & 30 & 60 & 90\end{array}$

$\operatorname{Sec} 61$

Der1 
Supplementary Figure-2 (Jarosch)

a

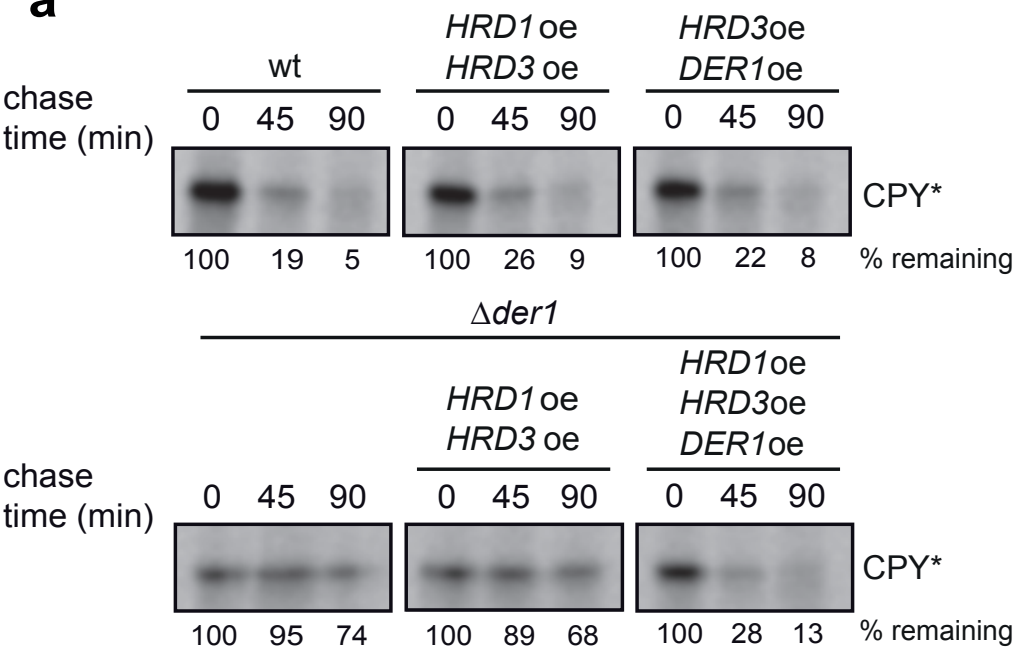

HRD1oe

$D E R 10 \mathrm{e}$
$0 \quad 45 \quad 90$

b
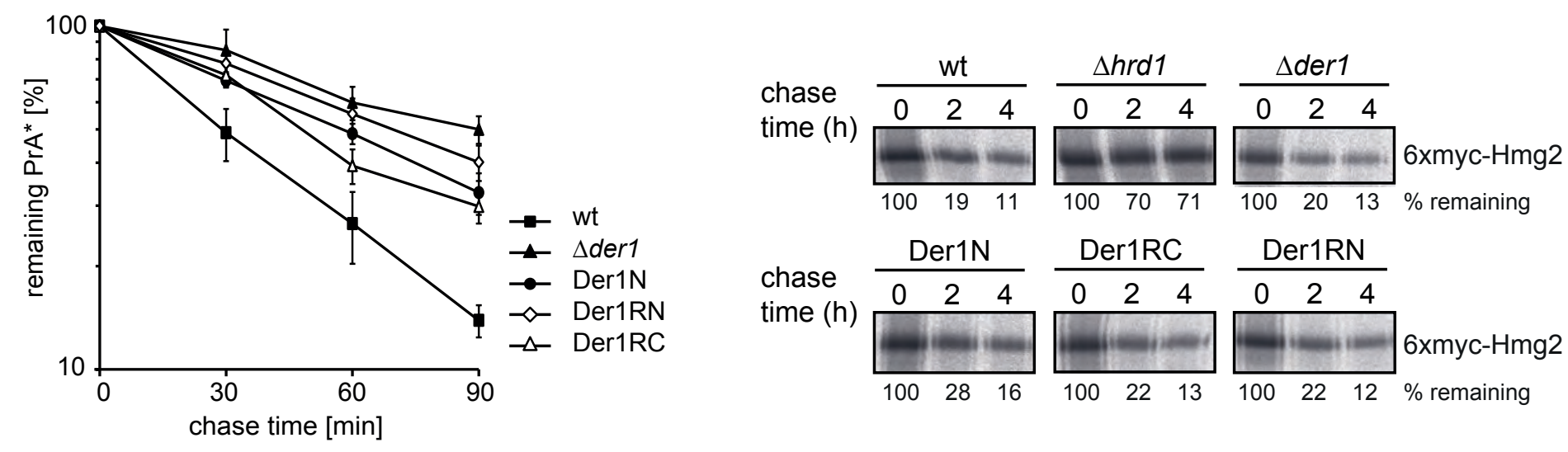

C

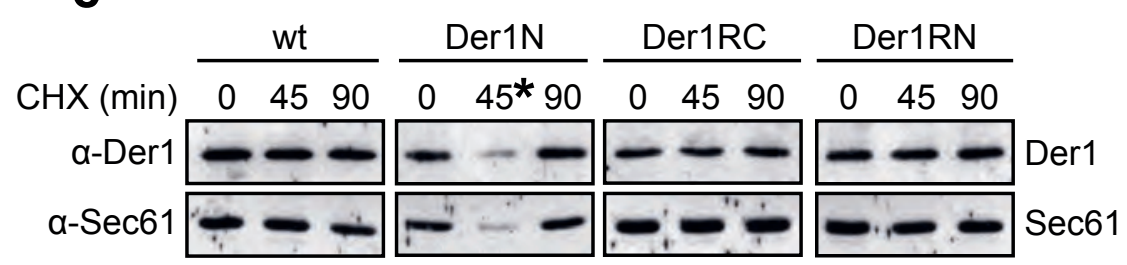

d

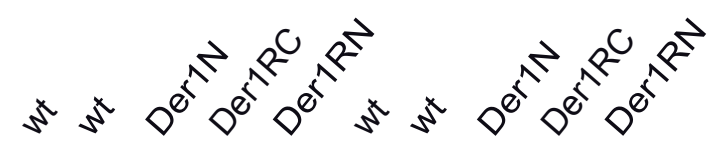

Usa1-Myс - $++++\quad+\quad+\quad+++$

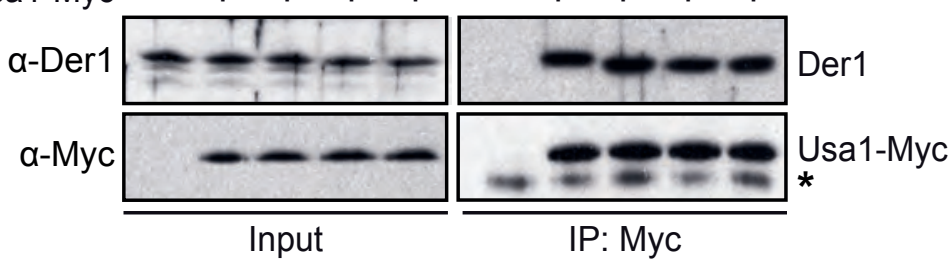

e
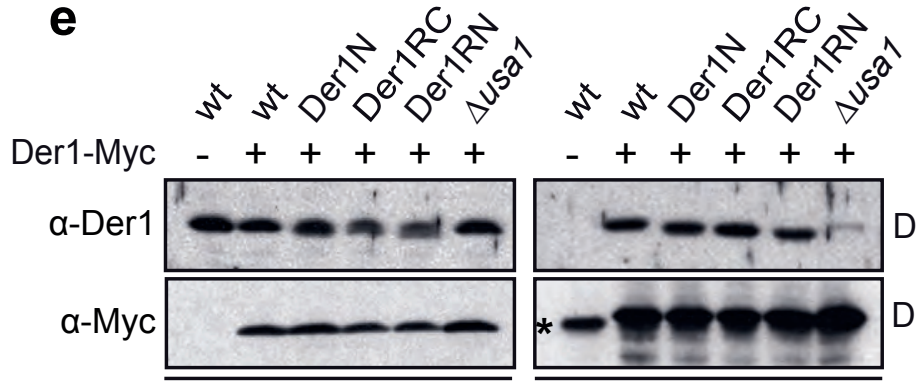

Der1

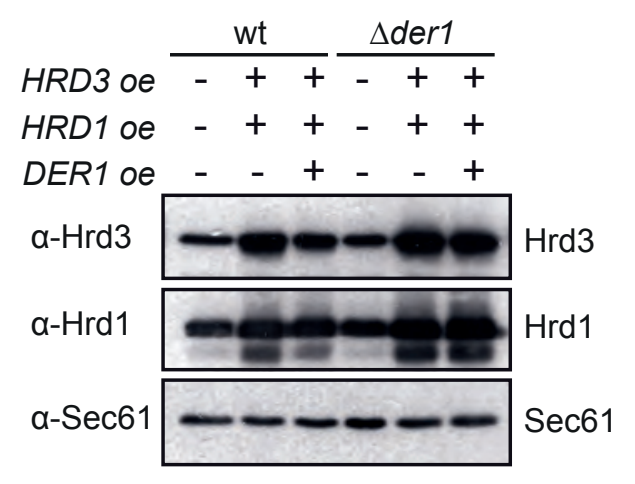




\section{Supplementary Figure-3 (Jarosch)}

a

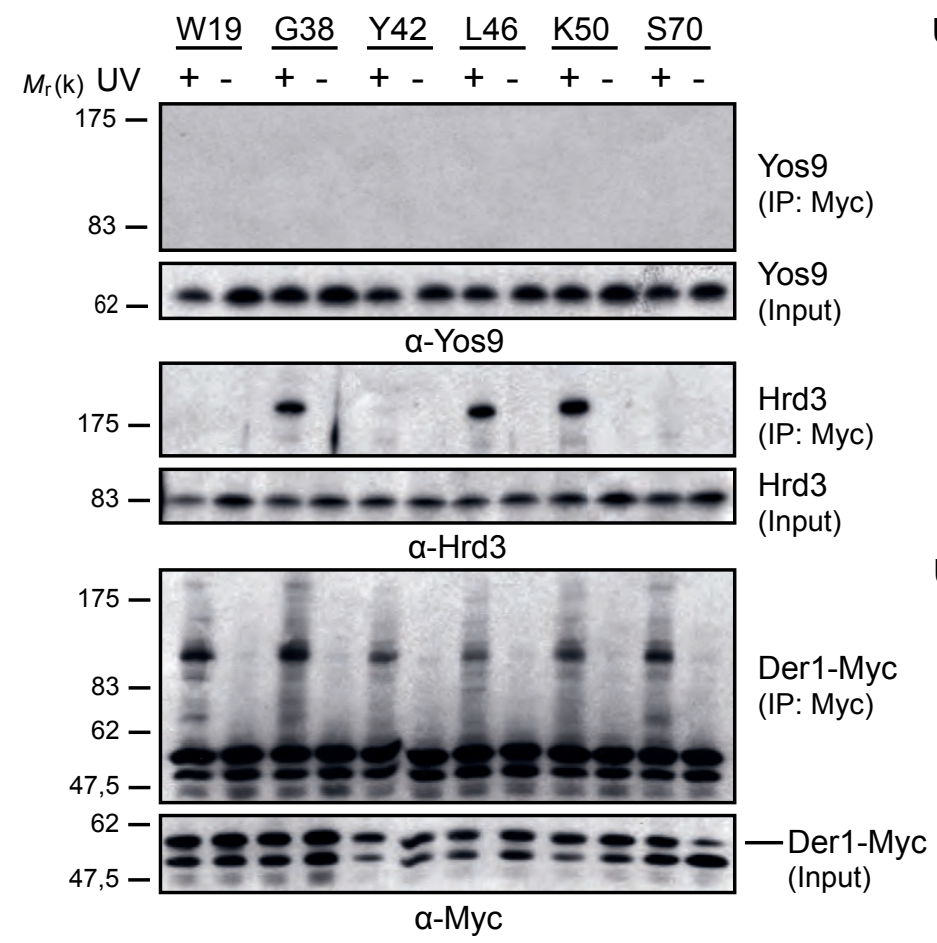

b

UV $\frac{\text { W19 }}{+-} \frac{\mathrm{S} 31}{+-} \frac{\mathrm{R} 33}{+-} \frac{\mathrm{G} 38}{+-} \frac{\mathrm{L} 46}{+-} \frac{\mathrm{K} 50}{+-}$

$\begin{array}{lllllll}34 & 45 & 64 & 100 & 63 & 56 & \begin{array}{c}\% \text { crosslinked CPY*-HA relative to G38 } \\ \text { (normalised by precipitated Der1-Myc) }\end{array}\end{array}$

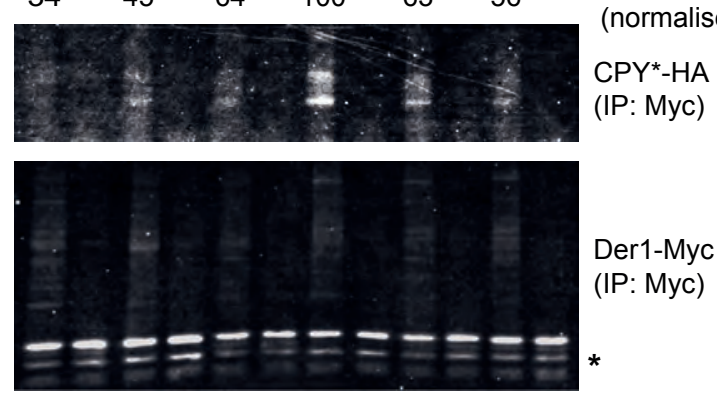

$\frac{\mathrm{Y} 58}{+-} \frac{\mathrm{I} 60}{+-} \frac{\mathrm{D} 62}{+-} \frac{\mathrm{F} 66}{+-} \frac{\mathrm{I} 146}{+-}$

$\begin{array}{lllll}16 & 40 & 48 & 26 & 52 \quad \% \text { crosslinked } \mathrm{CPY}^{*}-\mathrm{HA} \text { relative to G38 }\end{array}$
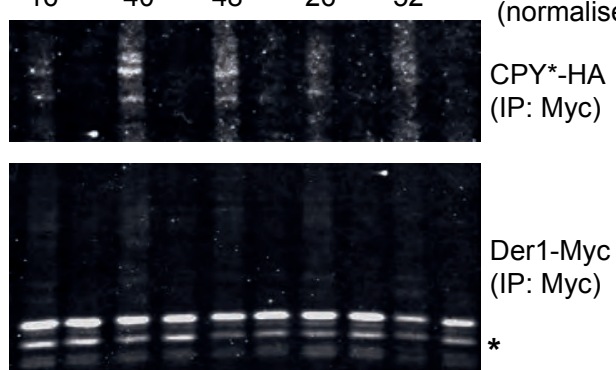

C

$\Delta d e r 1$

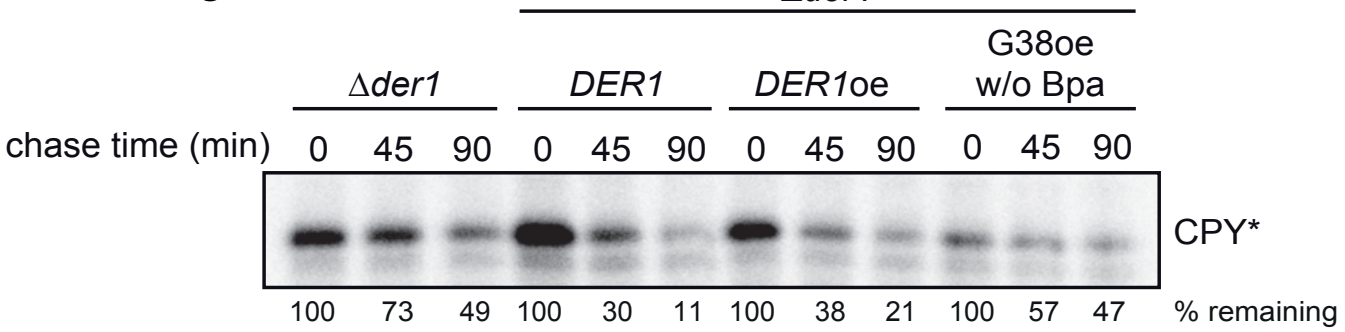
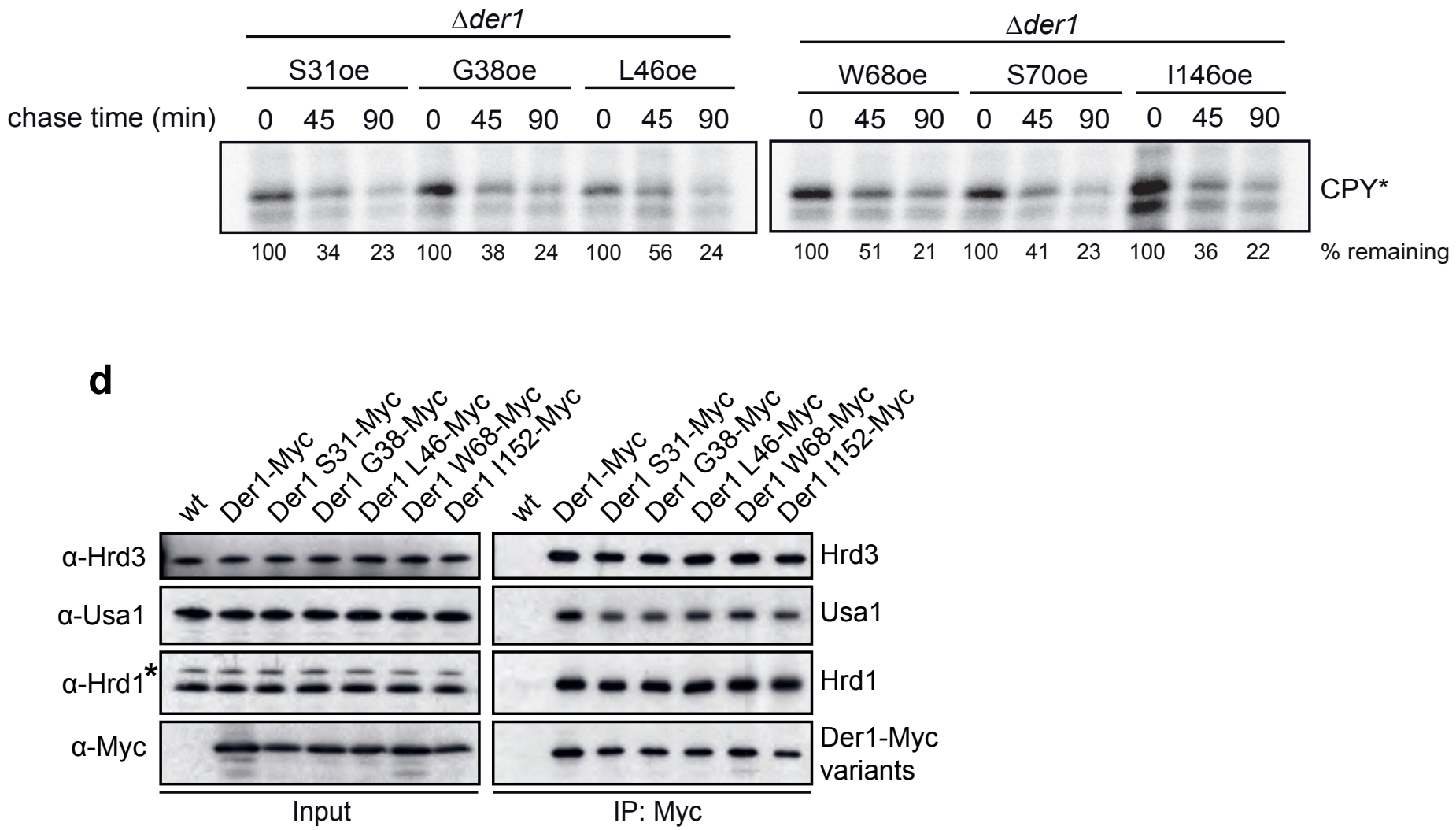


\section{Supplementary Figure-4 (Jarosch)}

a

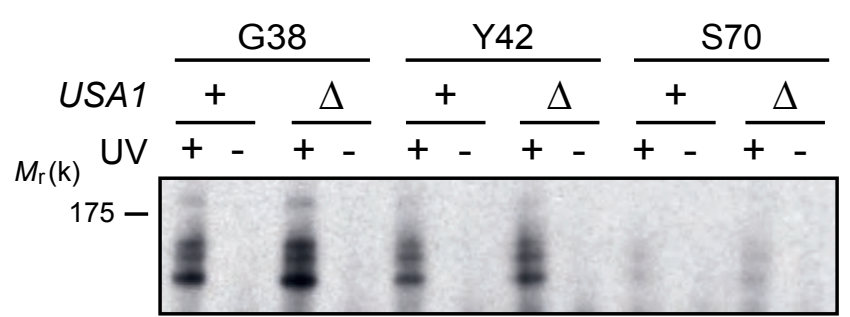

CPY*-HA (IP: Myc)

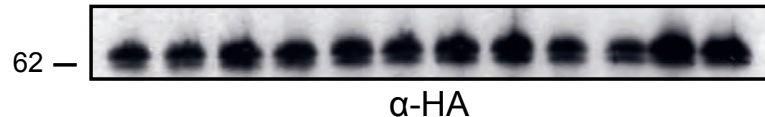
$-\mathrm{CPY}^{*}-\mathrm{HA}$

(Input)

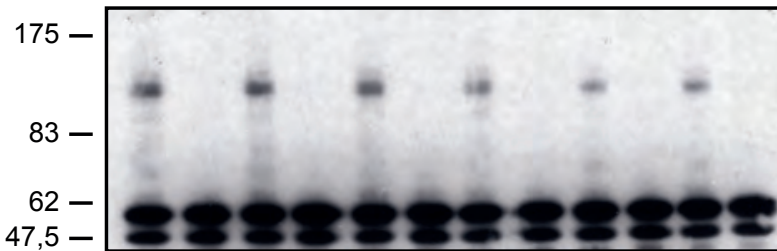

Der1-Myc (IP: Myc)

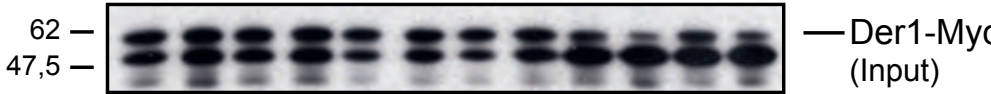

b

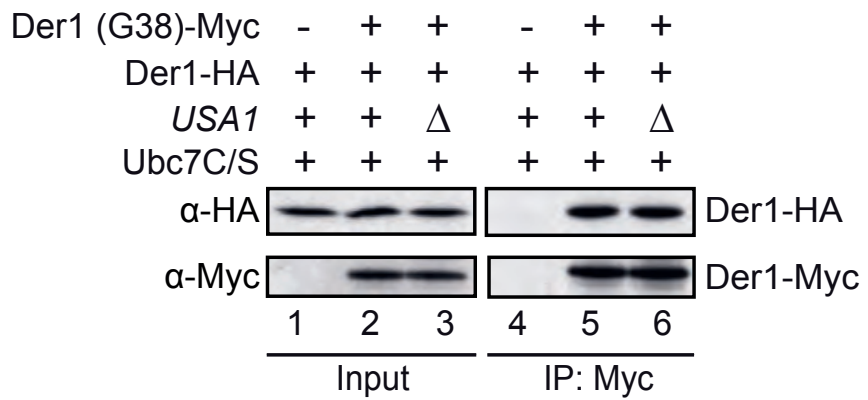

C

Der1-HA - $+\quad+\quad-\quad+\quad+$

USA1 $++\Delta++\Delta$

$\mathrm{Ubc7C} / \mathrm{S}++++++$

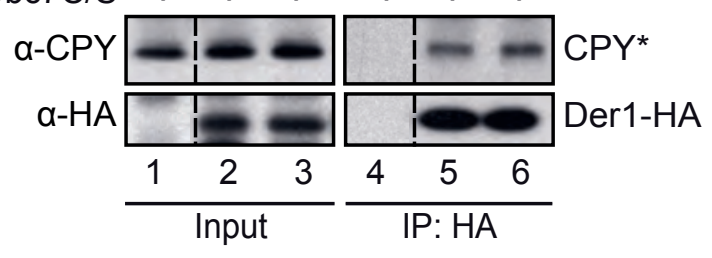

d

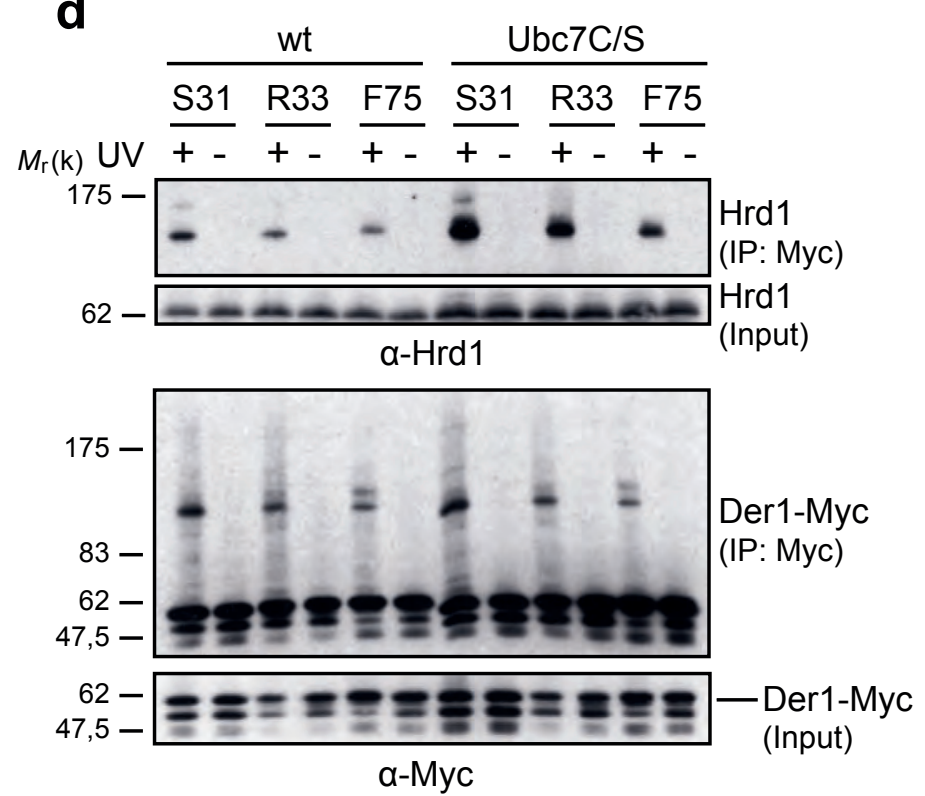

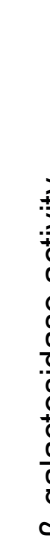

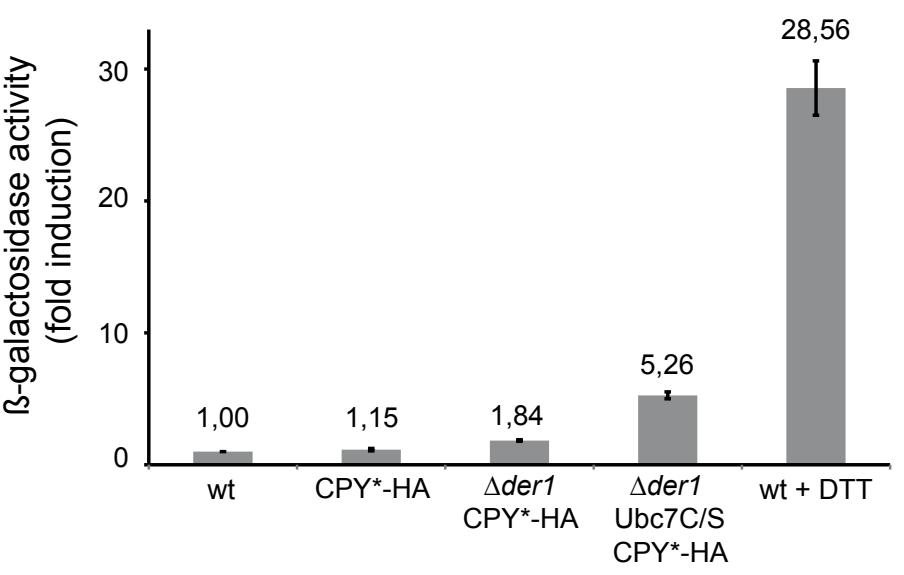

e

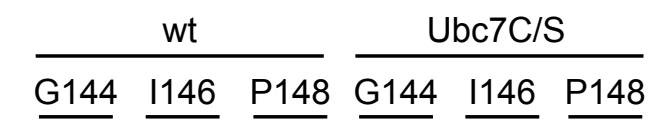

UV + t + t $\quad+$ t + +

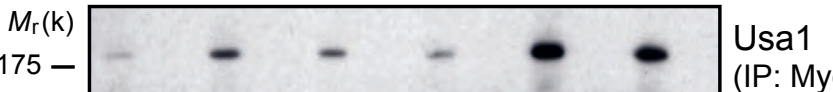

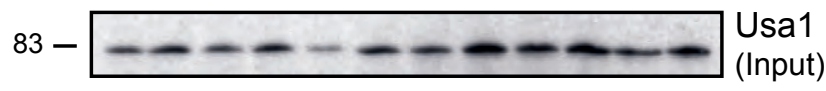
a-Usa1

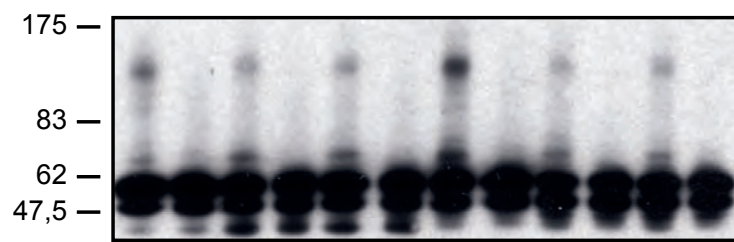

Der1-Myc (IP: Myc)

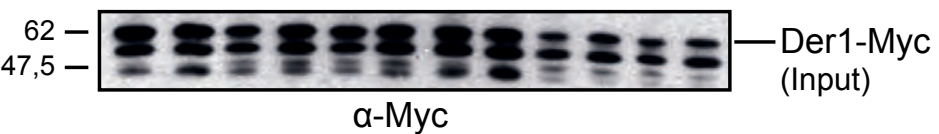

g
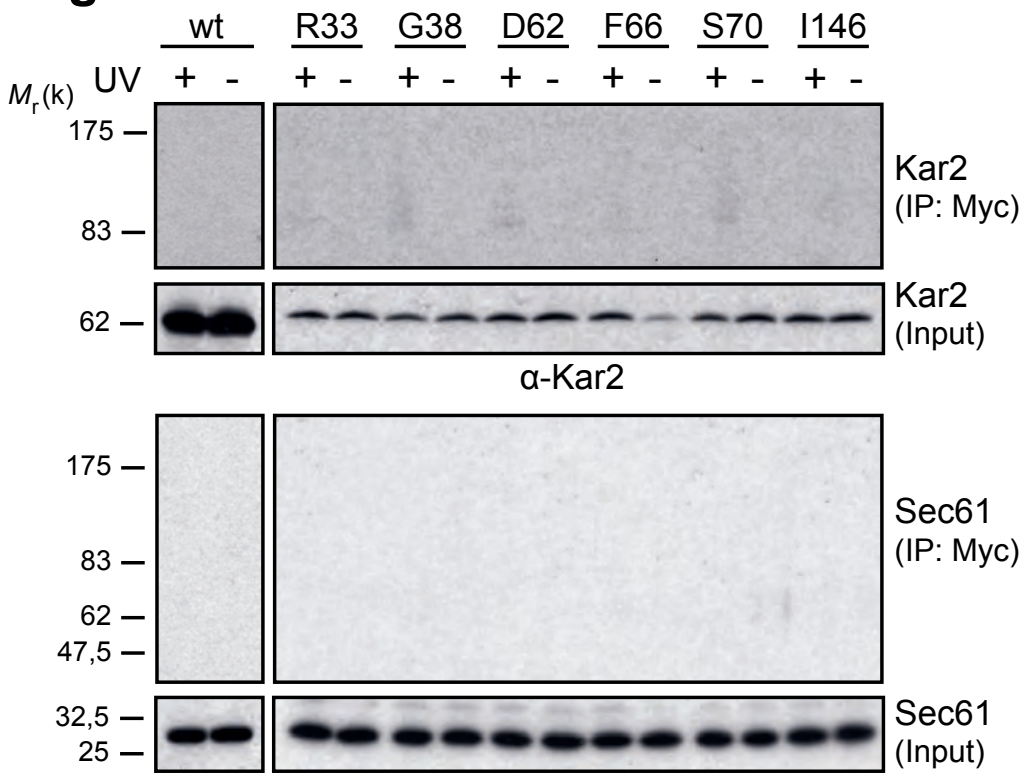

a-Kar2

Sec61 (IP: Myc) 


\section{Supplementary Figure-5 (Jarosch)}
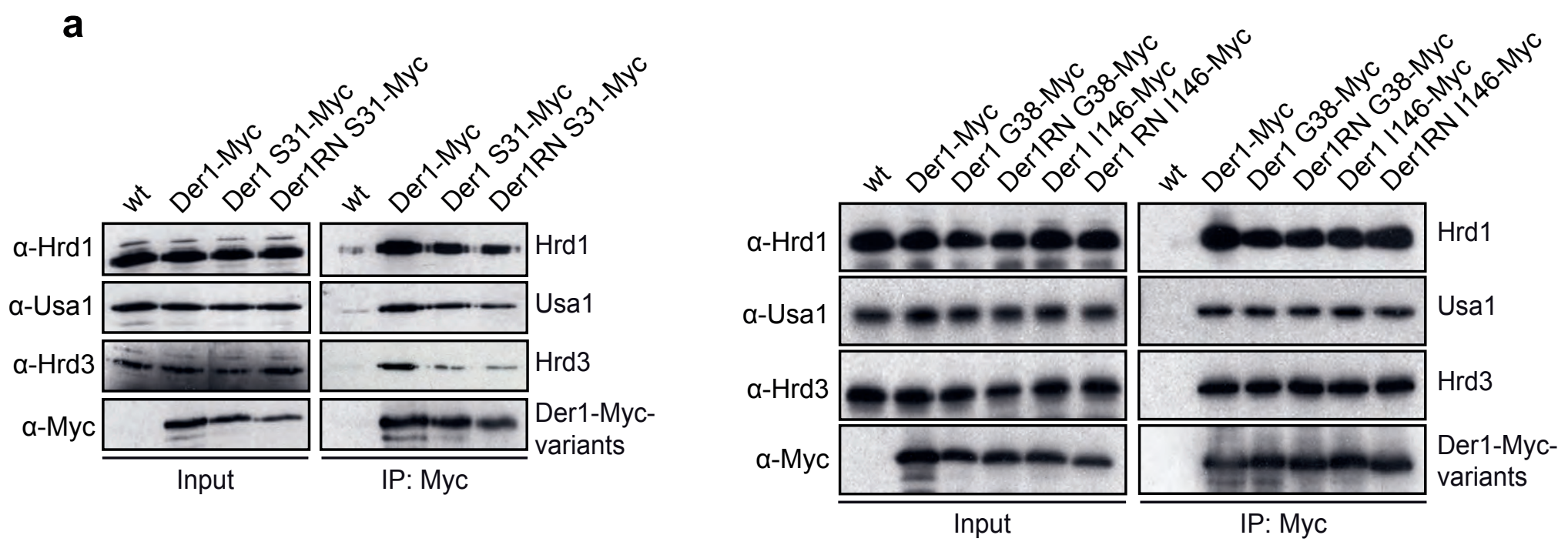

b

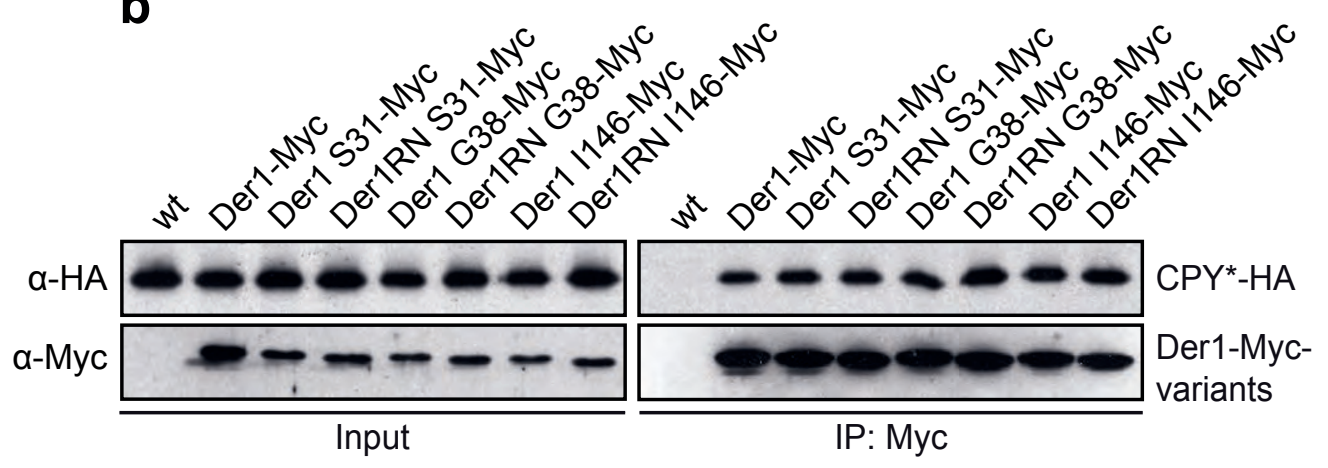


Supplementary Figure-6 (Jarosch)

Fig. 1a
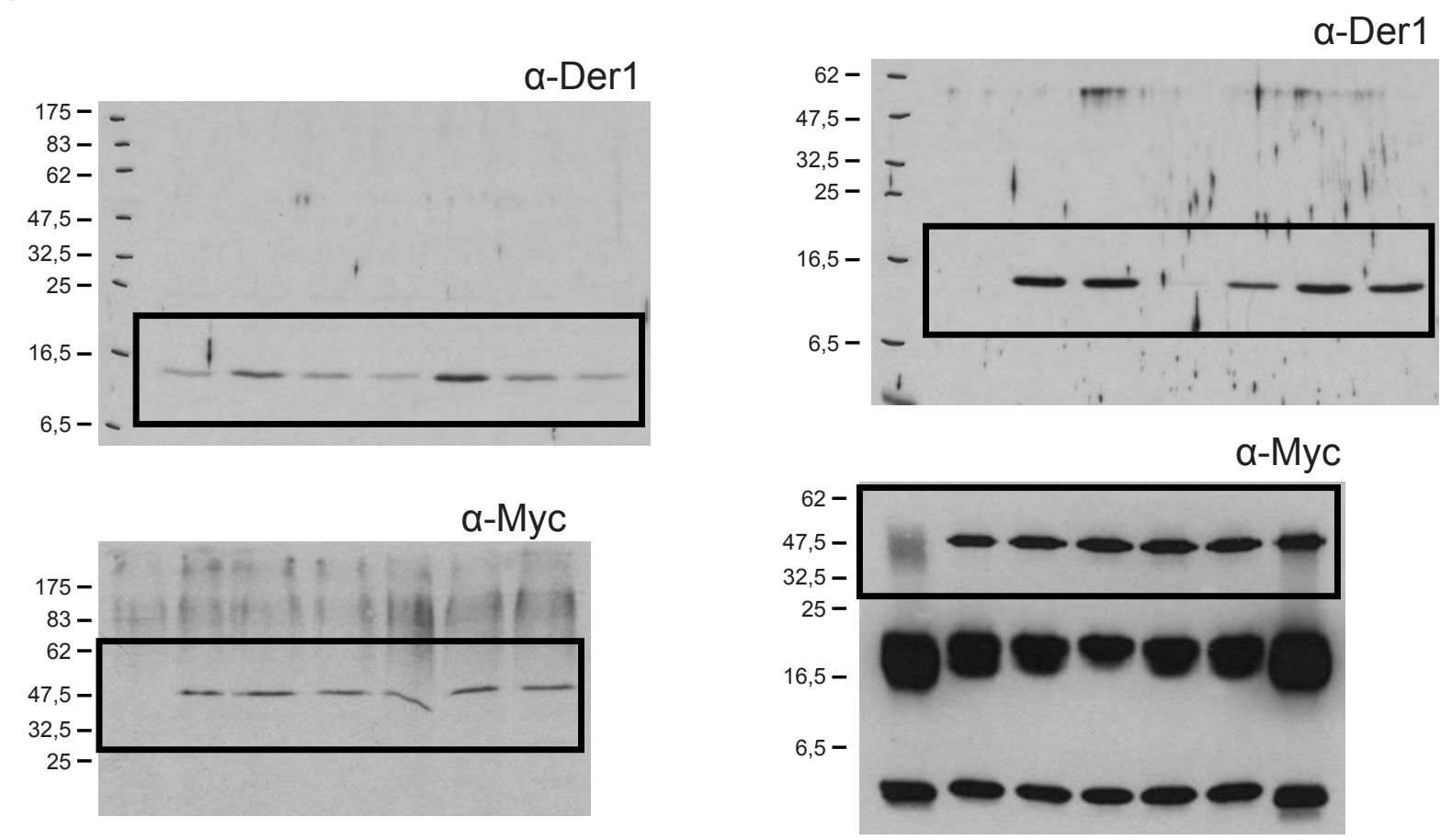

Fig. 1b
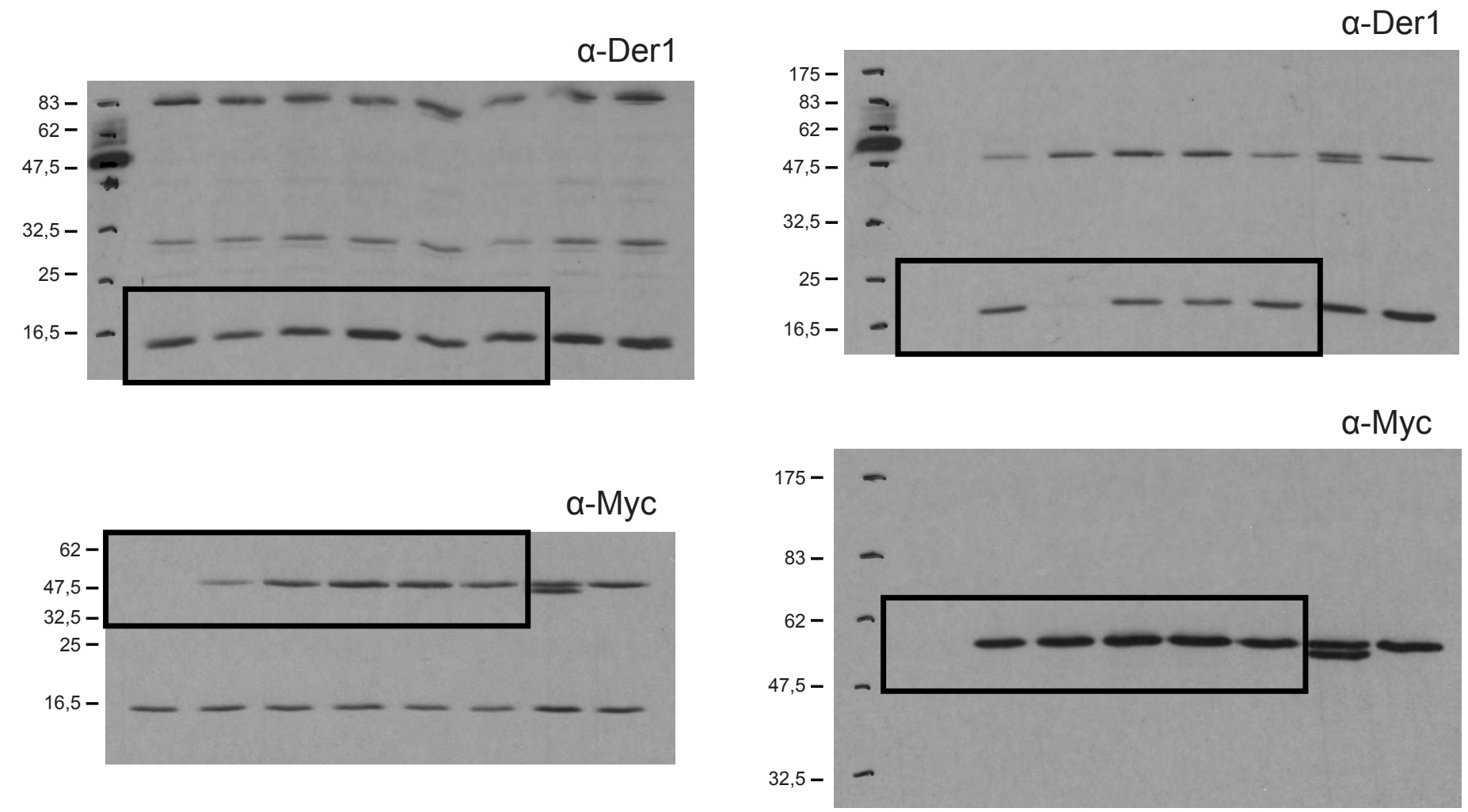
Supplementary Figure-6 (Jarosch) continued

Fig. $2 e$
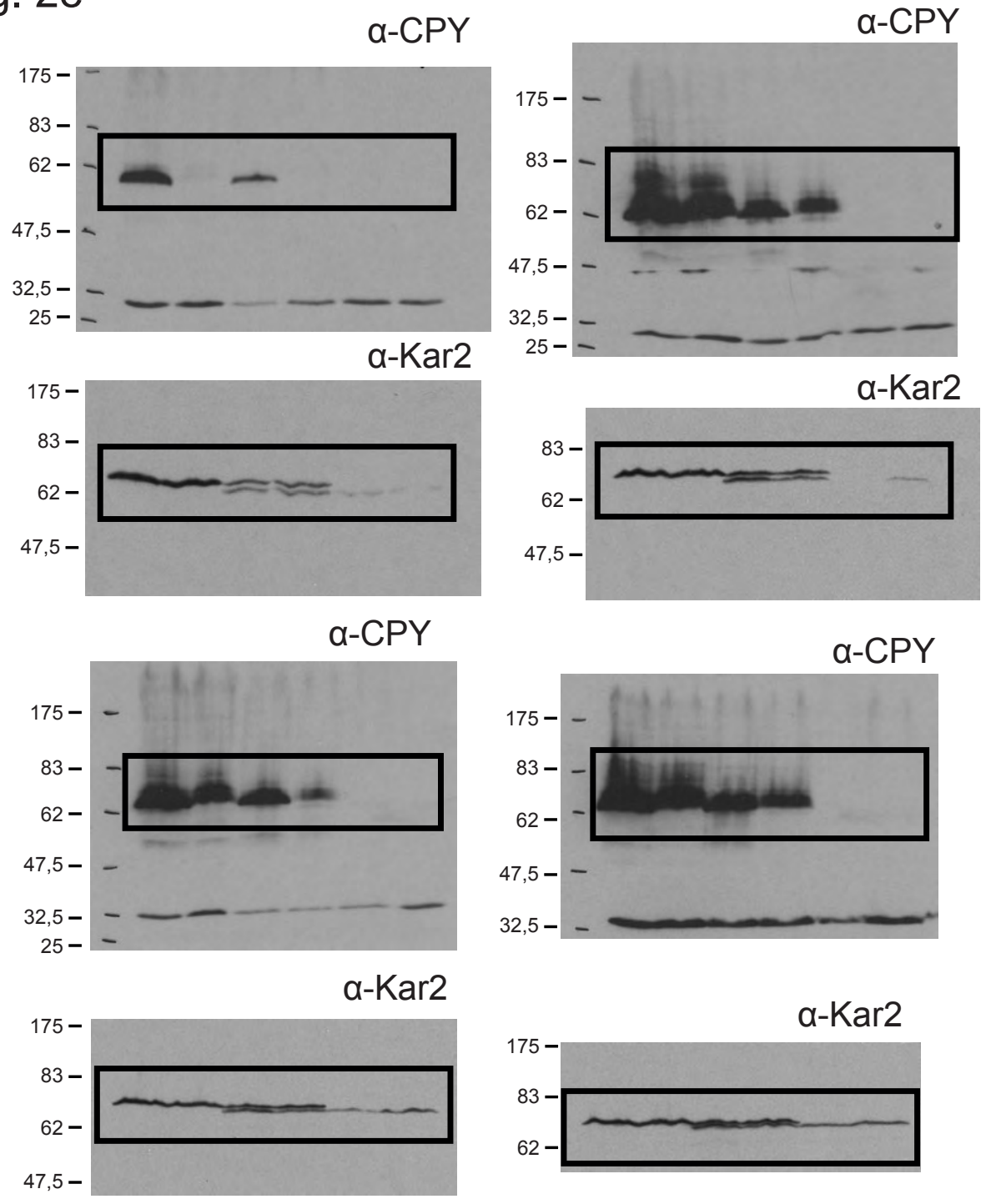
Supplementary Figure-6 (Jarosch) continued
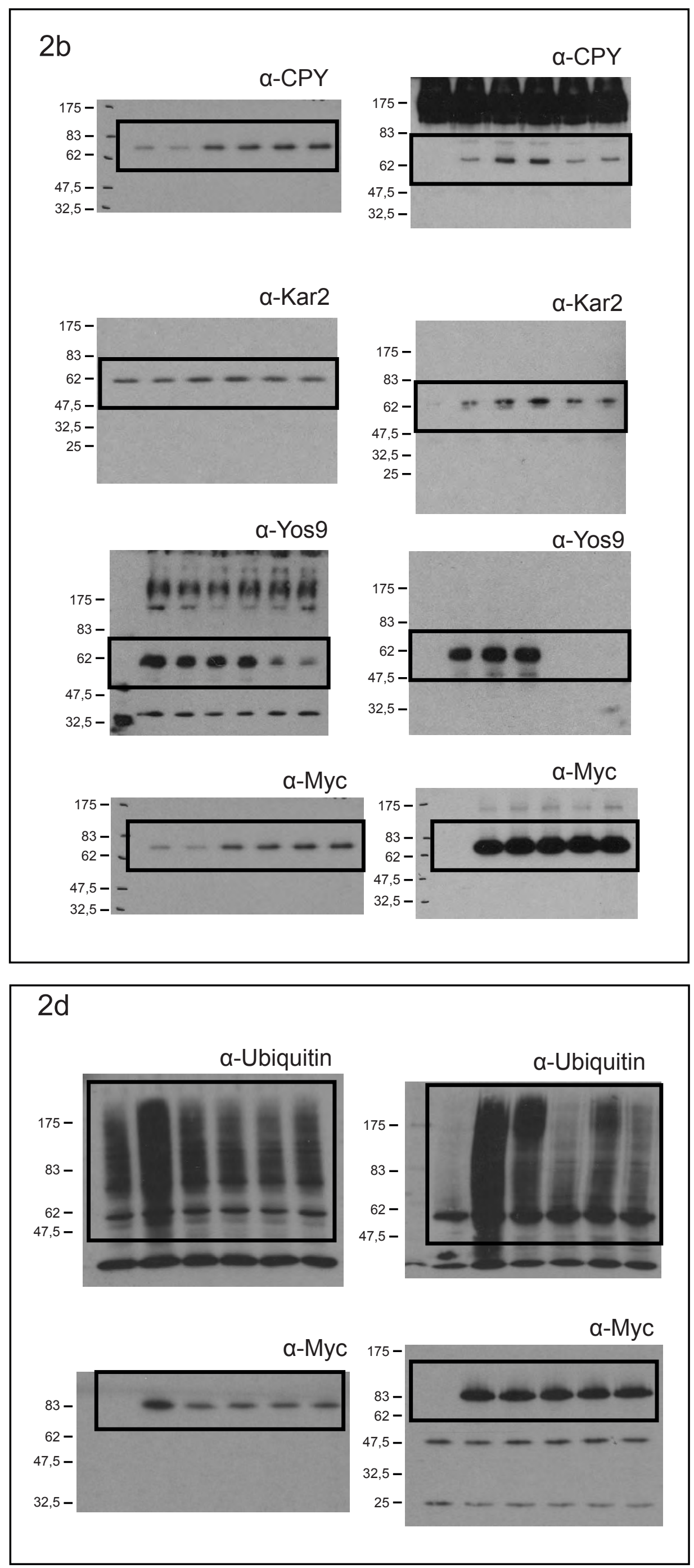
Supplementary Figure-6 (Jarosch) continued

Fig. 3
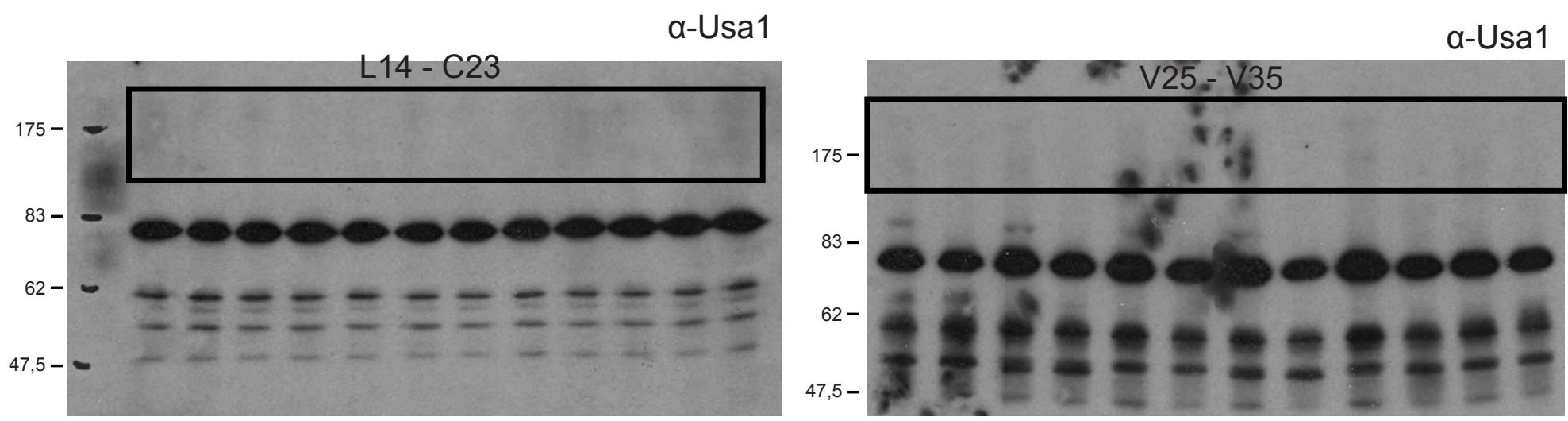

a-Usa1

wt

$\mathrm{G} 38-\mathrm{Y} 44$

F48

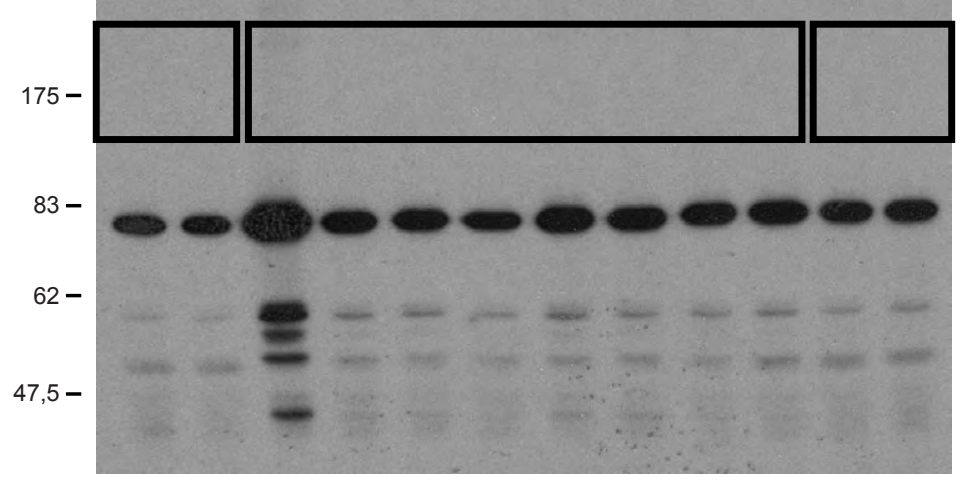

a-Usa1
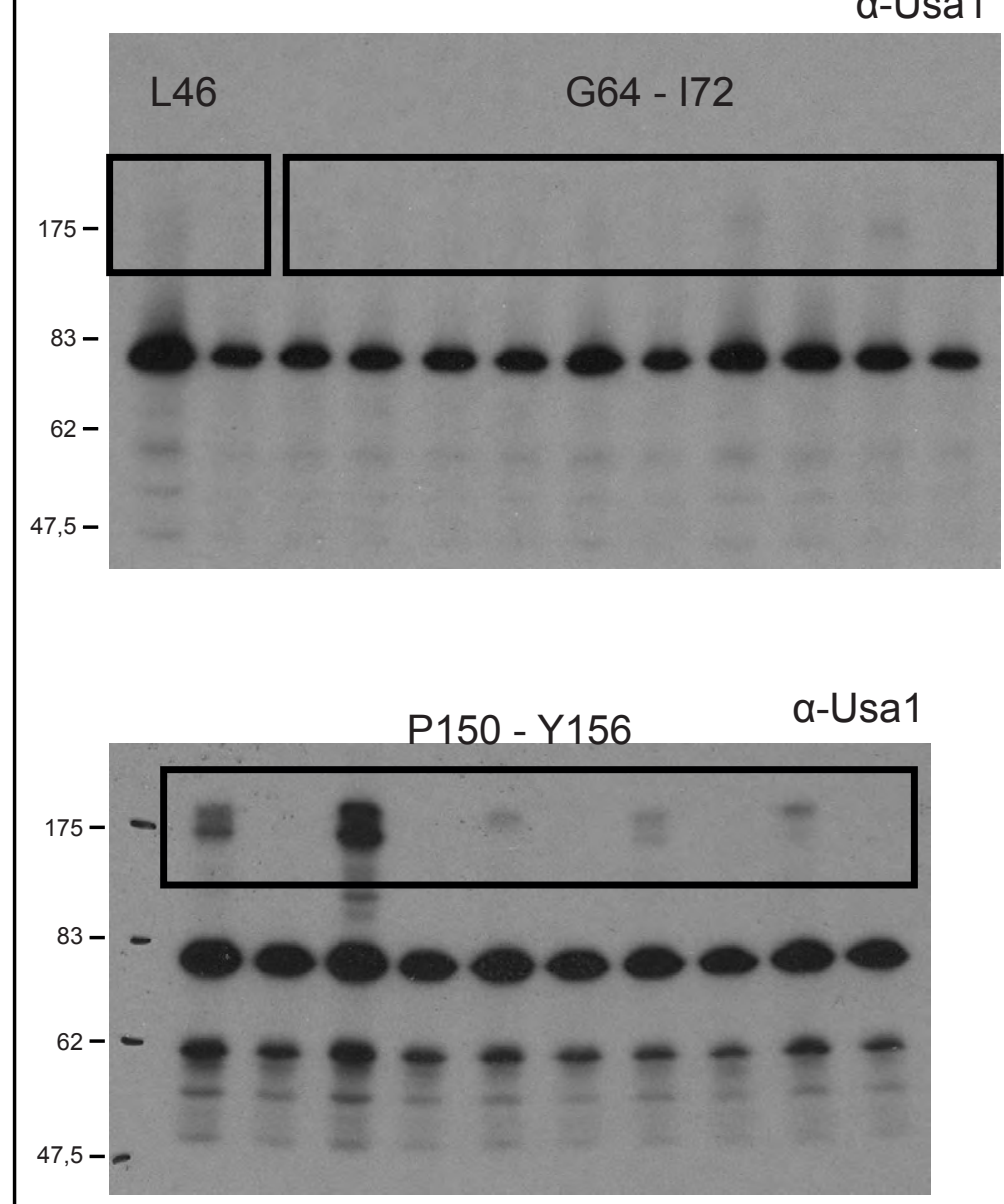

$\alpha-U s a 1$
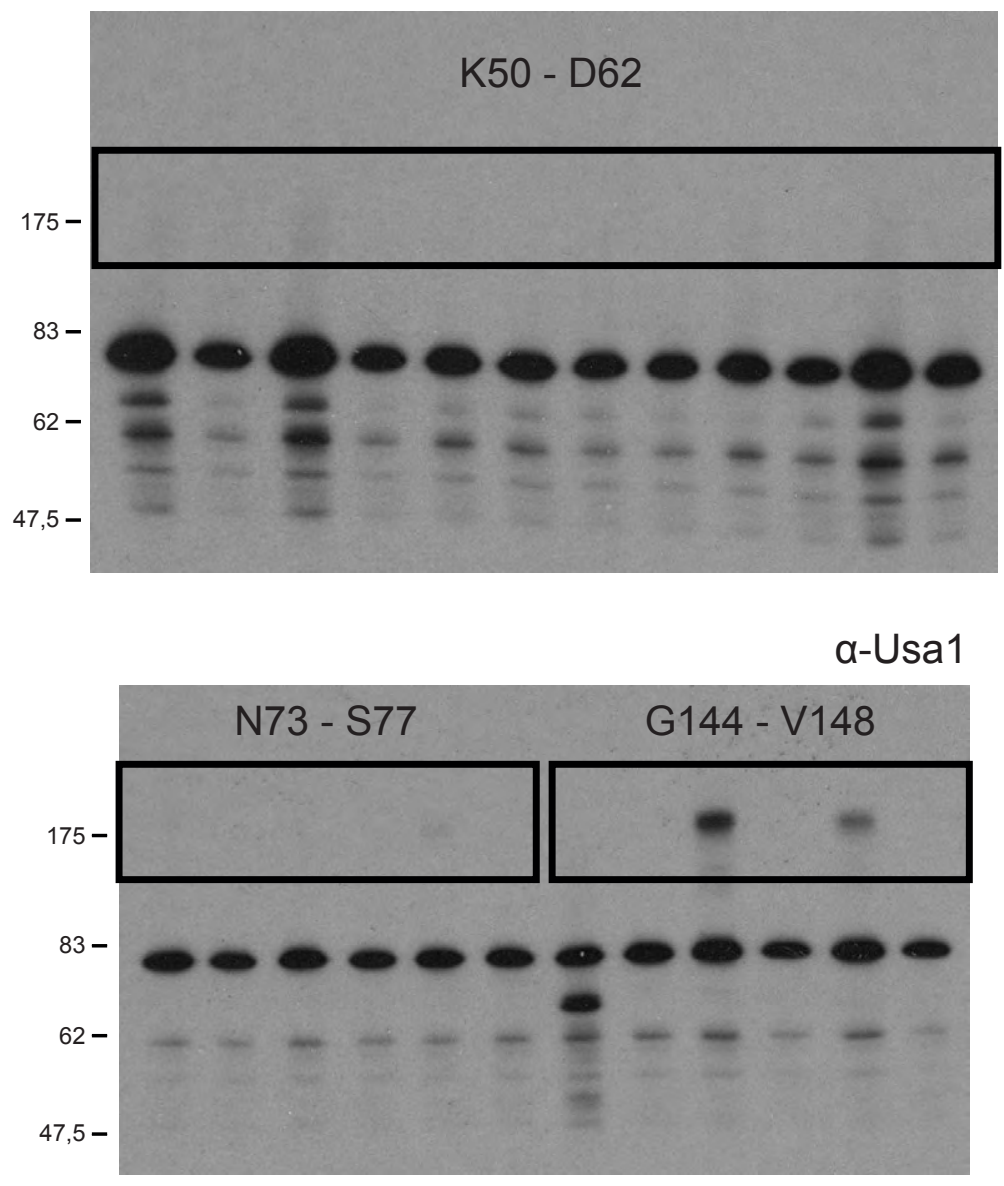

a-Usa1

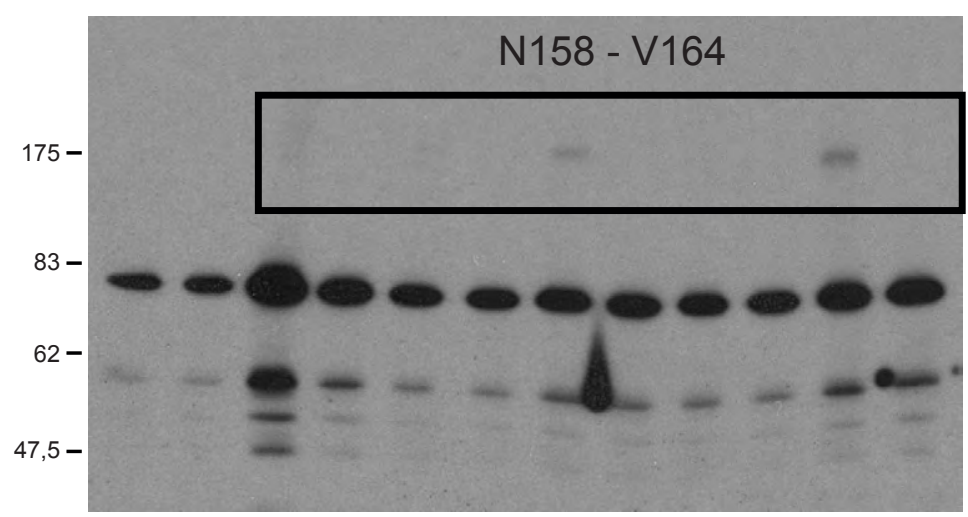


Supplementary Figure-6 (Jarosch) continued

Fig. 3
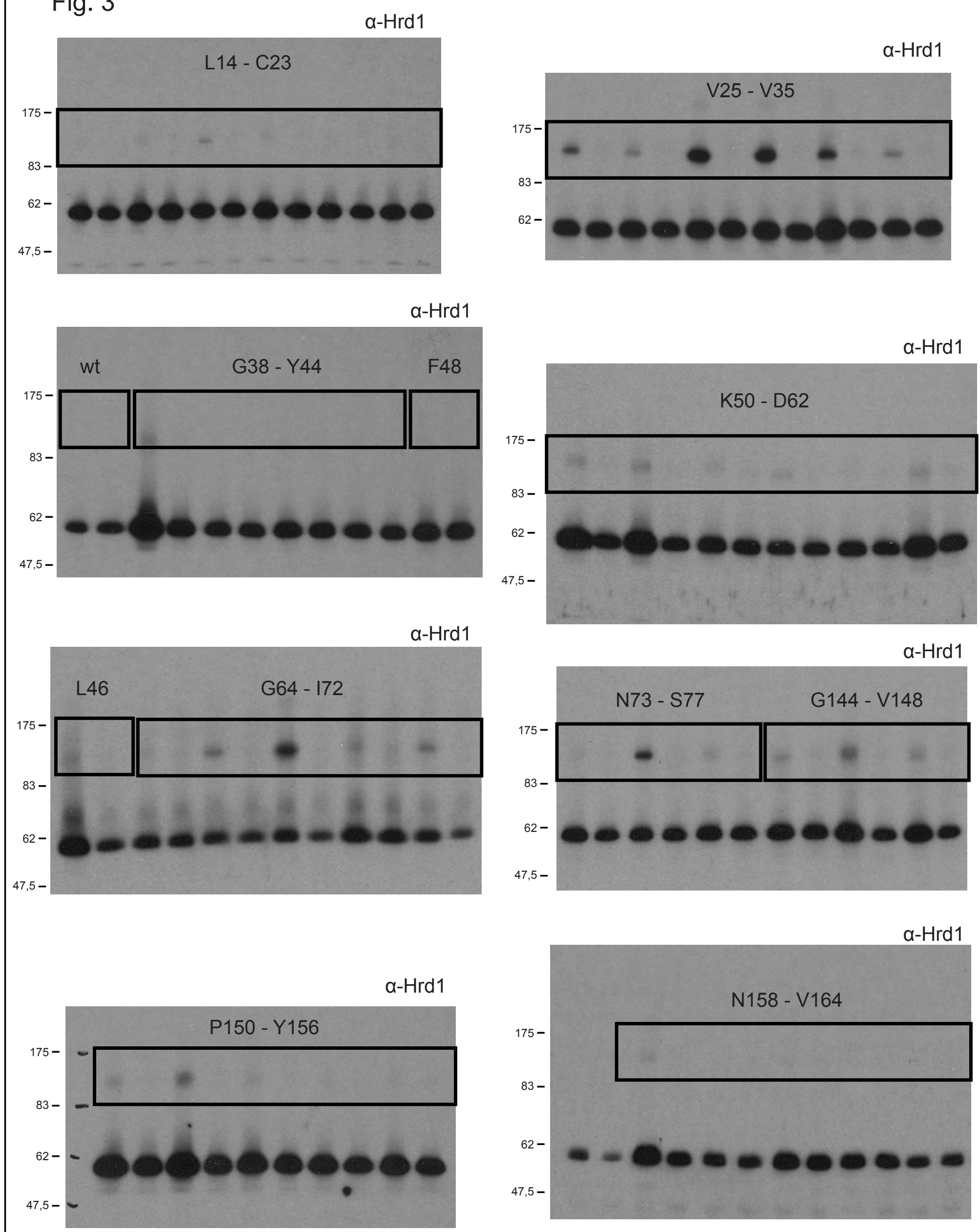

$\alpha-\mathrm{Hrd1}$

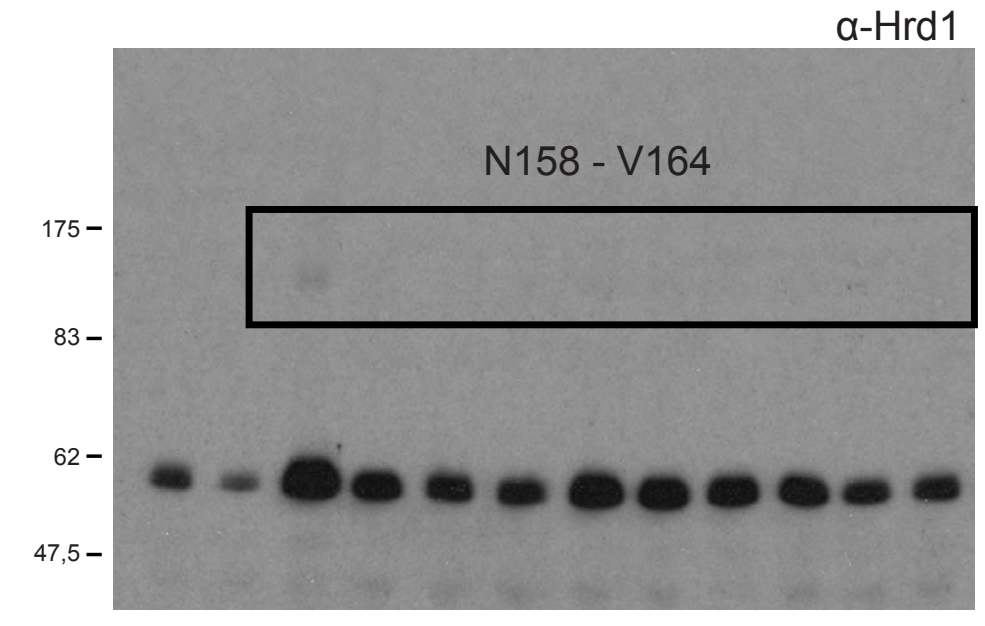


Supplementary Figure-6 (Jarosch) continued

Fig. 3

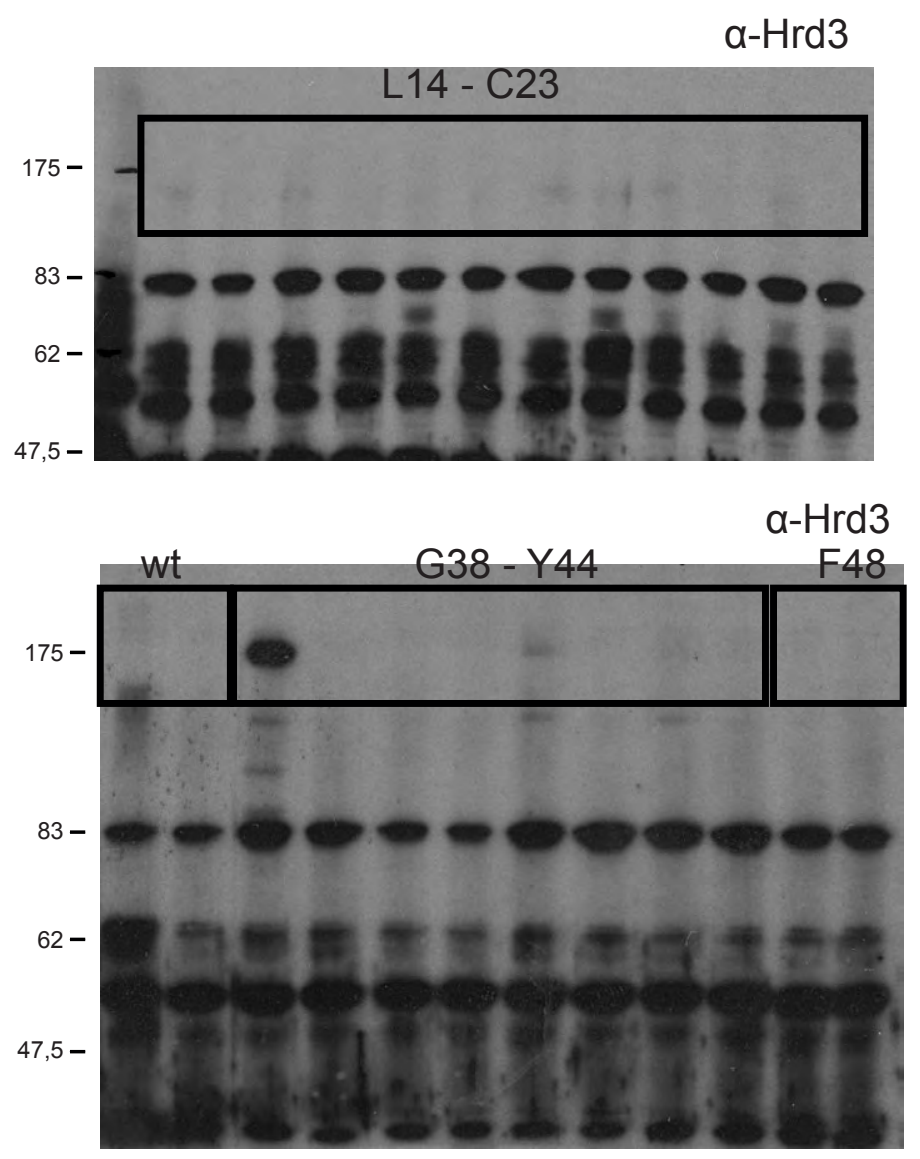

$\alpha-H r d 3$

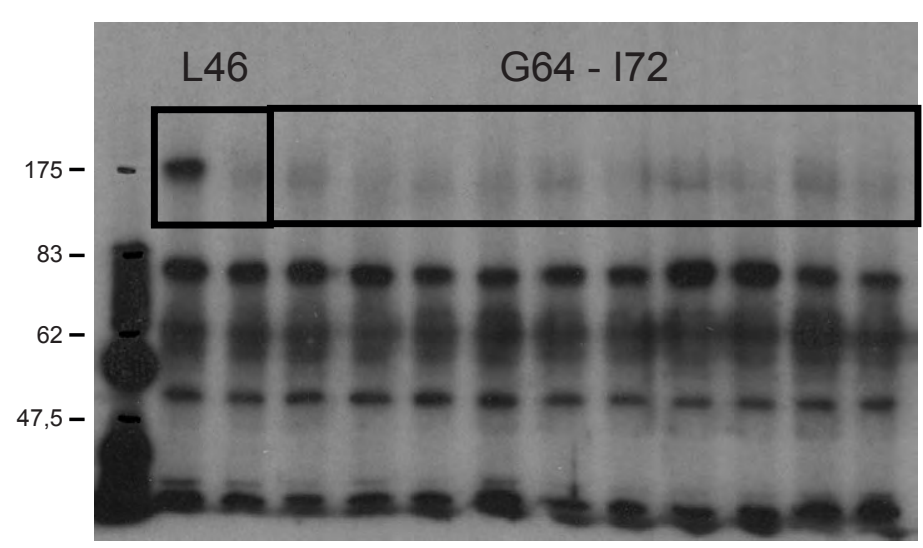

a-Hrd3

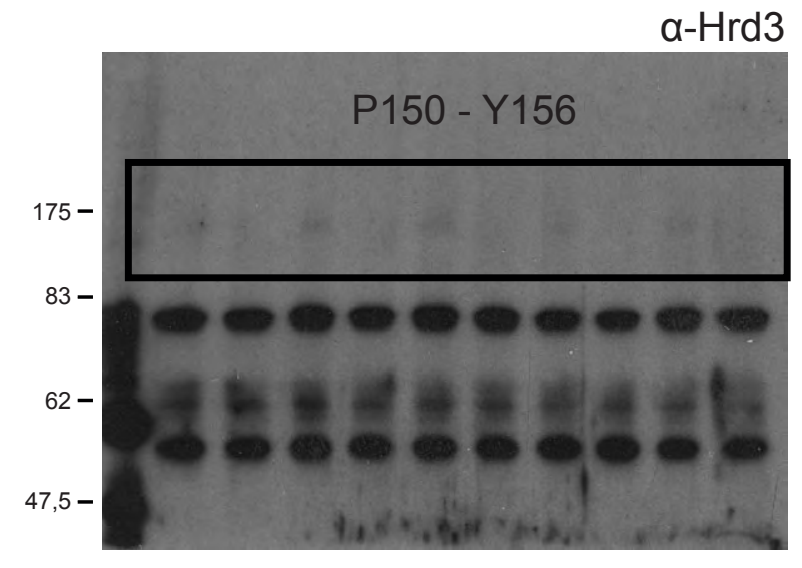

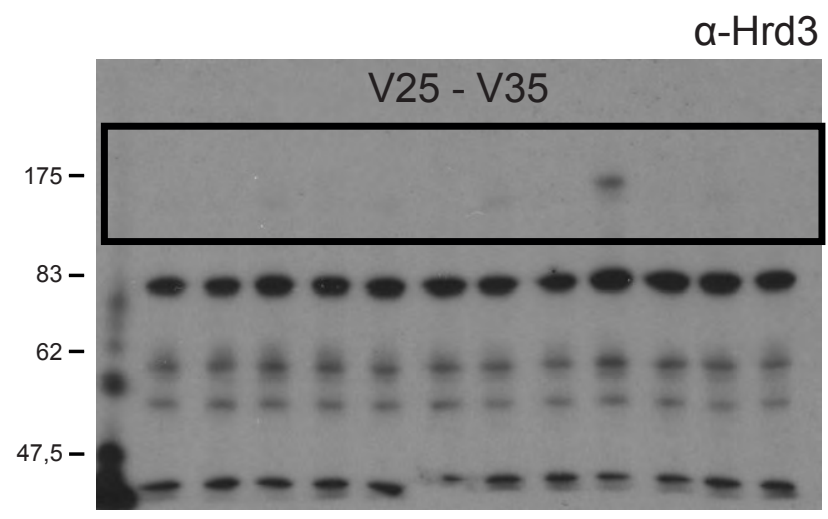

$\alpha-\mathrm{Hrd3}$

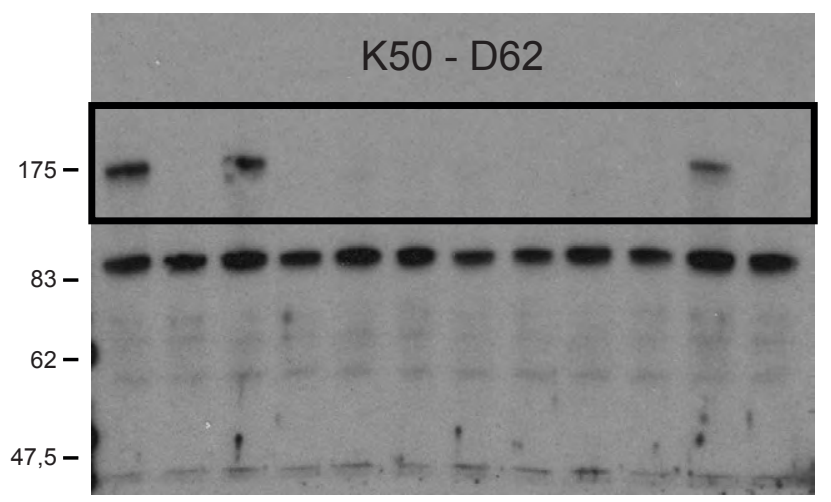

$\alpha-\mathrm{Hrd} 3$

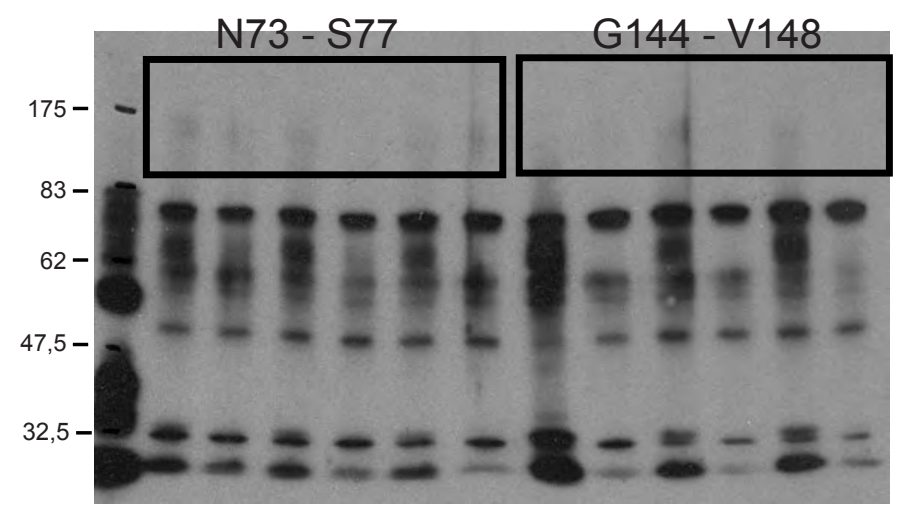

$\alpha-\mathrm{Hrd3}$

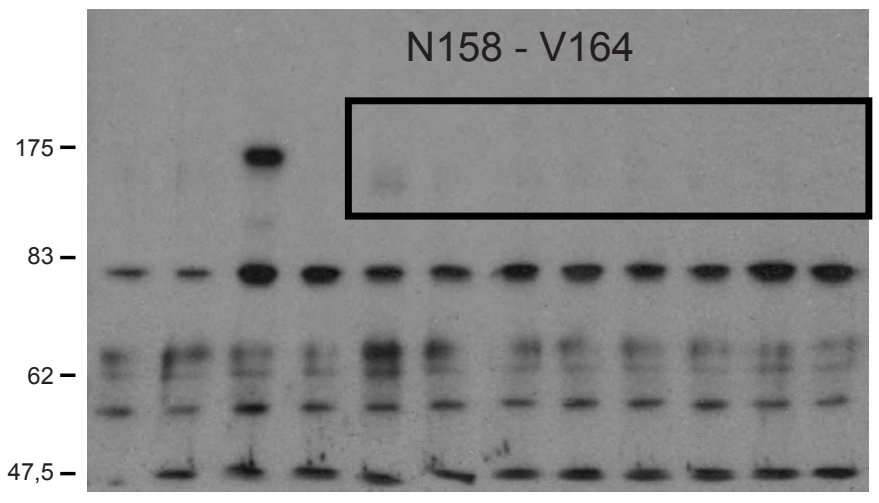


Supplementary Figure-6 (Jarosch) continued

Fig. 3
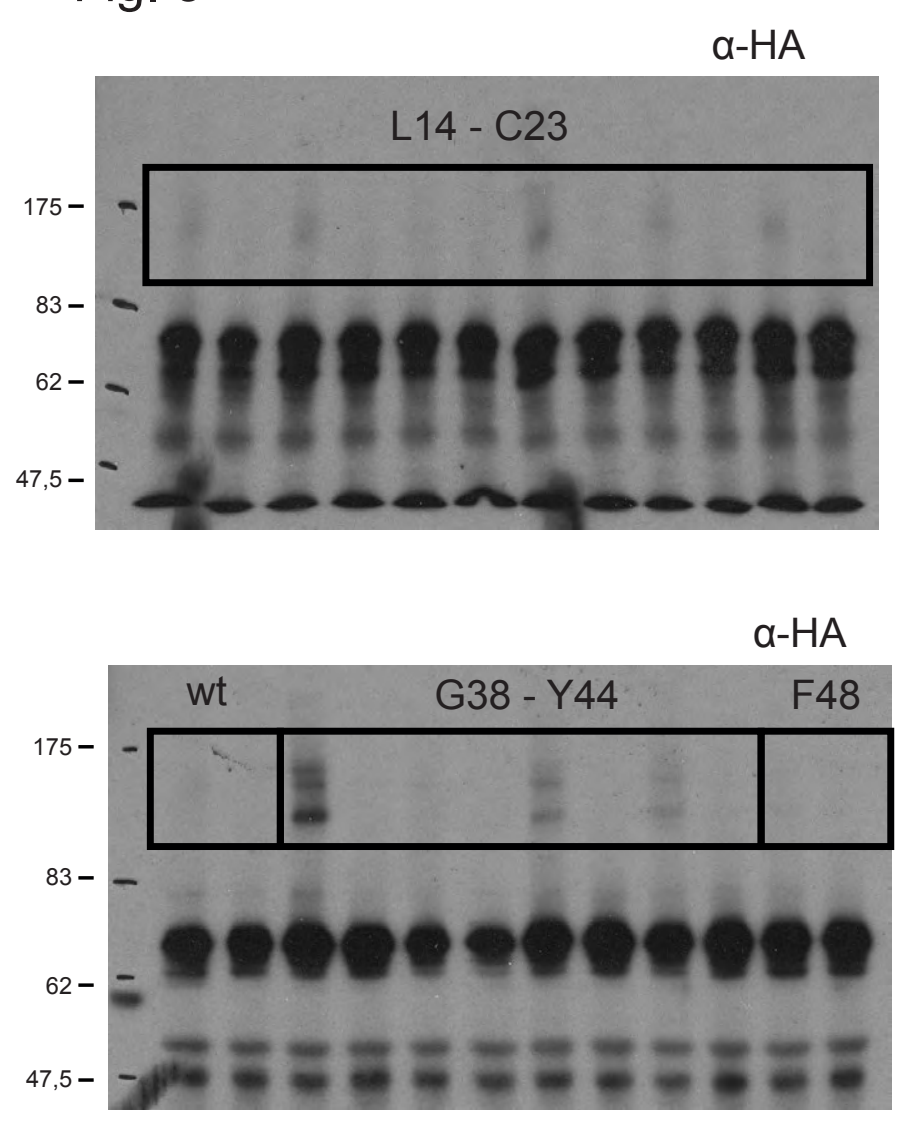

a-HA

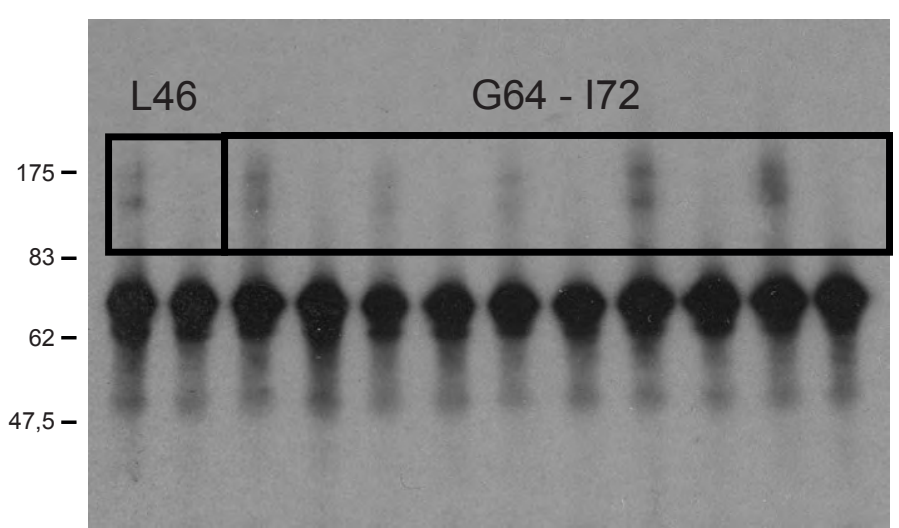

$\alpha-\mathrm{HA}$

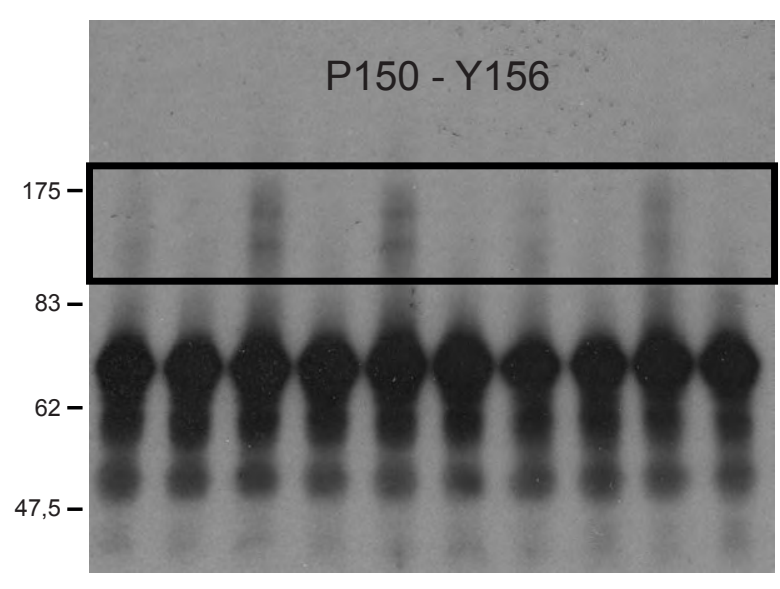

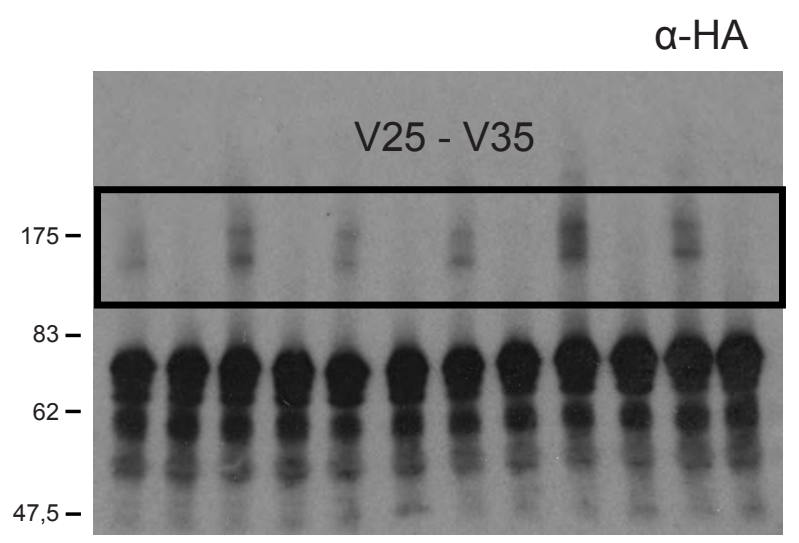

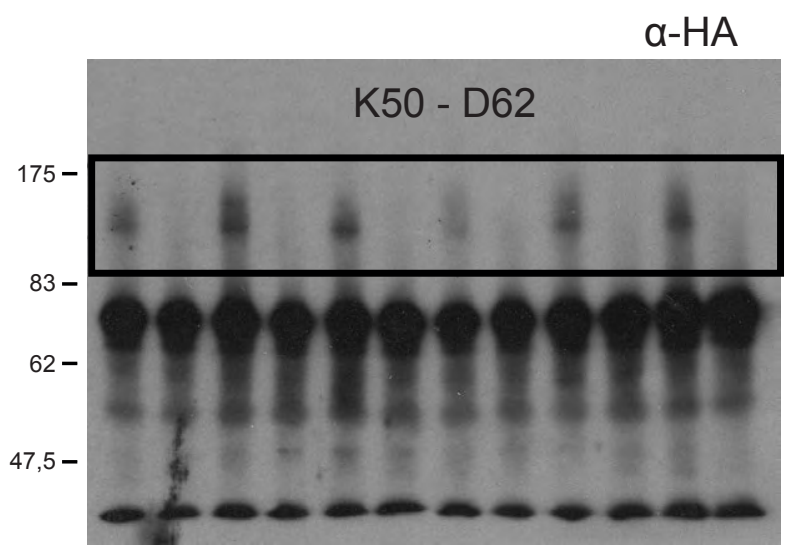

$\alpha-H A$
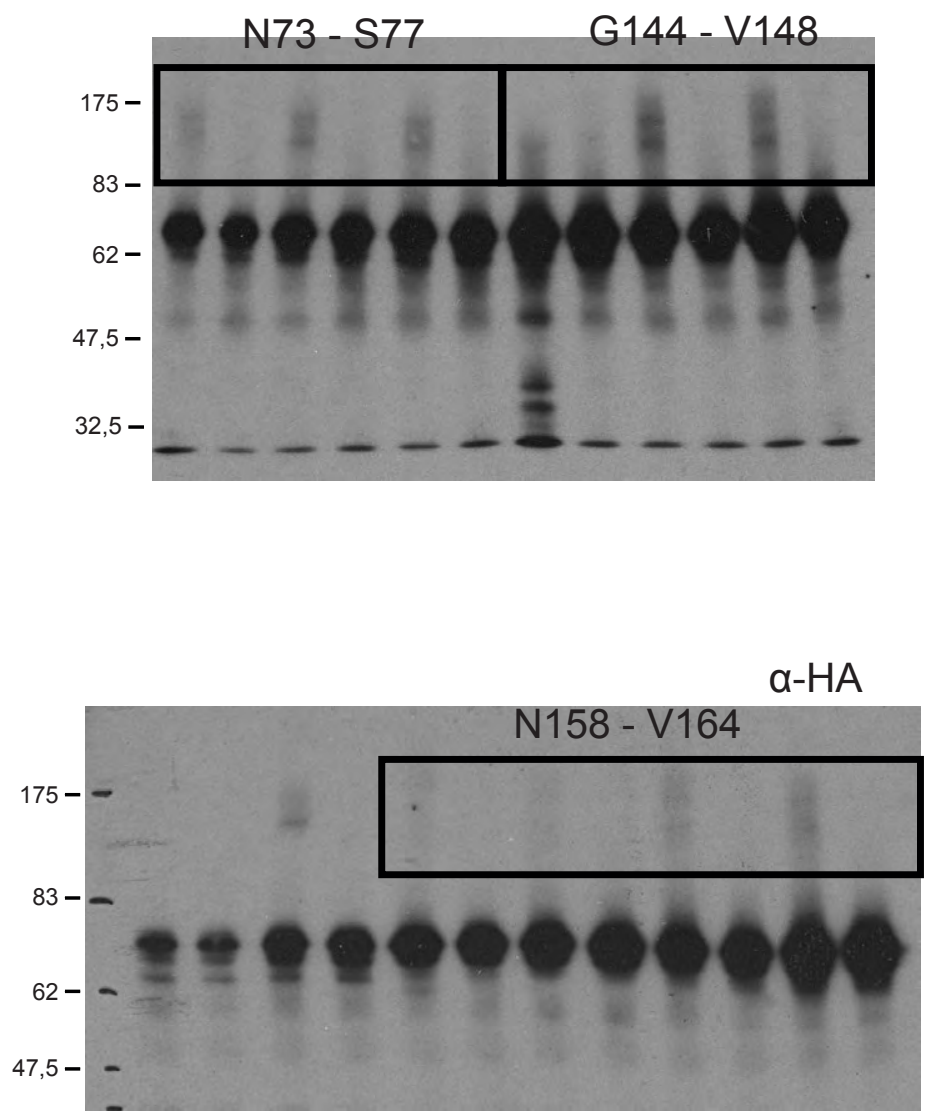
Supplementary Figure-6 (Jarosch) continued

Fig. 3
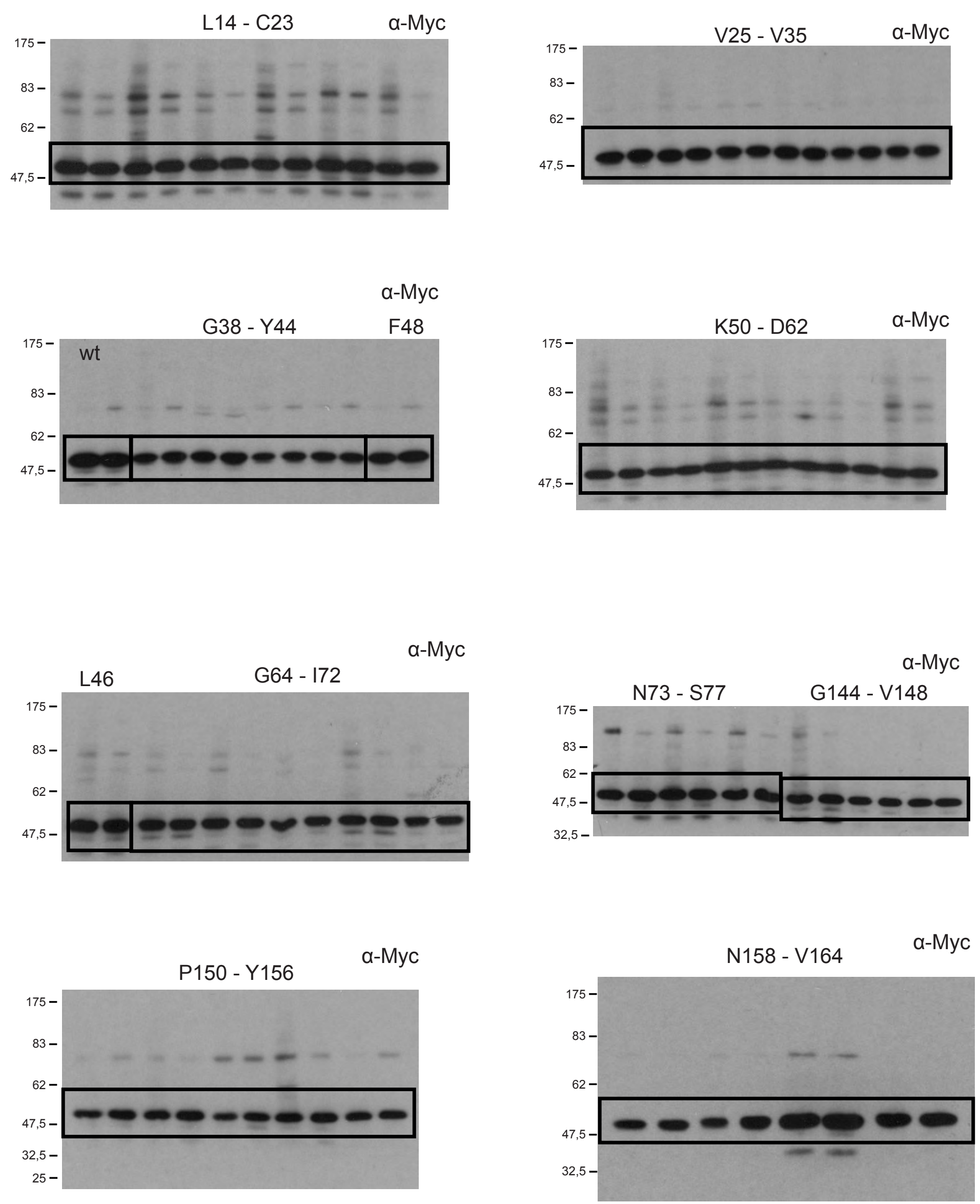
Supplementary Figure-6 (Jarosch) continued

Fig. $4 \mathrm{~b}$

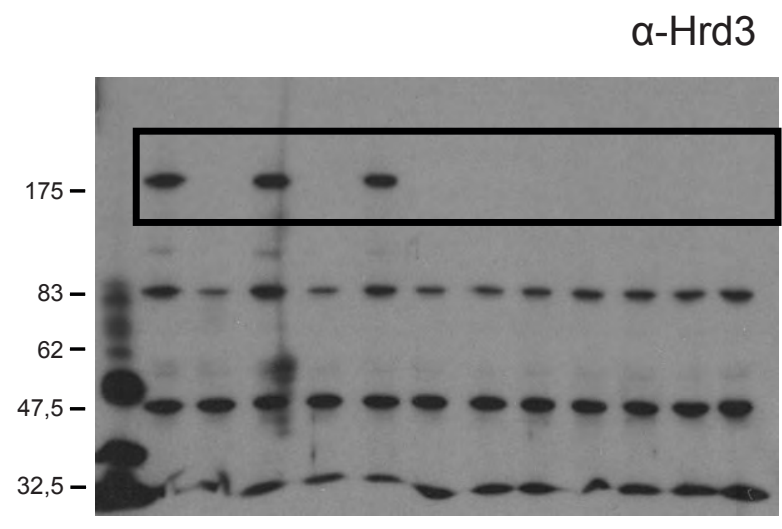

a-Hrd3
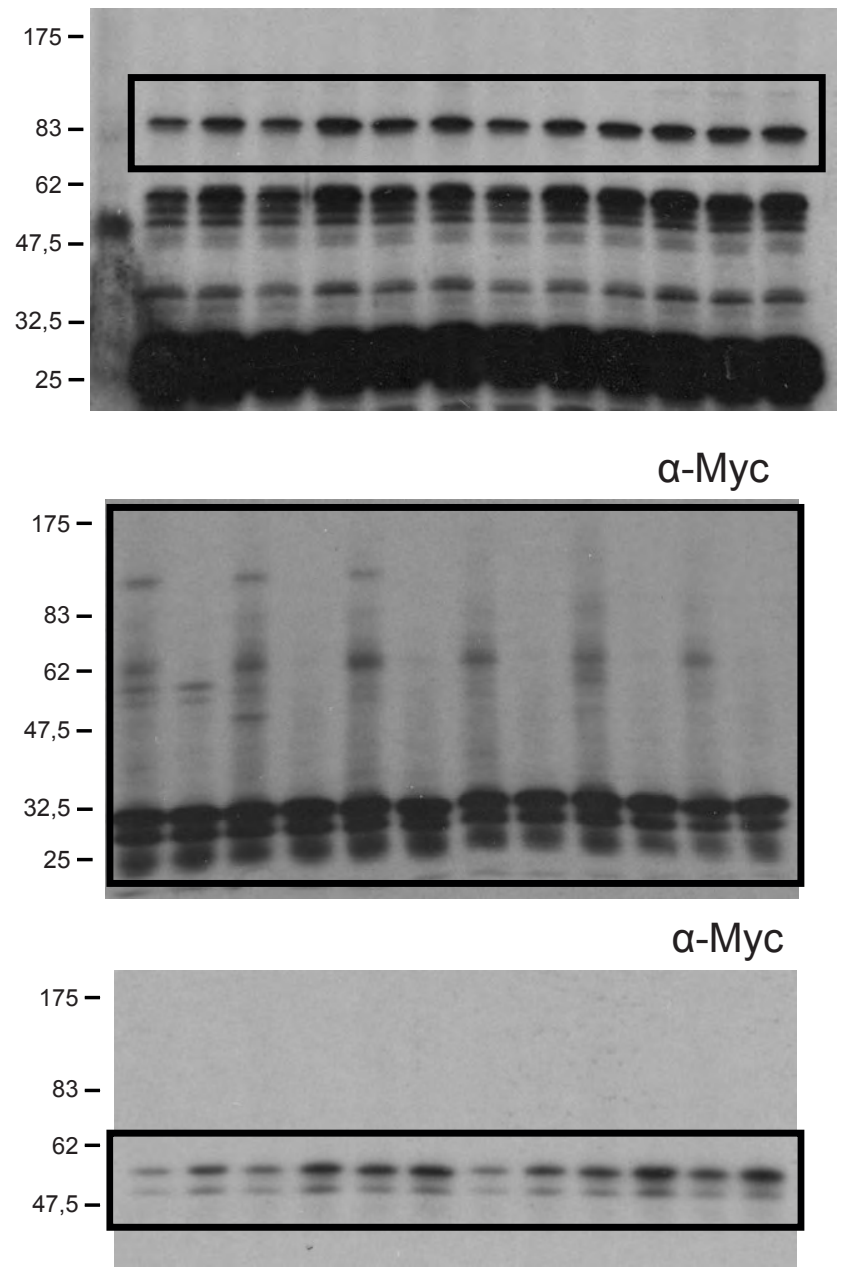

Fig. 4c

$\alpha-H r d 3$

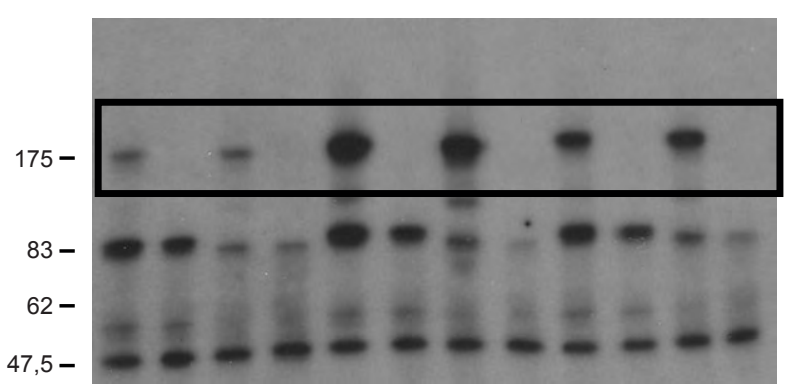

$\alpha-\mathrm{Hrd3}$
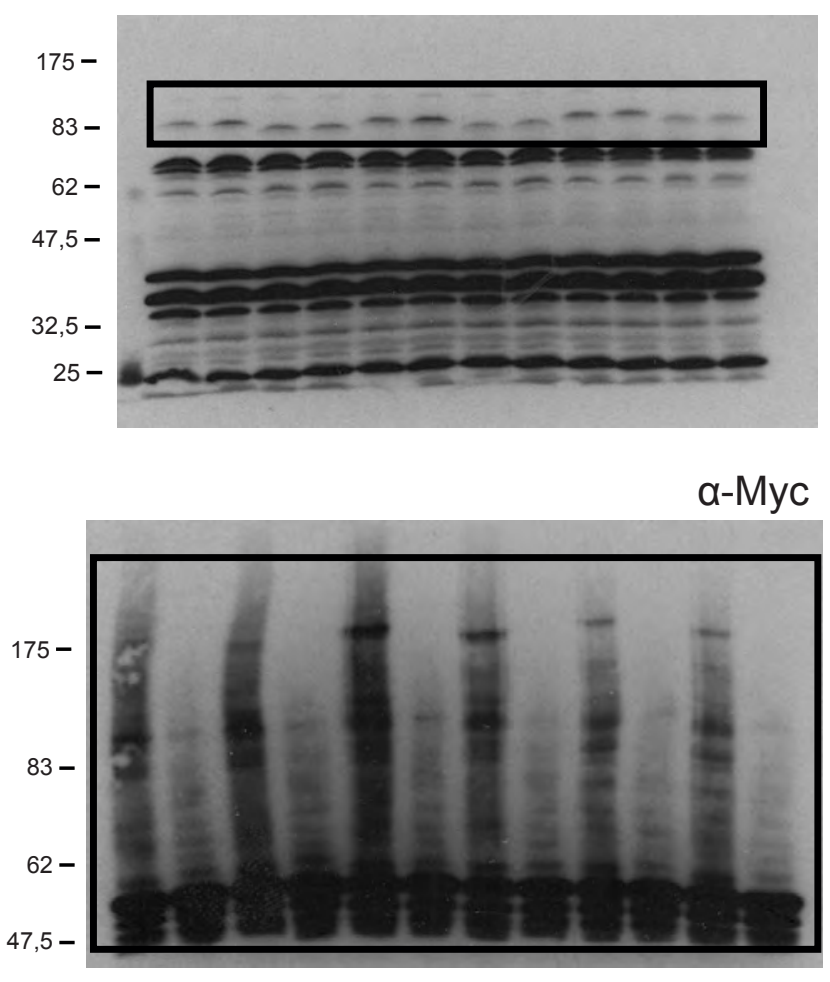

a-Myc

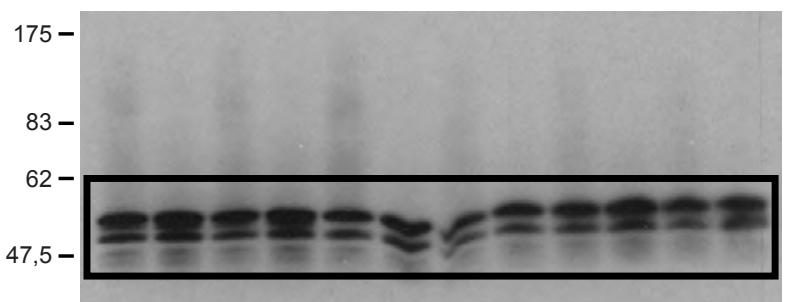


Supplementary Figure-6 (Jarosch) continued

Fig. 5a
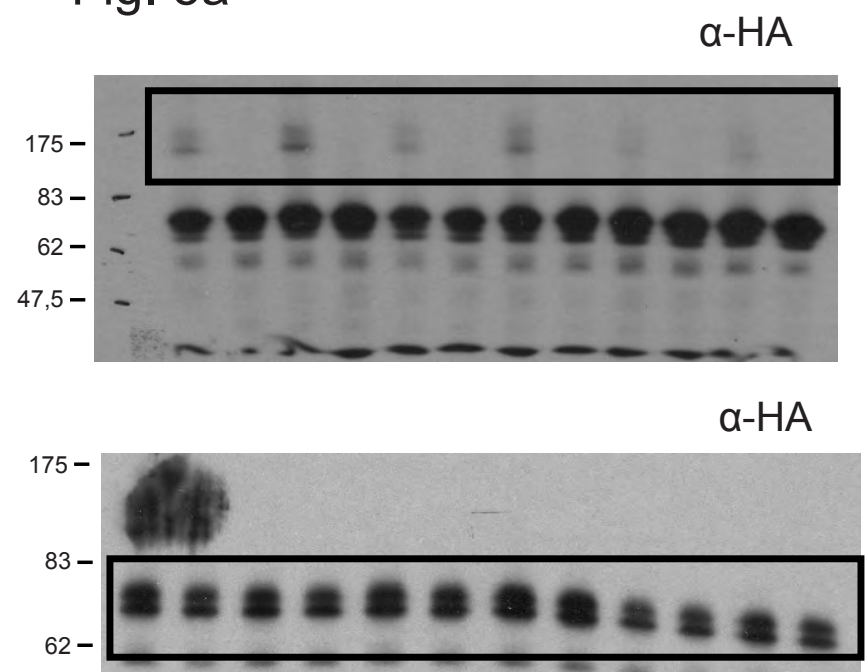

$47,5-$

$32,5-$

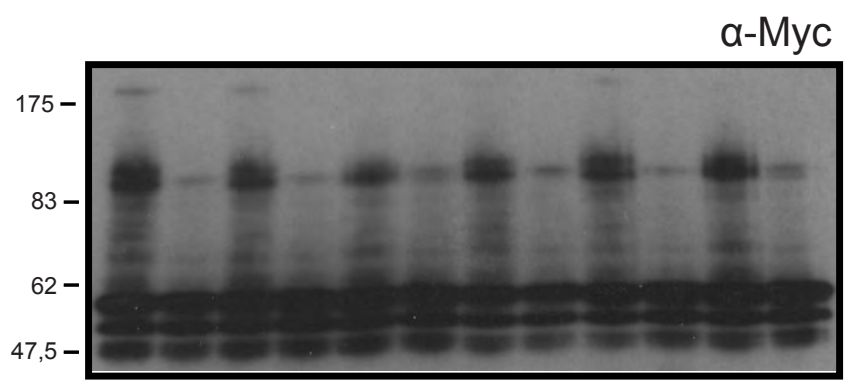

a-Myc

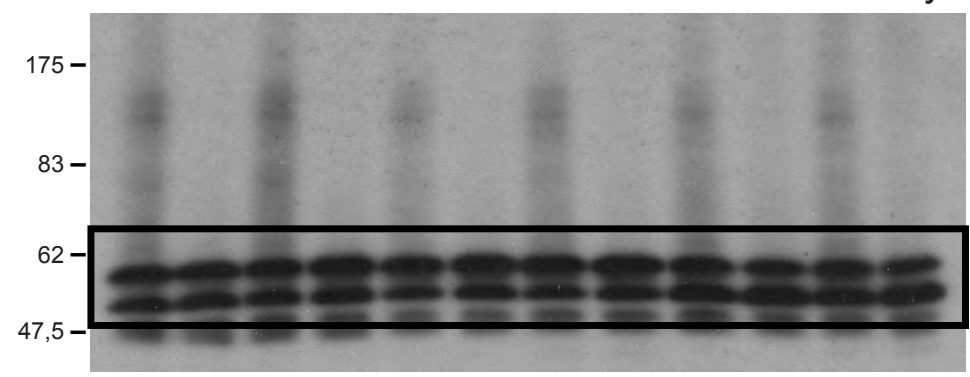

Fig. 5d
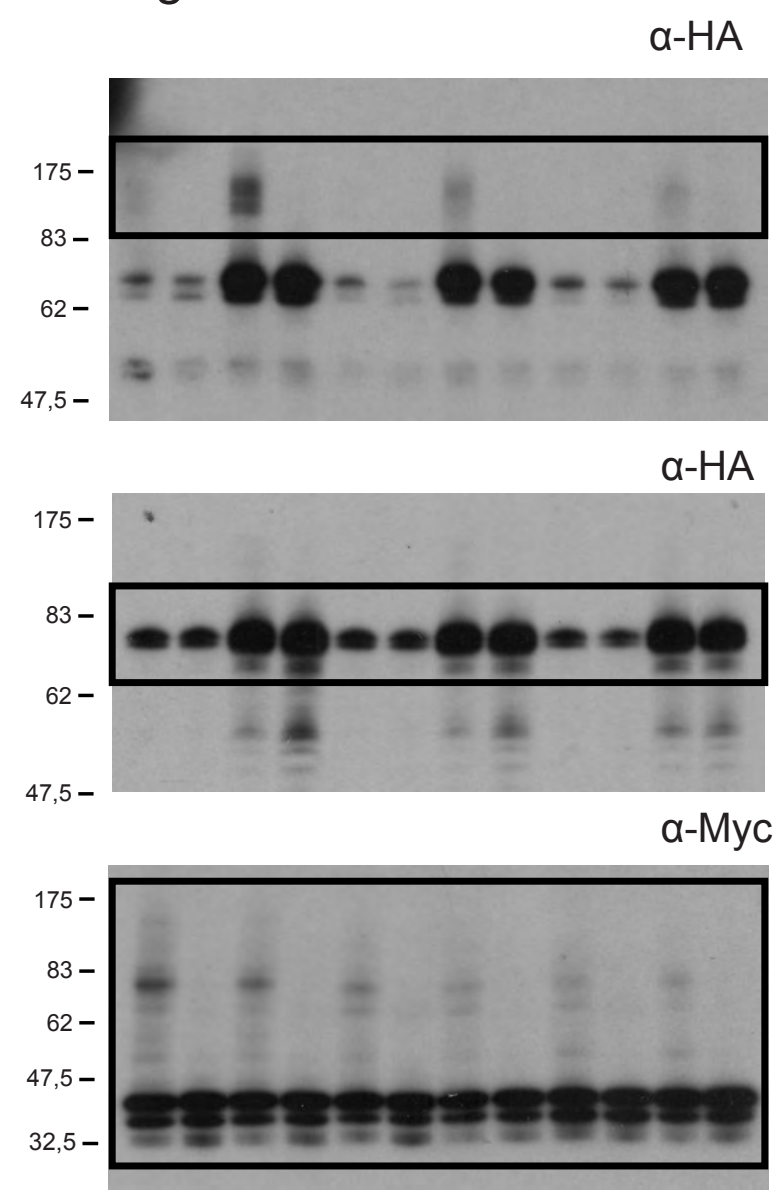

a-Myc

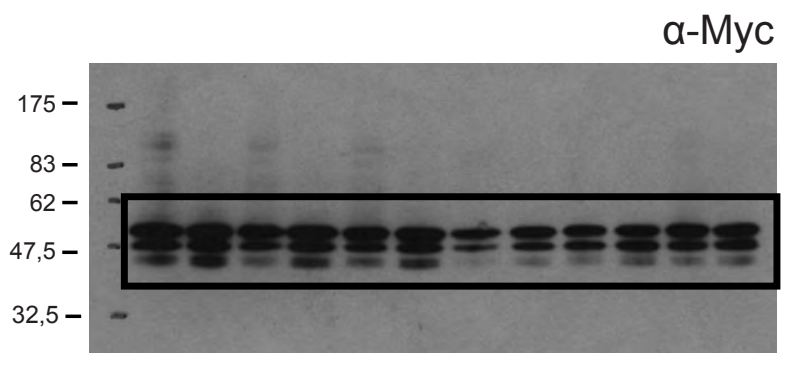


Supplementary Figure-6 (Jarosch) continued

Fig. $5 e$

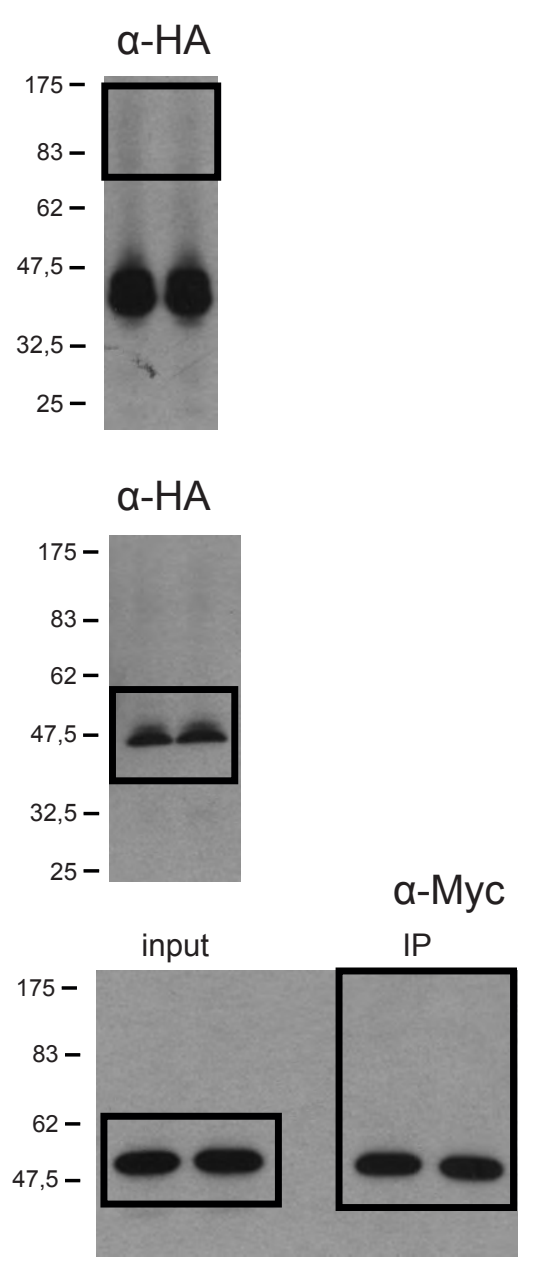

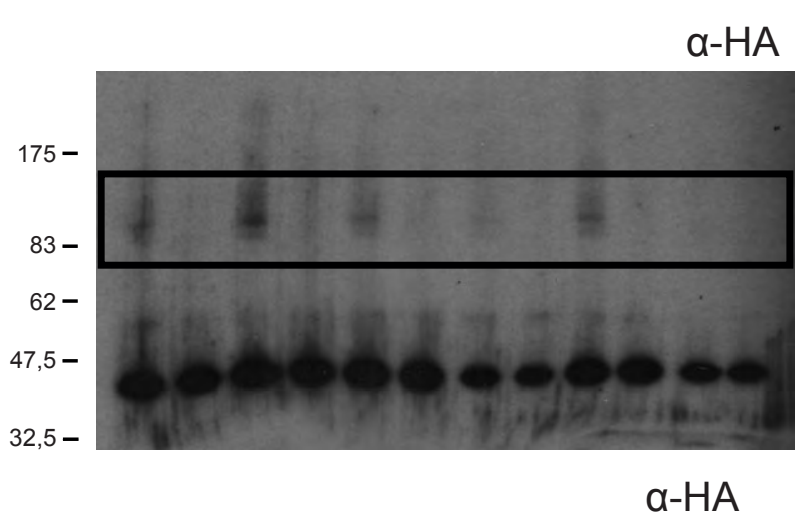
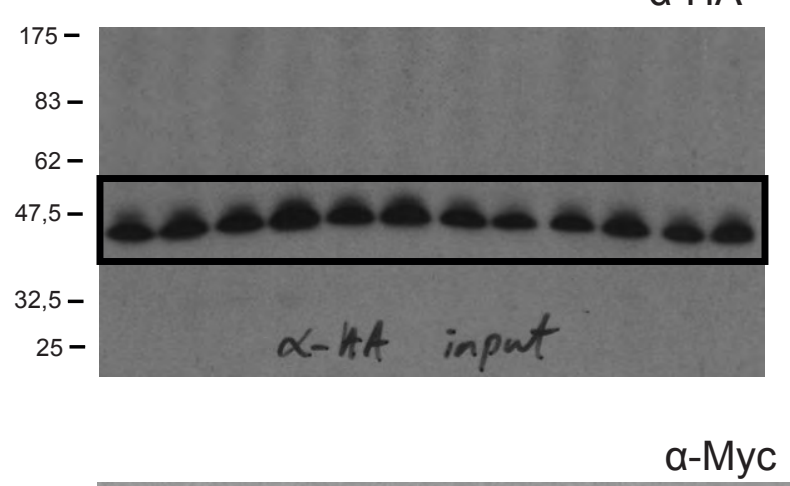

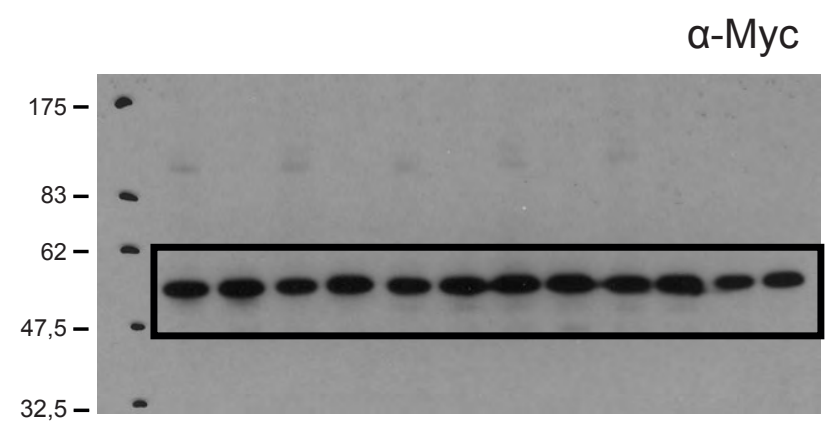


Fig. 6a
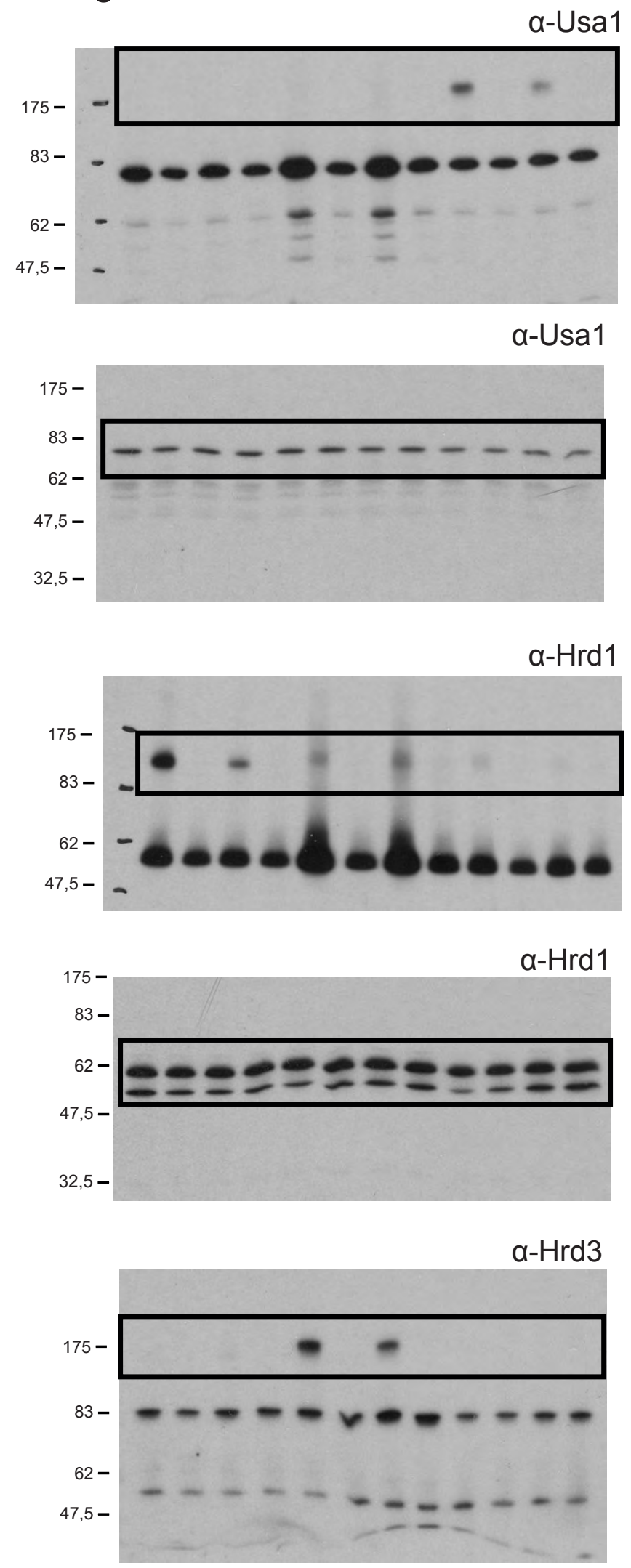

a-Hrd3

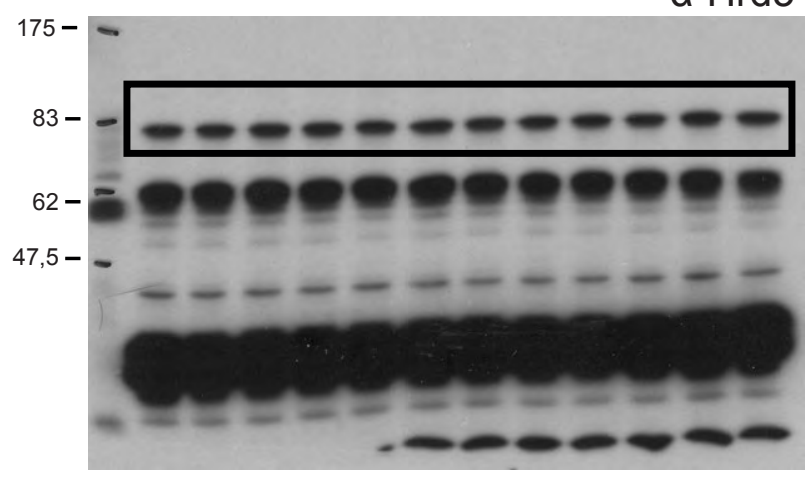

$\alpha-H A$

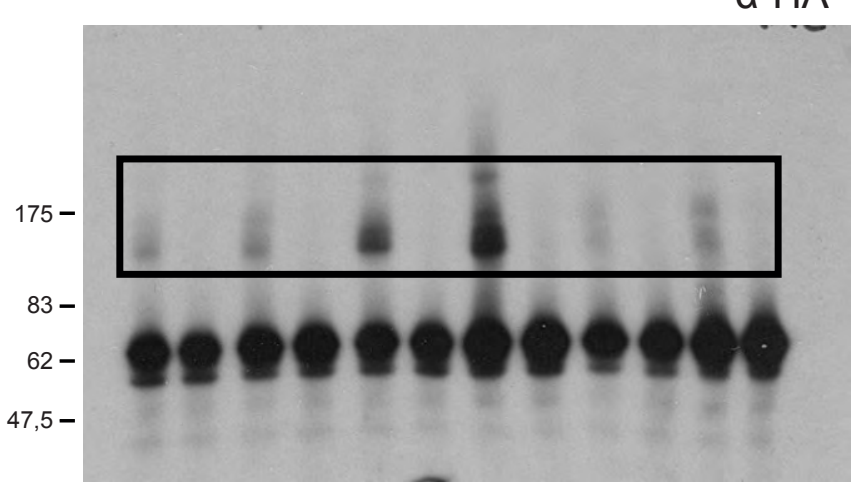

$\alpha-H A$
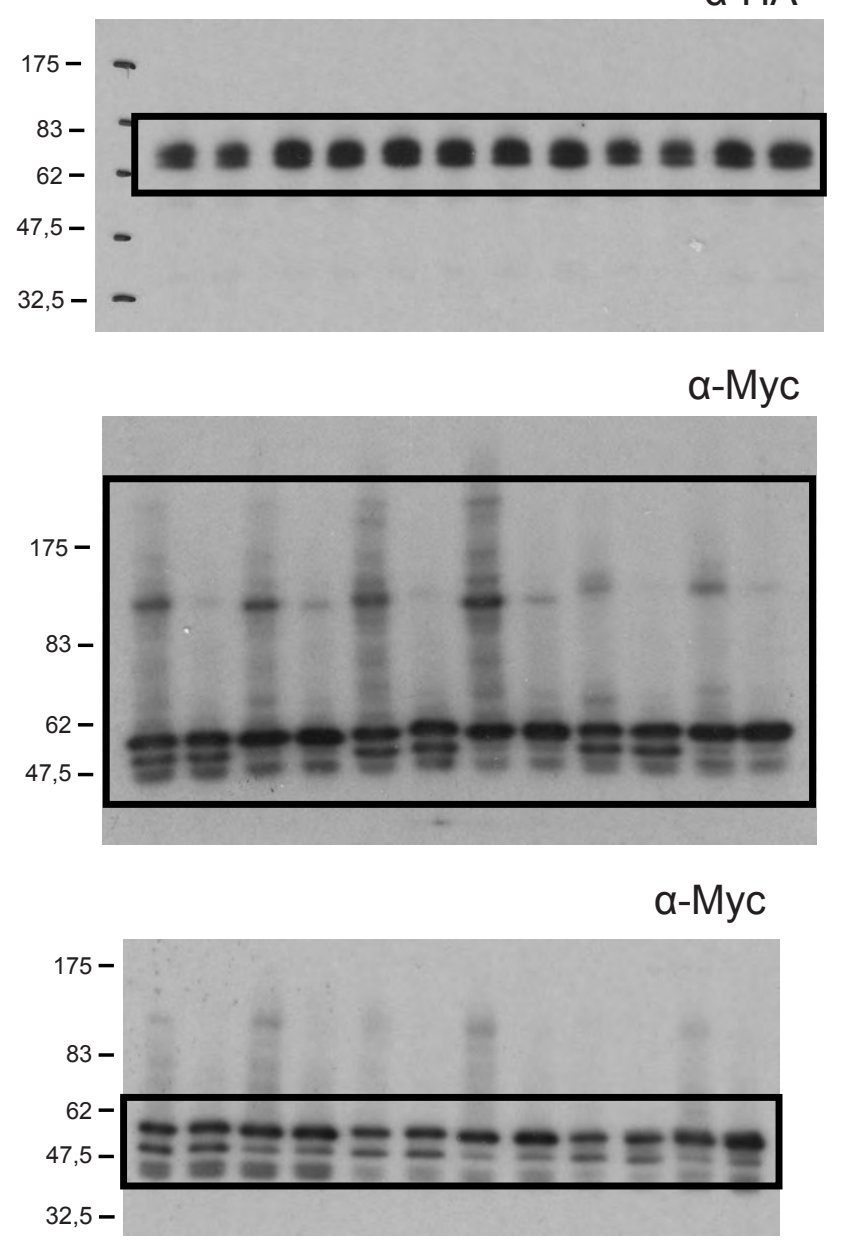
Supplementary Figure-6 (Jarosch) continued

Fig. $6 \mathrm{c}$
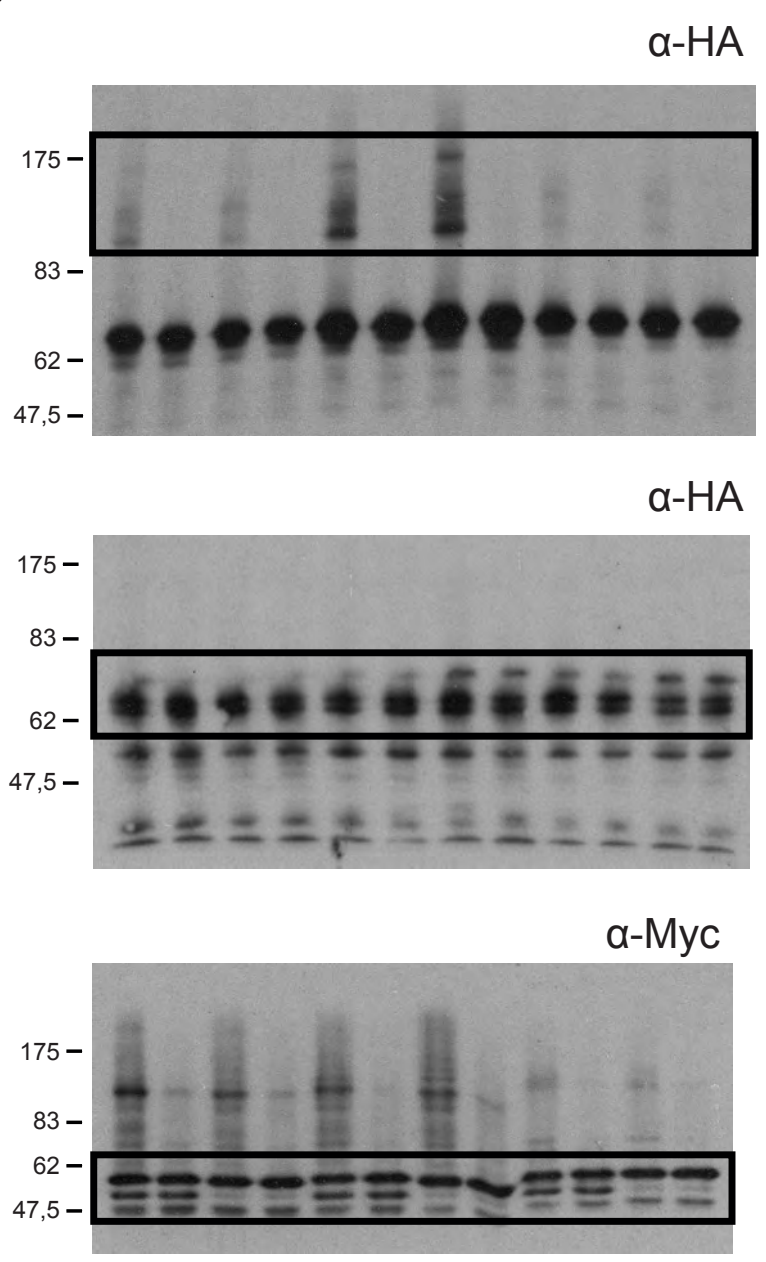

a-Myc

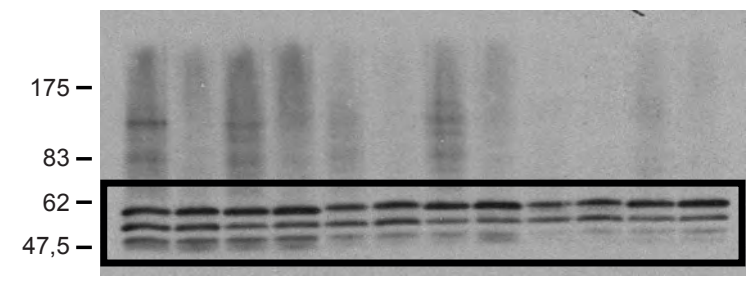


Supplementary Table 1 (Jarosch)

\begin{tabular}{|c|c|c|c|c|}
\hline Amino acid & $\begin{array}{c}\text { Der1 } \\
\text { stability }\end{array}$ & $\begin{array}{c}\mathrm{CPY}^{*} \\
\text { degradation }\end{array}$ & $\begin{array}{c}\text { Usa1 } \\
\text { interaction }\end{array}$ & $\begin{array}{c}\text { Der1 } \\
\text { oligomerisation }\end{array}$ \\
\hline T16A & + & + & n.d. & n.d. \\
\hline R17L & + & - & + & + \\
\hline C23L & + & - & + & + \\
\hline P37A & + & + & n.d. & n.d. \\
\hline G38W & + & + & n.d. & n.d. \\
\hline D45A & + & + & n.d. & n.d. \\
\hline L46A & + & + & n.d. & n.d. \\
\hline K49A & + & + & n.d. & n.d. \\
\hline K50A & + & + & n.d. & n.d. \\
\hline Q52A & + & + & n.d. & n.d. \\
\hline R55A & + & + & n.d. & n.d. \\
\hline Y58L & + & + & n.d. & n.d. \\
\hline S59L & -- & -- & n.d. & n.d. \\
\hline S70A & + & + & n.d. & n.d. \\
\hline N73L & + & - & + & + \\
\hline S77L & + & + & n.d. & n.d. \\
\hline $\begin{array}{l}\text { LEN84- } \\
\text { 86AAA }\end{array}$ & -- & -- & + & + \\
\hline R91A & + & + & n.d. & n.d. \\
\hline Y128A & + & + & n.d. & n.d. \\
\hline P154A & + & + & n.d. & n.d. \\
\hline GH179/180LA & -- & -- & -- & -- \\
\hline YY183/184AA & - & - & - & - \\
\hline DD186/187AA & + & + & + & + \\
\hline P201A & + & + & + & + \\
\hline
\end{tabular}




\section{Supplementary Table 2 (Jarosch)}

\begin{tabular}{|c|c|c|}
\hline Yeast strain & Genotype & Reference \\
\hline DF5 & $\begin{array}{l}\operatorname{trp} 1-1(\mathrm{am}) / \operatorname{trp} 1-1(\mathrm{am}), \text { his3-4200/ his3- } \triangle 200, \text { ura3-52/ ura3-52, lys2-801/lys2-801, leu2-3, -112/leu2-3, - } \\
\text { 112, MATa/a }\end{array}$ & 1 \\
\hline YBM70 & $\Delta h r d 3:: L E U 2$, prc1-1, trp1-1 (am), his3- $\triangle 200$, ura3-52, lys2-801, leu2-3, -112, MATa & 2 \\
\hline YJU32 & Ader1::HIS3, prc1-1, trp1-1 (am), his3- $\Delta 200$, ura3-52, lys2-801, leu2-3, -112, MATa & 3 \\
\hline YJU37 & Ahrd1::TRP1, prc1-1, trp1-1 (am), his3- $\Delta 200$, ura3-52, lys2-801, leu2-3, -112, MATa & 3 \\
\hline YMM010 & $\Delta h r d 1:: L E U 2, \Delta d o a 10:: H I S 3, \Delta u s a 1:: K a n M X 6$, prc1-1, MAT n.d. & this study \\
\hline YMM012 & Adoa10::HIS3, $\Delta$ usa1::KanMX6, prc1-1, trp1-1 (am), his3- $\triangle 200$, ura3-52, lys2-801, leu2-3, -112, MAT n.d. & this study \\
\hline YMM060 & $\begin{array}{l}\text { usa1-3xMyc:KanMX6, } \Delta d e r 1:: H I S 3, \Delta u b c 7:: L E U 2, \text { prc1-1, trp1-1 (am), his3- } \Delta 200, \text { ura3-52, lys2-801, leu2- } \\
\text { 3, -112, MAT n.d. }\end{array}$ & this study \\
\hline YMM111 & der1 R17L N73L, prc1-1, trp1-1 (am), his3-4200, ura3-52, lys2-801, leu2-3, -112, MATa & this study \\
\hline YMM112 & der1 R17L C23L, prc1-1, trp1-1 (am), his3- $\Delta 200$, ura3-52, lys2-801, leu2-3, -112, MATa & this study \\
\hline YMM118 & der1 R17L C23L-13xMyc:HIS3, prc1-1, trp1-1 (am), his3-4200, ura3-52, lys2-801, leu2-3, -112, MATa & this study \\
\hline YMM119 & der1 R17L N73L-13xMyc:HIS3, prc1-1, trp1-1 (am), his3-4200, ura3-52, lys2-801, leu2-3, -112, MATa & this study \\
\hline YMM132 & usa1-3xMyc:KanMX6, der1 N73L, prc1-1, trp1-1 (am), his3- 4200, ura3-52, lys2-801, leu2-3, -112, MATa & this study \\
\hline YMM133 & $\begin{array}{l}\text { usa1-3xMyc:KanMX6, der1 R17L C23L, prc1-1, trp1-1 (am), his3-4200, ura3-52, lys2-801, leu2-3, -112, } \\
\text { MATa }\end{array}$ & this study \\
\hline YMM134 & $\begin{array}{l}\text { usa1-3xMyc:KanMX6, der1 R17L N73L, prc1-1, trp1-1 (am), his3-4200, ura3-52, lys2-801, leu2-3, -112, } \\
\text { MATa }\end{array}$ & this study \\
\hline YMM138 & prc1-1-13xMyc:TRP1, der1 R17L C23L, trp1-1 (am), his3- 4200, ura3-52, lys2-801, leu2-3, -112, MATa & this study \\
\hline YMM139 & prc1-1-13xMyc:TRP1, der1 R17L N73L, trp1-1 (am), his3- 4200, ura3-52, lys2-801, leu2-3, -112, MATa & this study \\
\hline YMM140 & 6xMyc-hmg2:URA3, der1 N73L, prc1-1, trp1-1 (am), his3-4200, ura3-52, lys2-801, leu2-3, -112, MATa & this study \\
\hline YMM141 & $\begin{array}{l}\text { 6xmyc-hmg2:URA3, der1 R17L C23L, prc1-1, trp1-1 (am), his3- } \Delta 200, \text { ura3-52, lys2-801, leu2-3, -112, } \\
\text { MATa }\end{array}$ & this study \\
\hline YMM142 & $\begin{array}{l}\text { 6xMyc-hmg2:URA3, der1 R17L N73L, prc1-1, trp1-1 (am), his3- } \Delta 200, \text { ura3-52, lys2-801, leu2-3, -112, } \\
\text { MATa }\end{array}$ & this study \\
\hline YMM143 & Apep4::URA3, der1 N73L, prc1-1, trp1-1 (am), his3-4200, ura3-52, lys2-801, leu2-3, -112, MATa & this study \\
\hline YMM144 & $\Delta p e p 4:: U R A 3, \operatorname{der1} R 17 L$ C23L, prc1-1, trp1-1 (am), his3- $\Delta 200$, ura3-52, lys2-801, leu2-3, -112, MATa & this study \\
\hline YMM145 & Apep4::URA3, der1 R17L N73L, prc1-1, trp1-1 (am), his3-4200, ura3-52, lys2-801, leu2-3, -112, MATa & this study \\
\hline YMM156 & der1 N73L, prc1-1, trp1-1 (am), his3-4200, ura3-52, lys2-801, leu2-3, -112, MATa & this study \\
\hline YMM157 & ubc7 C89S, $\Delta$ der1::HIS3, prc1-1, trp1-1 (am), his3-4200, ura3-52, lys2-801, leu2-3, -112, MATa & this study \\
\hline YMM158 & $\begin{array}{l}\text { ubc7 C89S, } \triangle \text { der1::HIS3, prc1-1-3xHA:KanMX6, trp1-1 (am), his3- } \Delta 200, \text { ura3-52, lys2-801, leu2-3, -112, } \\
\text { MATa }\end{array}$ & this study \\
\hline YMM179 & der1-13xMyc:TRP1, Lusa1::HIS3, trp1-1 (am), his3-4200, ura3-52, lys2-801, leu2-3, -112, MAT n.d. & this study \\
\hline YMM187 & Dder1::HIS3, $\Delta$ hrd3::LEU2, prc1-1, trp1-1 (am), his3-4200, ura3-52, lys2-801, leu2-3, -112, MATa & this study \\
\hline YMM190 & $\begin{array}{l}\text { ubc7 C89S, } \triangle \text { der1::HIS3, } \triangle \text { hrd3::TRP1, prc1-1-3xHA:KanMX6, trp1-1 (am), his3- } \Delta 200, \text { ura3-52, lys2-801, } \\
\text { leu2-3, -112, MATa }\end{array}$ & this study \\
\hline YMM191 & $\begin{array}{l}\triangle \text { der1::HIS3, ubc7 C89S, } \triangle \text { usa1::TRP1, prc1-1, trp1-1 (am), his3- } 4200, \text { ura3-52, lys2-801, leu2-3, -112, } \\
\text { MAT n.d. }\end{array}$ & this study \\
\hline YMM199 & 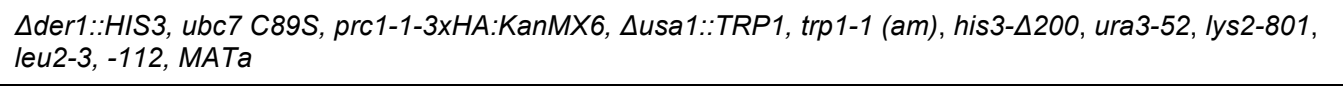 & this study \\
\hline YMM200 & $\begin{array}{l}\text { hrd1-3xHA:HIS3, ubc7 C89S, } \triangle \text { der1::TRP1, prc1-1, trp1-1 (am), his3- } \Delta 200, \text { ura3-52, lys2-801, leu2-3, - } \\
\text { 112, MAT n.d. }\end{array}$ & this study \\
\hline YMM201 & $\begin{array}{l}\text { hrd1-3xHA:HIS3, ubc7 C89S, } \Delta \text { hrd3::LEU2, prc1-1, trp1-1 (am), his3- } \Delta 200, \text { ura3-52, lys2-801, leu2-3, - } \\
\text { 112, MAT n.d. }\end{array}$ & this study \\
\hline YMM202 & $\begin{array}{l}\text { hrd1-3xHA:HIS3, ubc7 C89S, } \triangle d e r 1:: T R P 1, \Delta h r d 3:: L E U 2, \text { prc1-1, trp1-1 (am), his3- } \triangle 200, \text { ura3-52, lys2- } \\
\text { 801, leu2-3, -112, MAT n.d. }\end{array}$ & this study \\
\hline YMM203 & der1 N73L-13xMyc:TRP1, prc1-1, trp1-1 (am), his3-4200, ura3-52, lys2-801, leu2-3, -112, MATa & this study \\
\hline YMM204 & ubc7 C89S, $\Delta$ pep4::KanMX6, prc1-1, trp1-1 (am), his3-4200, ura3-52, lys2-801, leu2-3, -112, MATa & this study \\
\hline YRG184 & $\Delta$ der1::HIS3, hrd3 (1-769):KanMX6, prc1-1, trp1-1 (am), his3-4200, ura3-52, lys2-801, leu2-3, -112, MATa & 4 \\
\hline
\end{tabular}




\begin{tabular}{|c|c|c|}
\hline YSH029 & Ausa1::TRP1, prc1-1, trp1-1 (am), his3- $\triangle 200$, ura3-52, lys2-801, leu2-3, -112, MATa & 5 \\
\hline YSH061 & $\begin{array}{l}\text { der1-13xMyc:TRP1, } \Delta \text { usa1::KanMX6, prc1-1, trp1-1 (am), his3- } \Delta 200, \text { ura3-52, lys2-801, leu2-3, -112, } \\
\text { MATa }\end{array}$ & 5 \\
\hline YTX140 & prc1-1, trp1-1 (am), his3-4200, ura3-52, lys2-801, leu2-3, -112, MATa & 1 \\
\hline YTX372 & der1-13хMус:KanMX6, prc1-1, trp1-1 (am), his3-4200, ura3-52, lys2-801, leu2-3, -112, MATa & 4 \\
\hline YTX378 & $\begin{array}{l}\text { der1-13xMyc:KanMX6, } \Delta h r d 1:: T R P 1, \text { prc1-1, trp1-1 (am), his3- } \Delta 200, \text { ura3-52, lys2-801, leu2-3, -112, } \\
\text { MATa }\end{array}$ & 4 \\
\hline YTX380 & hrd1-3xHA:KanMX6, ubc7 C89S, prc1-1, trp1-1 (am), his3-4200, ura3-52, lys2-801, leu2-3, -112, MATa & 4 \\
\hline YTX404 & Ader1::HIS3, prc1-1-3xHA, trp1-1 (am), his3-4200, ura3-52, lys2-801, leu2-3, -112, MAT n.d. & this study \\
\hline YTX481 & $\begin{array}{l}\text { der1-13xMyc:KanMX6, } \Delta h r d 3:: L E U 2, \text { prc1-1, trp1-1 (am), his3- } \Delta 200, \text { ura3-52, lys2-801, leu2-3, -112, } \\
\text { MATa }\end{array}$ & this study \\
\hline YTX539 & $\begin{array}{l}\text { der1-13xMyc:KanMX6, } \Delta u b x 2:: T R P 1, \operatorname{prc1} 1 \text { 1, trp1-1 (am), his3- } \Delta 200, \text { ura3-52, lys2-801, leu2-3, -112, } \\
\text { MATa }\end{array}$ & this study \\
\hline YTX625 & usa1-3хMус:KanMX6, prc1-1, trp1-1 (am), his3-4200, ura3-52, lys2-801, leu2-3, -112, MATa & 5 \\
\hline YTX645 & Ausa1::TRP1, $\Delta h r d 1:: L E U 2$, prc1-1, trp1-1 (am), his3-4200, ura3-52, lys2-801, leu2-3, -112, MATa & this study \\
\hline YTX740 & 6xMyc-hmg2:URA3, $\Delta h r d 1:: T R P 1, \operatorname{prc1-1}, \operatorname{trp1-1}(\mathrm{am})$, his3- $\triangle 200$, ura3-52, lys2-801, leu2-3, -112, MATa & 5 \\
\hline YTX742 & 6xMyc-hmg2:URA3, prc1-1, trp1-1 (am), his3-4200, ura3-52, lys2-801, leu2-3, -112, MATa & 5 \\
\hline YTX817 & prc1-1-13xMyc:TRP1, trp1-1 (am), his3-4200, ura3-52, lys2-801, leu2-3, -112, MATa & 5 \\
\hline YTX822 & prc1-1-13xMyc:TRP1, $\Delta$ der1::HIS3, trp1-1 (am), his3-4200, ura3-52, lys2-801, leu2-3, -112, MATa & 5 \\
\hline YTX836 & Apep4::URA3, prc1-1, trp1-1 (am), his3- 200 , ura3-52, lys2-801, leu2-3, -112, MATa & 5 \\
\hline YTX837 & Apep4::URA3, $\triangle$ der1::HIS3, prc1-1, trp1-1 (am), his3- $\triangle 200$, ura3-52, lys2-801, leu2-3, -112, MATa & 5 \\
\hline YTX865 & prc1-1-13xMyc:TRP1, rpt4R, trp1-1 (am), his3- $\Delta 200$, ura3-52, lys2-801, leu2-3, -112, MATa & this study \\
\hline
\end{tabular}

MAT n.d. Mating type not determined

\section{References}

1. Biederer, T., Volkwein, C. \& Sommer, T. Role of Cue1p in ubiquitination and degradation at the ER surface. Science 278, 18061809 (1997).

2. Meusser, B. \& Sommer, T. Vpu-mediated degradation of CD4 reconstituted in yeast reveals mechanistic differences to cellular ER-associated protein degradation. Mol Cell 14, 247-258 (2004).

3. Walter, J., Urban, J., Volkwein, C. \& Sommer, T. Sec61p-independent degradation of the tail-anchored ER membrane protein Ubc6p. EMBO J 20, 3124-3131 (2001).

4. Gauss, R., Sommer, T. \& Jarosch, E. The Hrd1p ligase complex forms a linchpin between ER-lumenal substrate selection and

Cdc48p recruitment. EMBO J 25, 1827-1835 (2006).

5. Horn, S.C. et al. Usa1 functions as a scaffold of the HRD-ubiquitin ligase. Mol Cell 36, $782-793$ (2009). 
Supplementary Table 3 (Jarosch)

\begin{tabular}{|c|c|c|c|}
\hline Plasmid & Plasmid backbone & Description & Reference \\
\hline pTX339 & pRS415 & $\operatorname{PrA}^{*}$ & 1 \\
\hline pGK1-pBpa & & 3SUP4-tRNACUA for pBpa incorporation & 2 \\
\hline $\mathrm{pRH} 244$ & & 6xMyc-Hmg2 for genomic integration & 3 \\
\hline pTX227 & pRS416 & Der1 -499/+728 & this study \\
\hline pTX228 & pTX227 & Der1 (R17L) & this study \\
\hline pTX230 & pTX227 & Der1 (C23L) & this study \\
\hline pTX231 & pTX227 & Der1 (N73L) & this study \\
\hline pMM001 & pRS406 & Der1 -499/+728 (N73L) & this study \\
\hline pMM012 & pTX227 & Der1 (T16A) & this study \\
\hline pMM013 & pTX227 & Der1 (S70A) & this study \\
\hline pMM014 & pTX227 & Der1 (LEN84-86AAA) & this study \\
\hline pMM015 & pTX227 & Der1 (R91A) & this study \\
\hline pMM016 & pTX227 & Der1 (Y128A) & this study \\
\hline pMM017 & pTX227 & Der1 (P154A) & this study \\
\hline pMM020 & pTX227 & Der1 (GH179LA) & this study \\
\hline pMM021 & pTX227 & Der1 (YY183AA) & this study \\
\hline pMM022 & $\mathrm{pTX} 227$ & Der1 (DD186AA) & this study \\
\hline pMM023 & pTX227 & Der1 (P201A) & this study \\
\hline pMM040 & pRS406 & Der1 -499/+728 (R17LC23LN73L) & this study \\
\hline pMM041 & pTX406 & Der1 -499/+728 (R17LN73L) & this study \\
\hline pMM043 & pTX227 & Der1 (R17LC23L) & this study \\
\hline pMM044 & pTX227 & Der1 (R17LN73L) & this study \\
\hline pMM046 & pTX227 & Der1 (K50A) & this study \\
\hline pMM047 & pTX227 & Der1 (R55A) & this study \\
\hline pMM048 & pTX227 & Der1 (Y58L) & this study \\
\hline pMM049 & pTX227 & Der1 (Q52A) & this study \\
\hline pMM052 & pTX227 & Der1 (S77L) & this study \\
\hline pMM053 & pTX227 & Der1 (S59L) & this study \\
\hline pMM054 & pTX227 & Der1 (P37A) & this study \\
\hline pMM055 & pTX227 & Der1 (G38W) & this study \\
\hline pMM056 & pTX227 & Der1 (D45A) & this study \\
\hline pMM057 & pTX227 & Der1 (L46A) & this study \\
\hline pMM058 & pTX227 & Der1 (K49A) & this study \\
\hline pMM063 & pRS425-CUP & Der1 & this study \\
\hline pMM064 & pRS416 & Der1 -500/+636-1xHA & this study \\
\hline pMM074 & pRS425-CUP & Der1 -500/+706 (R17LN73L)-13xMyc & this study \\
\hline pMM075 & pRS425-CUP & Der1 -499/+706-13xMyc & this study \\
\hline pMM076 & pRS413 & $\operatorname{PrA}^{*}-3 \times \mathrm{HA}$ & this study \\
\hline
\end{tabular}

References

1. Horn, S.C. et al. Usa1 functions as a scaffold of the HRD-ubiquitin ligase. Mol Cell 36, 782-793 (2009).

2. Chen, S., Schultz, P.G. \& Brock, A. An improved system for the generation and analysis of mutant proteins containing unnatural amino acids in Saccharomyces cerevisiae. J Mol Biol 371, 112-122 (2007).

3. Hampton, R.Y., Gardner, R.G. \& Rine, J. Role of 26S proteasome and HRD genes in the degradation of 3-hydroxy-3methylglutaryl-CoA reductase, an integral endoplasmic reticulum membrane protein. Mol Biol Cell 7, 2029-2044 (1996). 